\title{
Rhodium(III)-Catalyzed Diastereoselective Ring-Opening of 7-Azabenzonorbornadienes with Aromatic Ketoximes: Synthesis of Benzophenanthridine Derivatives
}

Varathan Vinayagam, Arumugam Mariappan, Mrinmoy Jana and Masilamani Jeganmohan*

Department of Chemistry, Indian Institute of Technology Madras, Chennai 600036,

Tamil Nadu, India

Email:mjeganmohan@iitm.ac.in

\section{Supporting Information (SI)}

Table of Contents

S2 - S3 X-Ray analysis Data

S4 - S5 Mechanistic Investigation

S6 - S75 Copies of ${ }^{1} \mathrm{H}$ and ${ }^{13} \mathrm{C}$ NMR Spectra of All Compounds. 


\section{Crystallographic Data of Compound 3ba:}

Suitable single crystals for X-ray diffraction studies were obtained from the compound synthesized in this study. Single crystals were grown in a NMR tube at room temperature using $\mathrm{CDCl}_{3}$ over a period of 3-4 weeks by slow evaporation of solvent.

X-ray data was collected with a Bruker AXS (Kappa Apex 2) CCD diffractometer equipped with graphite monochromatic Mo $(\mathrm{K} \alpha)(\lambda=0.7107 \mathrm{~A})$ radiation source. The data were collected with $100 \%$ completeness for $\Theta$ up to $25^{\circ}$. $\omega$ and $\phi$ scans were employed to collect the data. The frame width for $\omega$ for was fixed to $0.5^{\circ}$ for data collection. The crystal was solved by direct methods using Bruker SHELXS (Sheldrick, 1997). The Structure was refined using the Bruker SHELXTL (Version 6.12) software package. These data were deposited with Cambridge Crystallographic Data Center with the following numbers: CCDC 1950036.

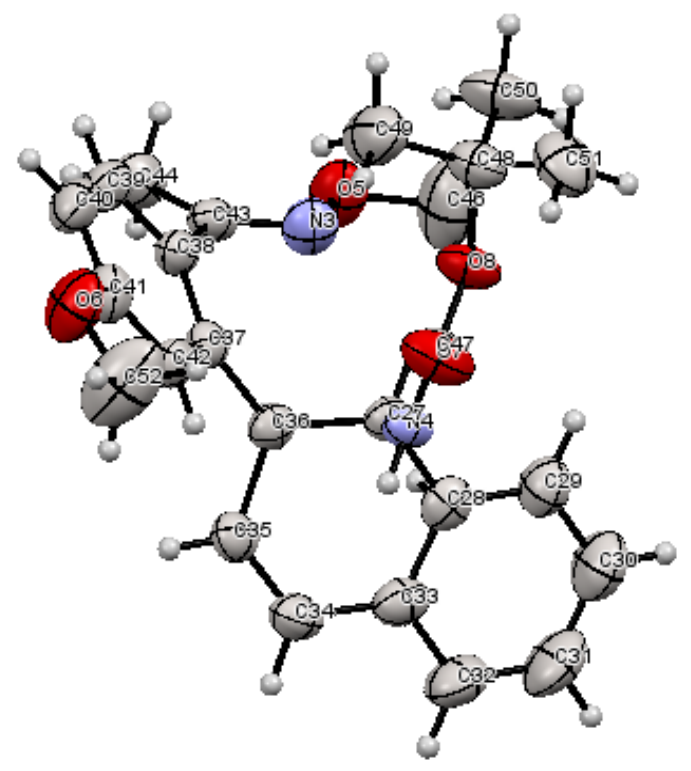

Figure S1: ORTEP representation of compound 3ba displaying thermal elliposoid at 50\% probability. 
X-Ray Analysis of Compounds 3ba (the crystal is having dimeric compound).

Identification code

Empirical formula

Formula weight

Temperature

Wavelength

Crystal system

Space group

Unit cell dimensions

Volume

Z

Density (calculated)

Absorption coefficient

$\mathrm{F}(000)$

Crystal size

Theta range for data collection

Index ranges

Reflections collected

Independent reflections

Completeness to theta $=24.292^{\circ}$

Absorption correction

Refinement method

Data / restraints / parameters

Goodness-of-fit on $\mathrm{F}^{2}$

Final R indices [I>2sigma(I)]

$\mathrm{R}$ indices (all data)

Extinction coefficient

Largest diff. peak and hole 3ba

$\mathrm{C}_{50} \mathrm{H}_{61} \mathrm{~N}_{4} \mathrm{O}_{8}$

846.02

296(2) K

$71.073 \mathrm{pm}$

Triclinic

P -1

$\mathrm{a}=1114.05(16) \mathrm{pm}$

$\alpha=89.364(5)^{\circ}$.

$\mathrm{b}=1469.54(16) \mathrm{pm}$

$\beta=79.407(4)^{\circ}$.

$\mathrm{c}=1523.2(2) \mathrm{pm}$

$\gamma=75.874(4)^{\circ}$.
$1.183 \mathrm{Mg} / \mathrm{m}^{3}$

$0.080 \mathrm{~mm}^{-1}$

906

$0.250 \times 0.220 \times 0.150 \mathrm{~mm}^{3}$

1.361 to $24.292^{\circ}$.

$-12<=\mathrm{h}<=12,-16<=\mathrm{k}<=16,-17<=\mathrm{l}<=17$

28630

$7586[\mathrm{R}($ int $)=0.0592]$

$98.3 \%$

None

Full-matrix least-squares on $\mathrm{F}^{2}$

7586 / 0 / 580

1.062

$\mathrm{R} 1=0.0609, \mathrm{wR} 2=0.1617$

$\mathrm{R} 1=0.1138, \mathrm{wR} 2=0.1996$

$0.0043(11)$

0.214 and -0.591 e. $\AA^{-3}$ 
${ }^{1} \mathrm{H}$ Spectra of Compound D-1c $\left(\mathrm{CDCl}_{3}\right.$ was used).

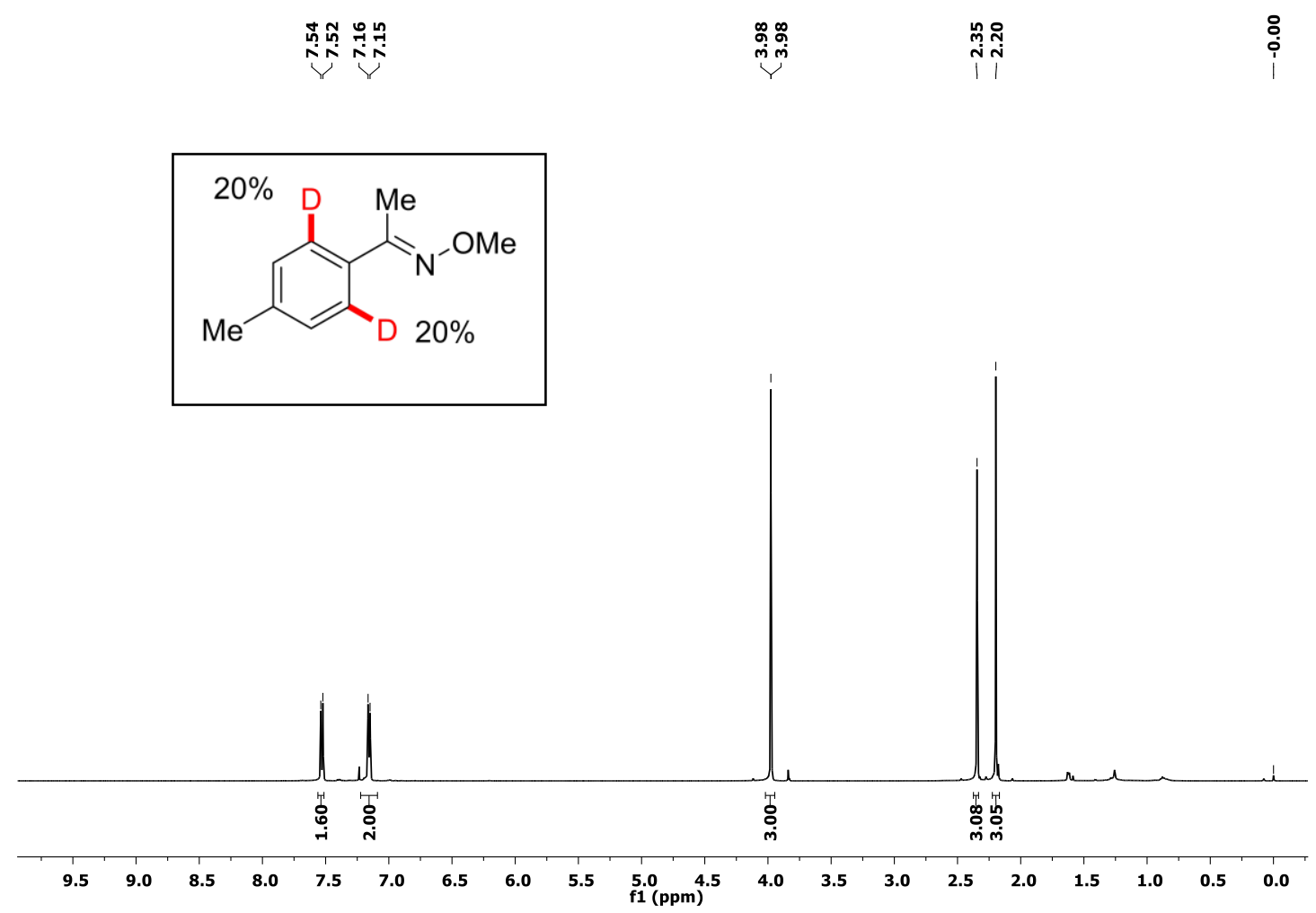

${ }^{1} \mathrm{H}$ Spectra of Compound D-3ca $\left(\mathrm{CDCl}_{3}\right.$ was used).

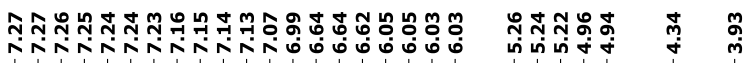

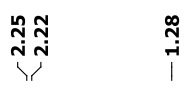

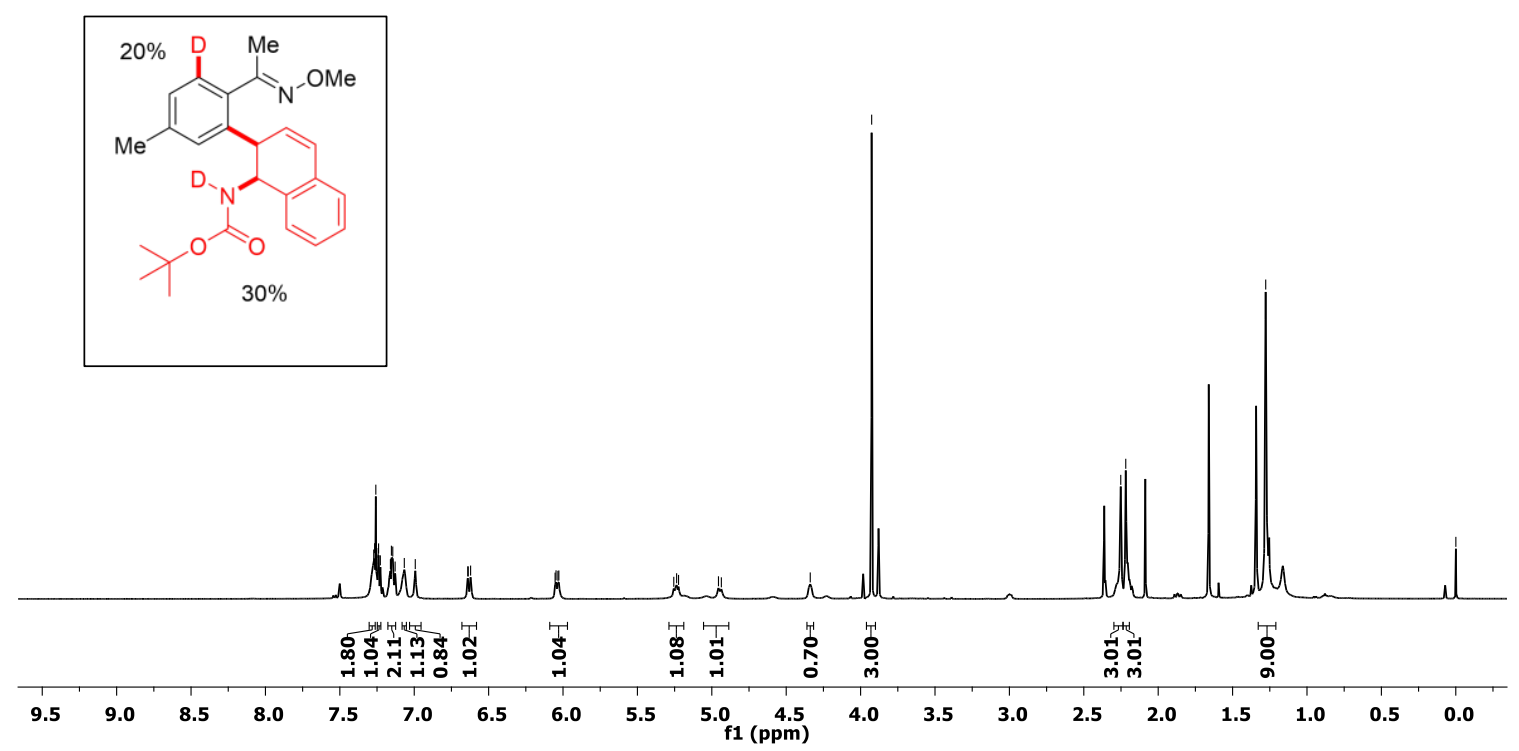


Isolation of intermediate $B$.

${ }^{1} \mathrm{H}$ and ${ }^{13} \mathrm{C}$ NMR Spectra of Compound $\mathbf{B}$

ใด้

岗

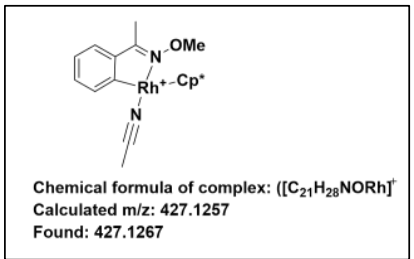

whil

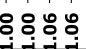

$\begin{array}{llllllllllll}9.5 & 9.0 & 8.5 & 8.0 & 7.5 & 7.0 & 6.5 & 6.0 & 5.5 & 5.0 & 4.5 & 4.0\end{array}$

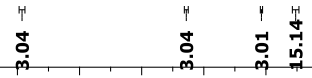

i

糸

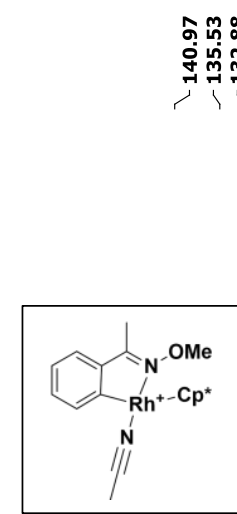

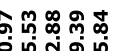

ช้ำ สู่

ํํำ

ח

웅

:

in

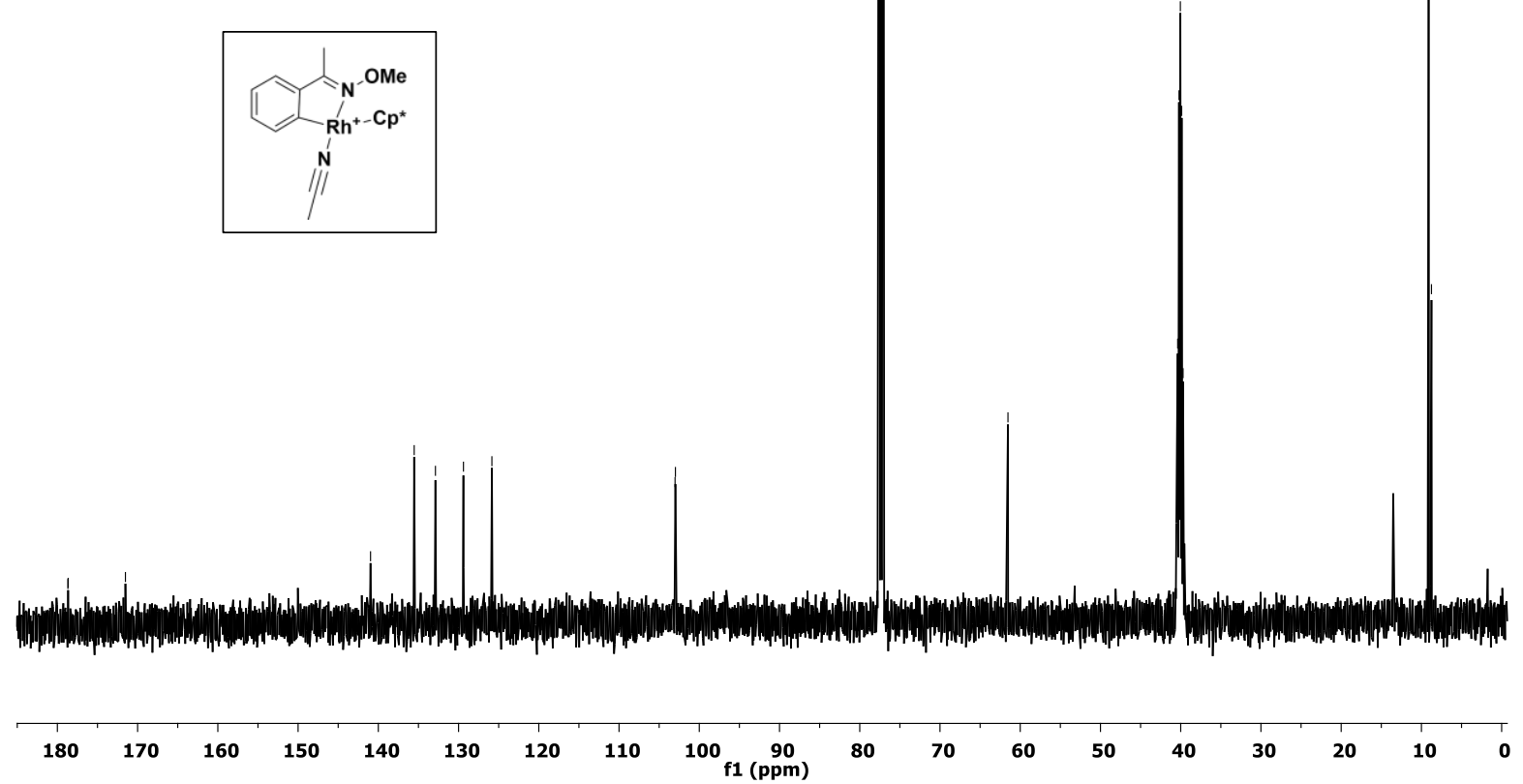


${ }^{1} \mathrm{H}$ and ${ }^{13} \mathrm{C}$ NMR Spectra of Compound 3aa.

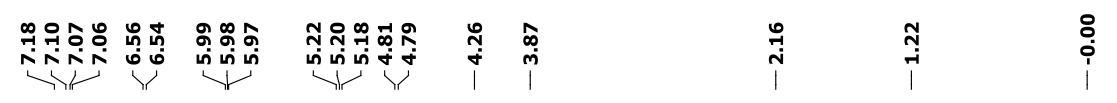

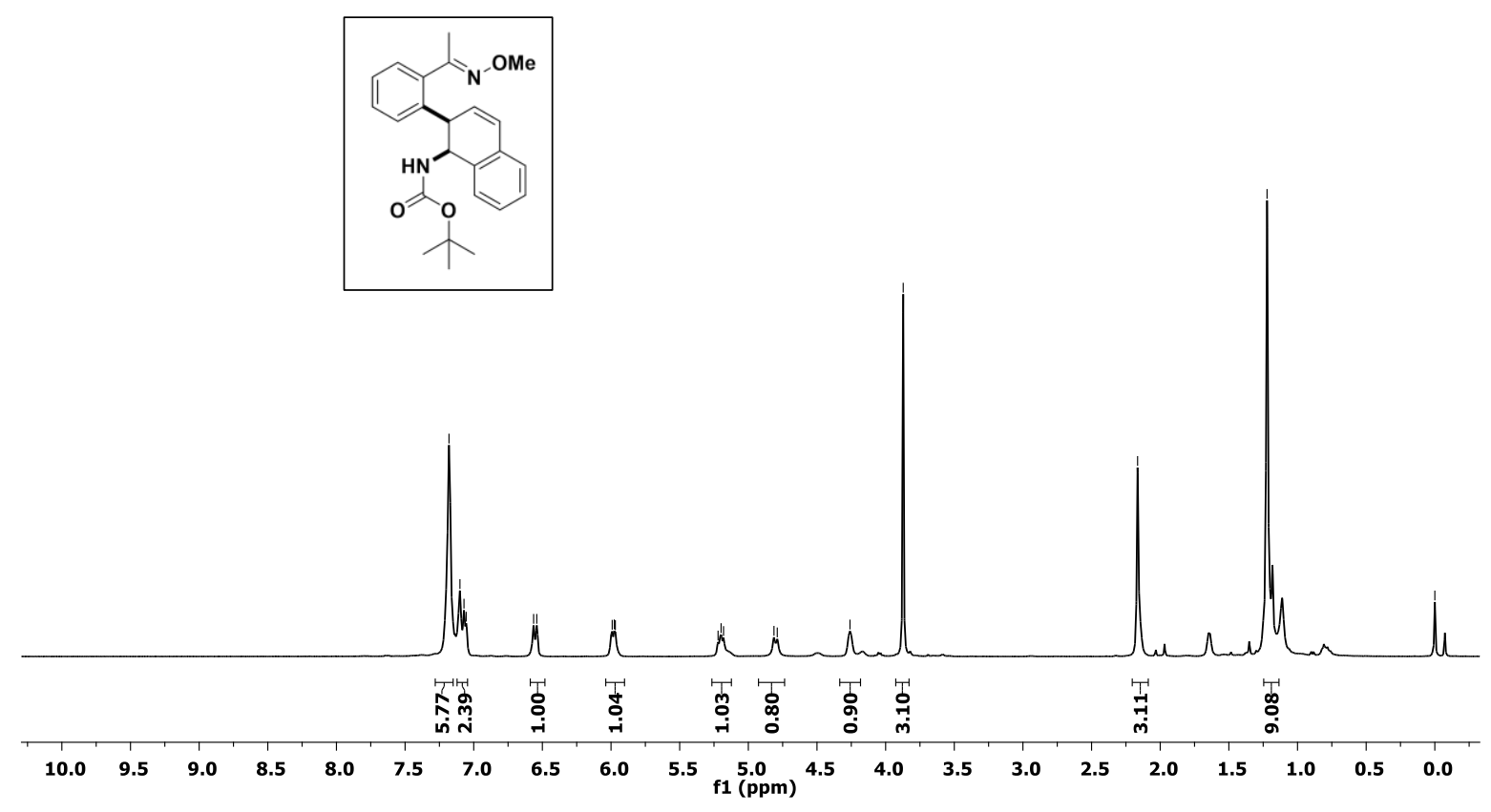

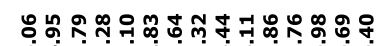

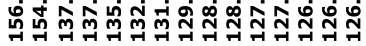
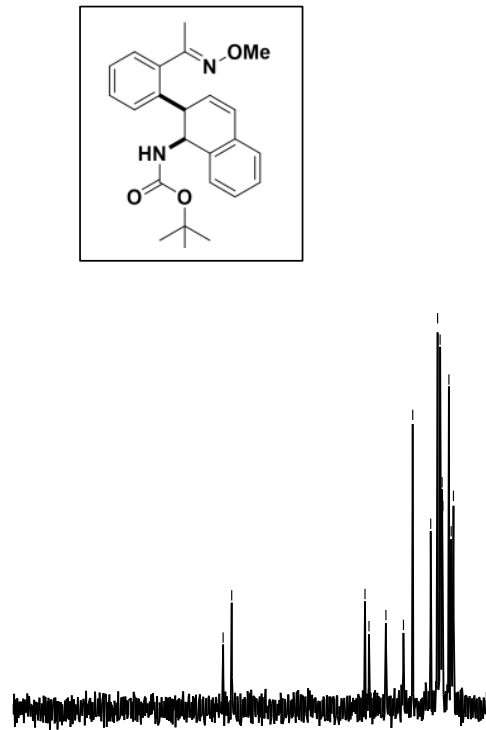

18

$\begin{array}{llllllllll}170 & 160 & 150 & 140 & 130 & 120 & 110 & 100 & 90 \begin{array}{r}80 \\ \text { f1 (ppm) }\end{array}\end{array}$

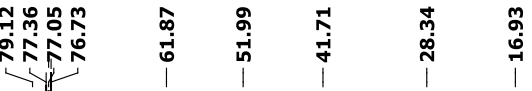


DEPT (135) NMR Spectrum of Compound 3aa.

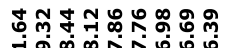

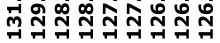

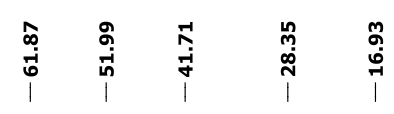
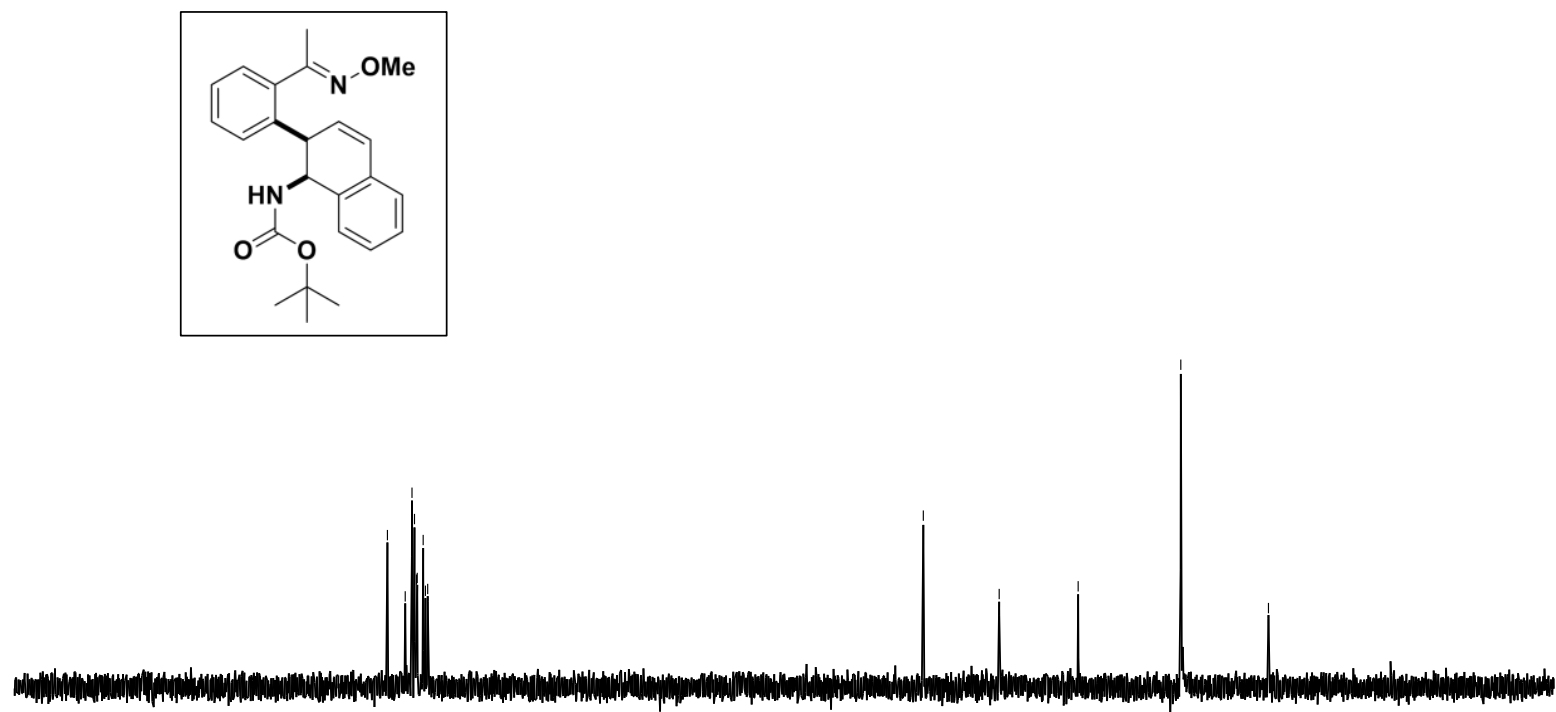

$\begin{array}{lllllllllllllllllllll}180 & 170 & 160 & 150 & 140 & 130 & 120 & 110 & 100 & 90 & \underset{f 1}{8(\mathrm{ppm})} & 70 & 60 & 50 & 40 & 30 & 20 & 10 & 0 & -10 & -2 C\end{array}$ 
${ }^{1} \mathrm{H}$ and ${ }^{13} \mathrm{C}$ NMR Spectra of Compound 3 ba.
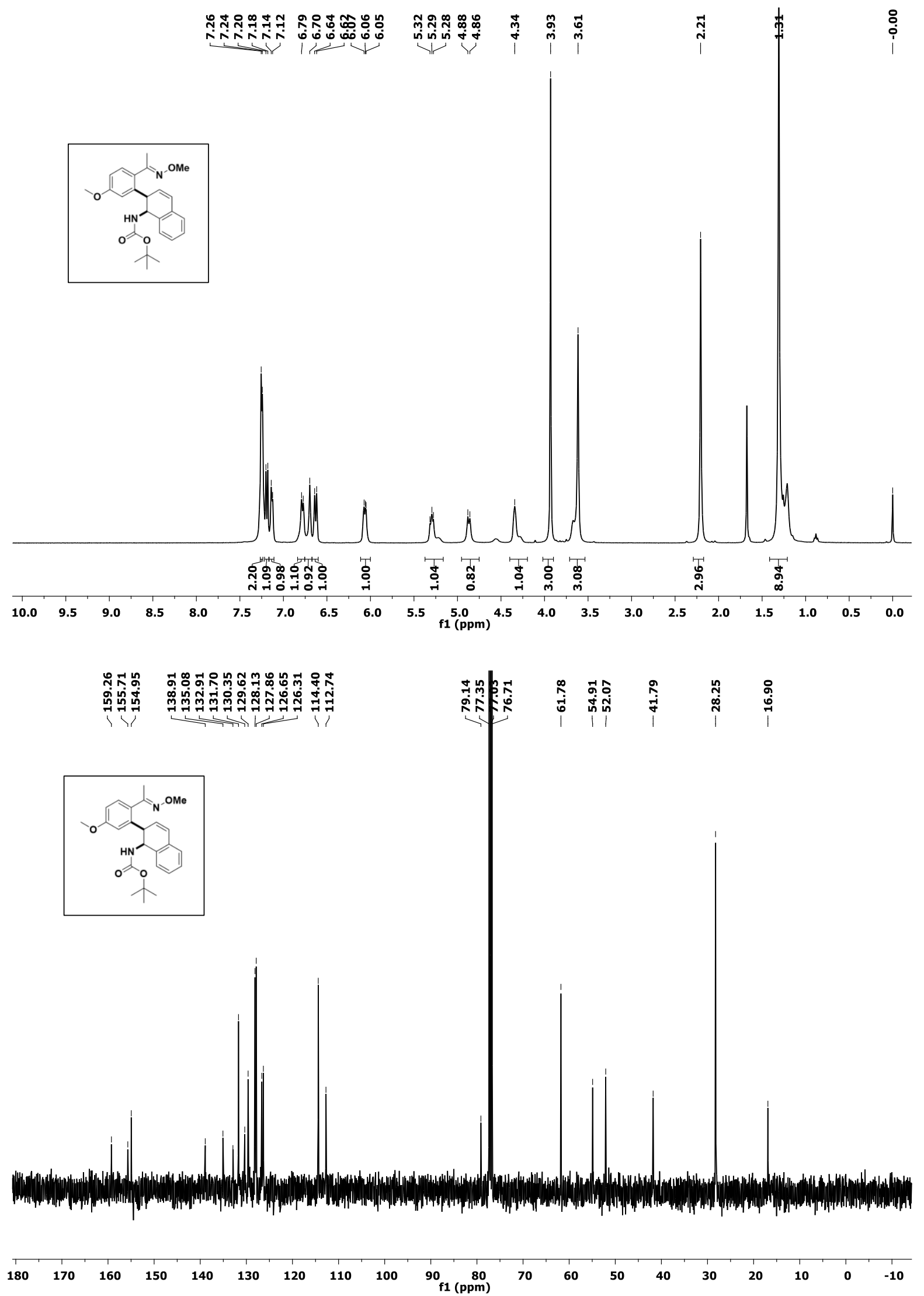
${ }^{1} \mathrm{H}$ and ${ }^{13} \mathrm{C}$ NMR Spectra of Compound 3ca.

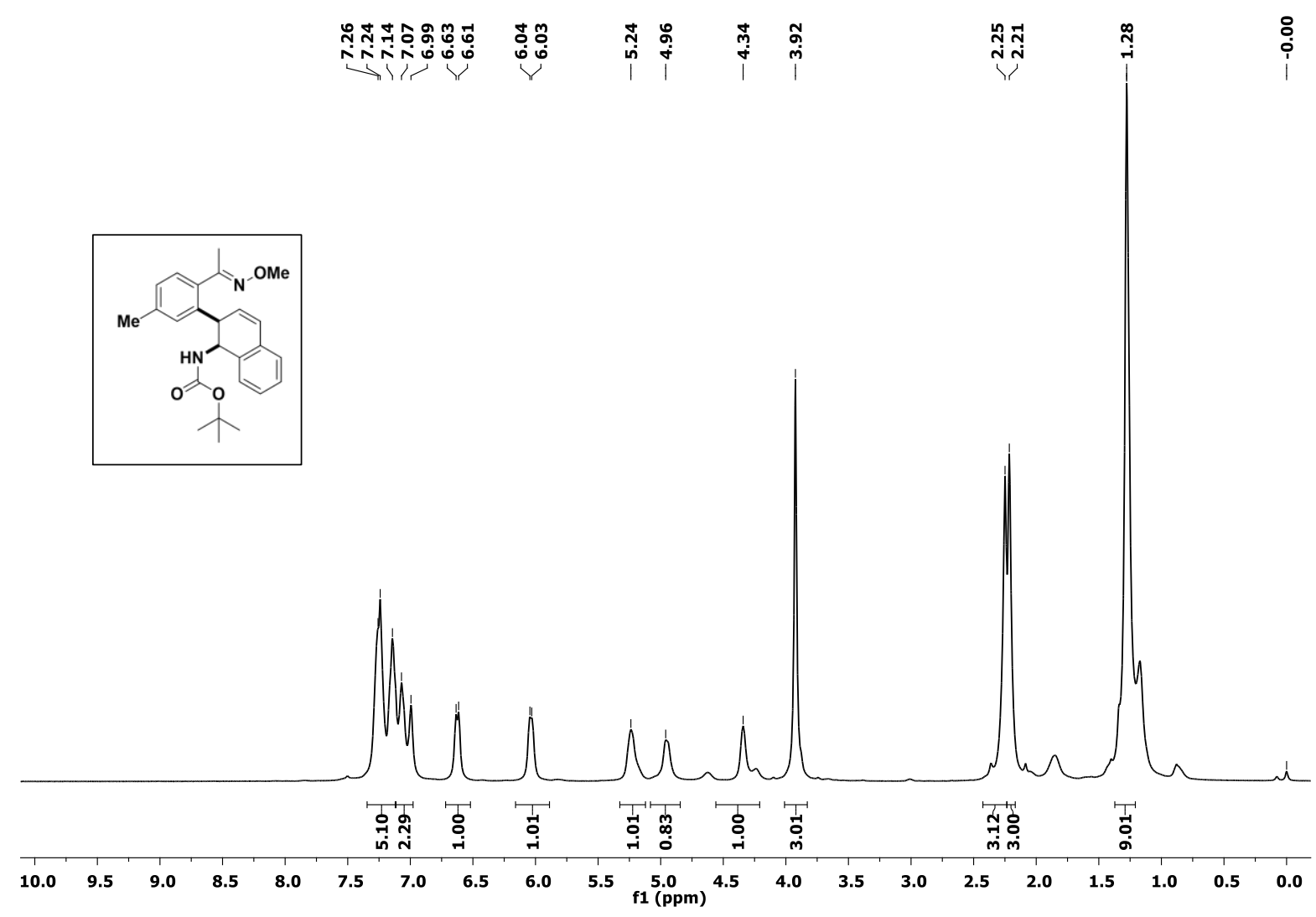

The NMR was taken at $50{ }^{\circ} \mathrm{C}$. The bumps range $4.6 \mathrm{ppm}$ and $4.2 \mathrm{ppm}$ were disappeared

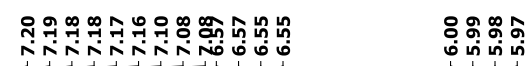

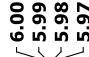

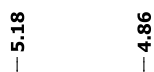

$\stackrel{\text { ริ }}{1}$
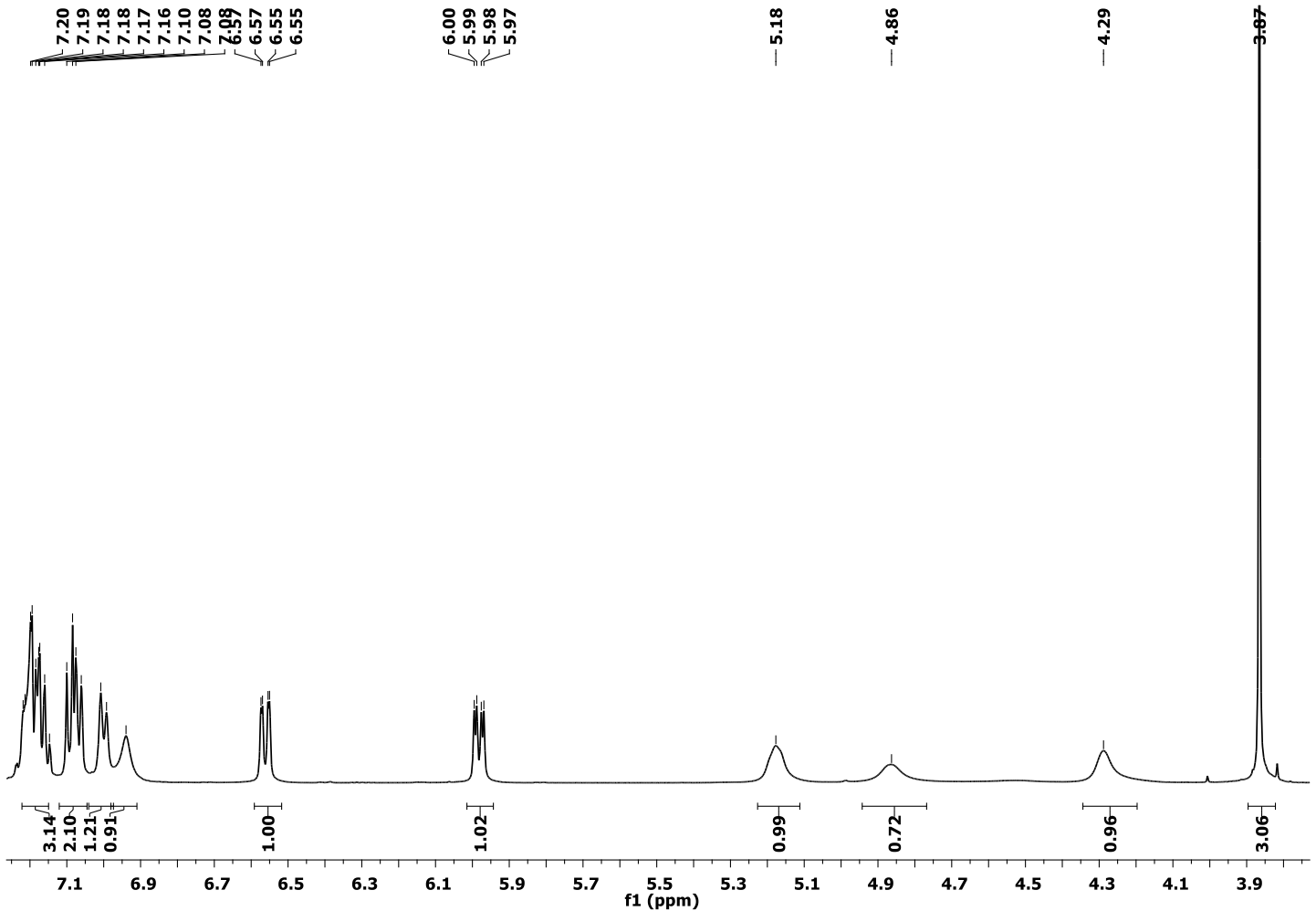


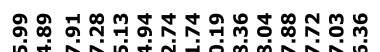

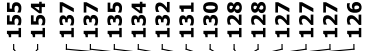

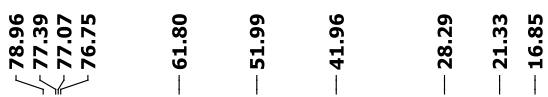

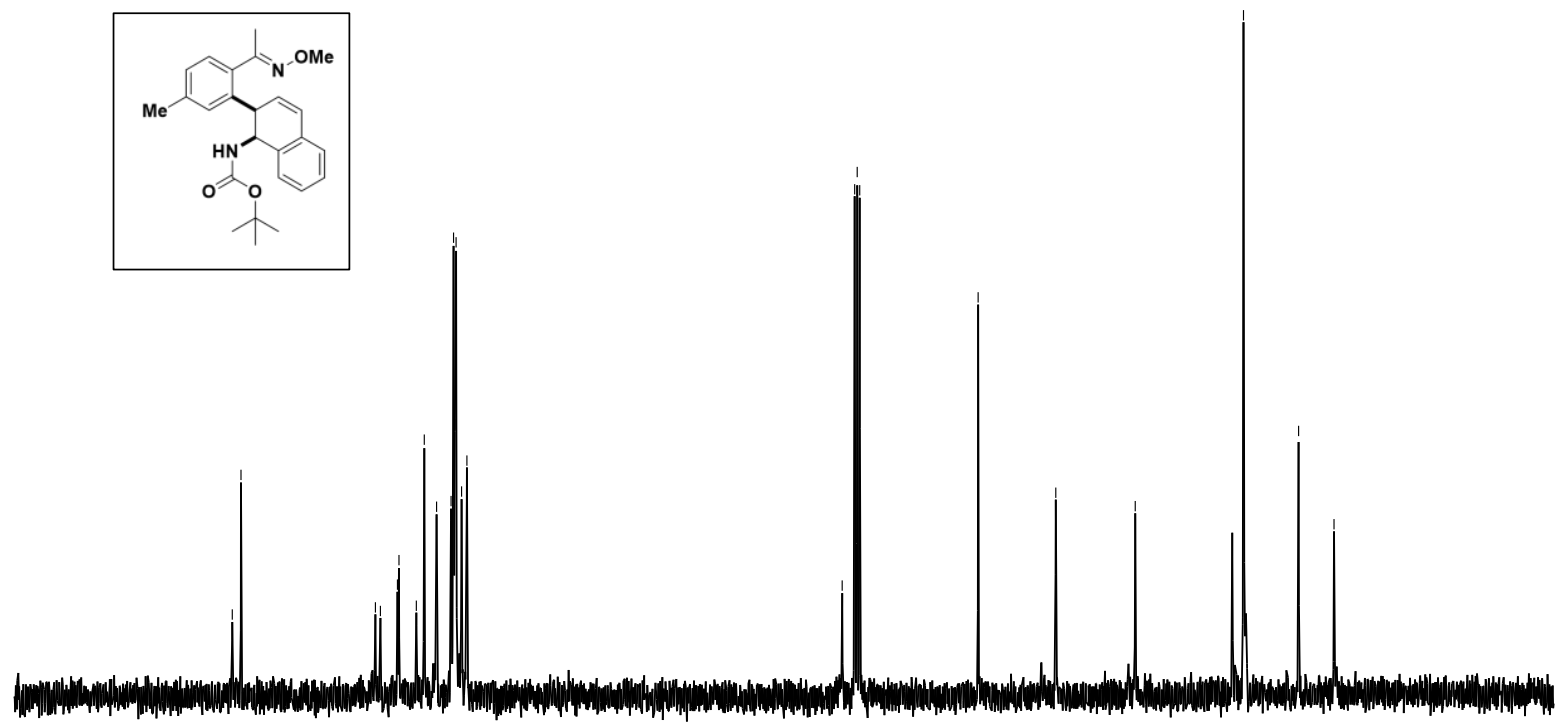

$\begin{array}{llllllllllllllllllll}180 & 170 & 160 & 150 & 140 & 130 & 120 & 110 & 100 & \underset{f 1}{90}(\mathrm{ppm}) & 80 & 70 & 60 & 50 & 40 & 30 & 20 & 10 & 0 & -10\end{array}$ 
${ }^{1} \mathrm{H}$ and ${ }^{13} \mathrm{C}$ NMR Spectra of Compound 3da.

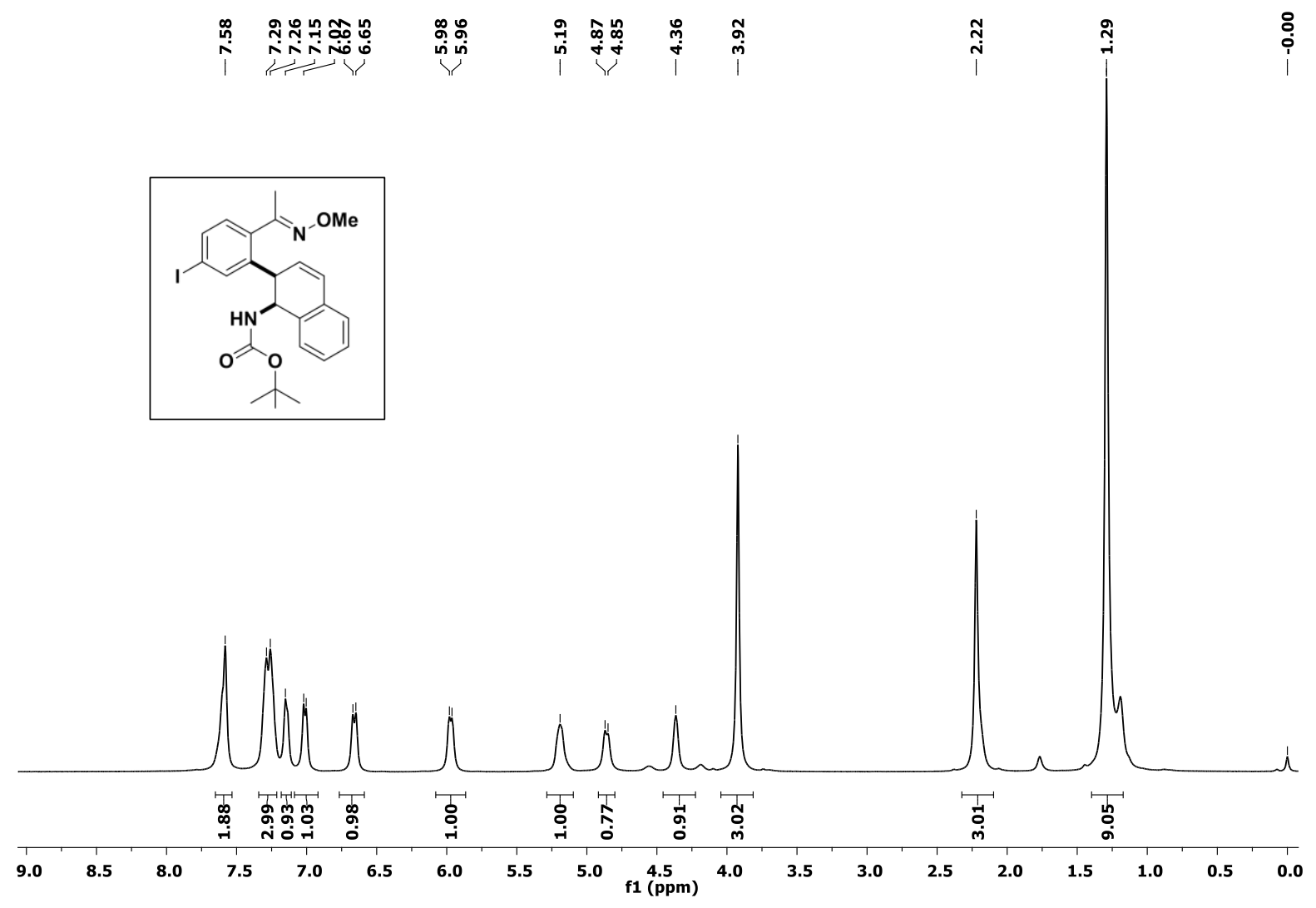

Fine shimming NMR: expended Chemical shift $7.60 \mathrm{ppm}$ to $3.8 \mathrm{ppm}$ for better splitting 我象品

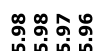

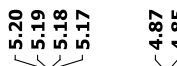

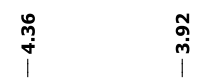

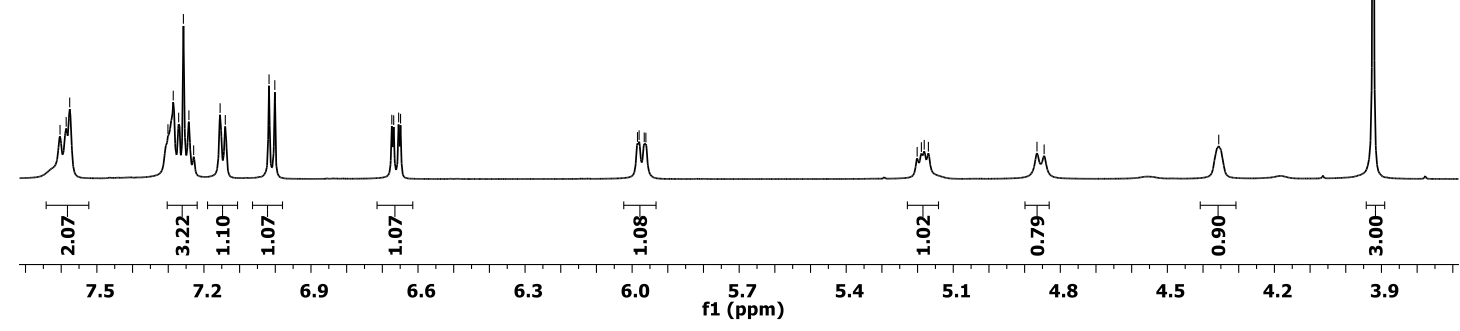



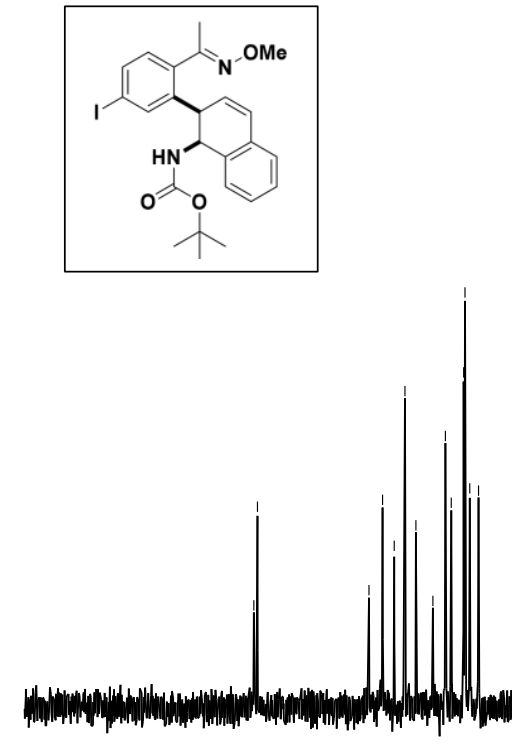

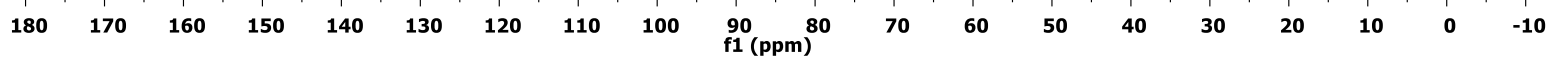


${ }^{1} \mathrm{H}$ and ${ }^{13} \mathrm{C}$ NMR Spectra of Compound 3ea.
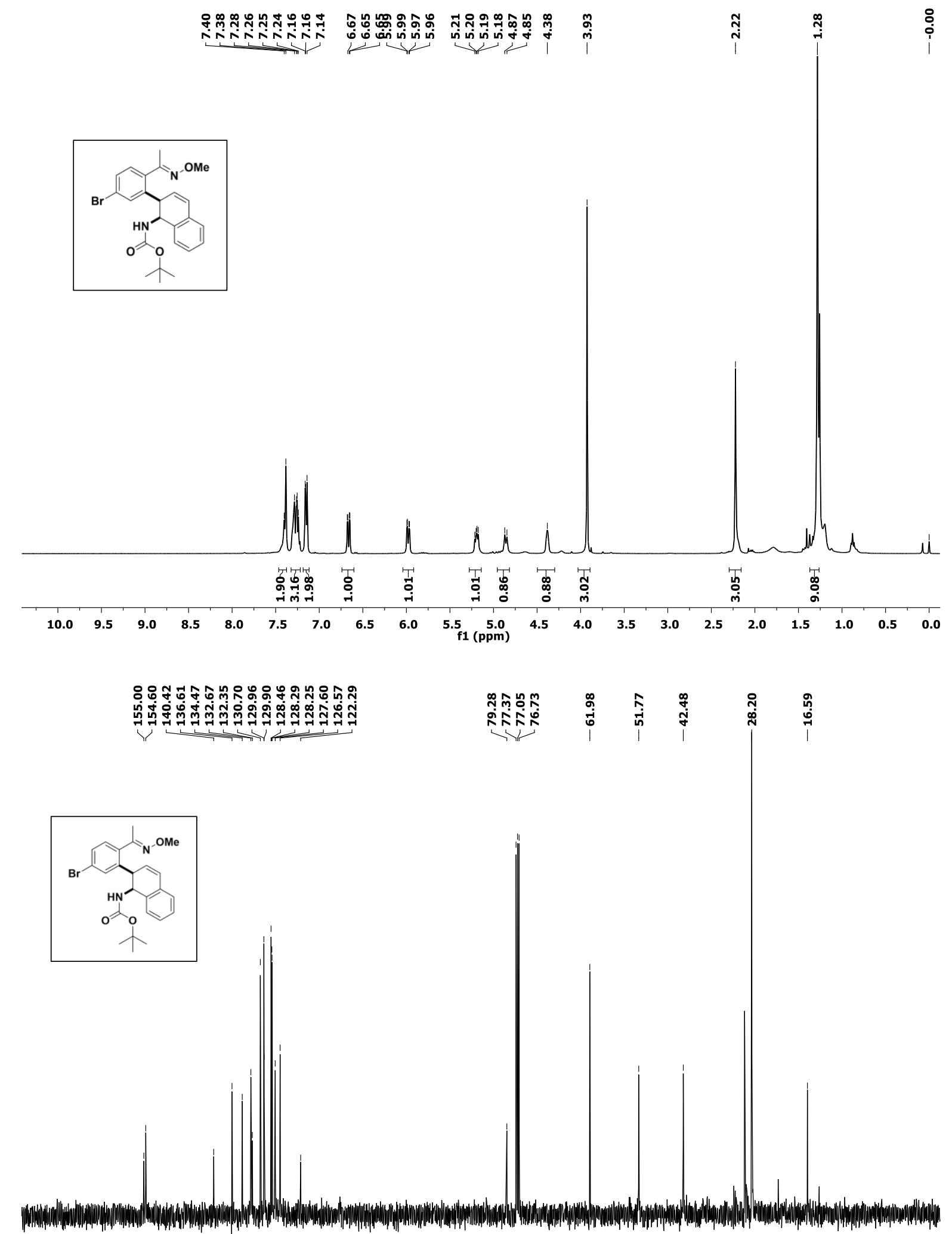

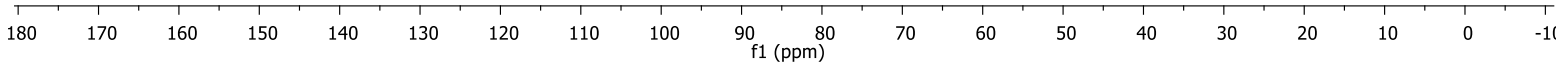


${ }^{1} \mathrm{H}$ and ${ }^{13} \mathrm{C}$ NMR Spectra of Compound $3 \mathbf{f a}$.
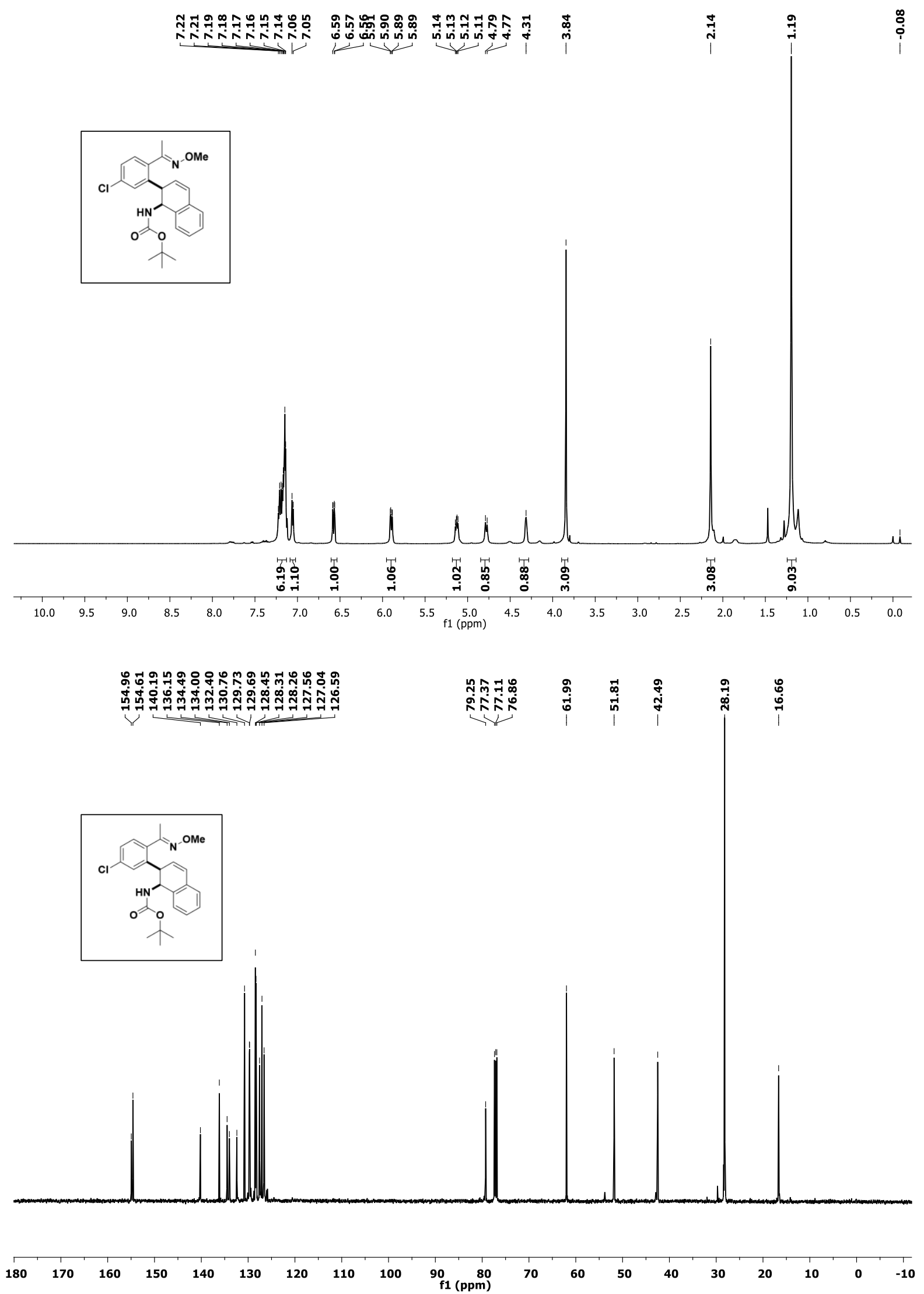
${ }^{1} \mathrm{H}$ and ${ }^{13} \mathrm{C}$ NMR Spectra of Compound 3ga.
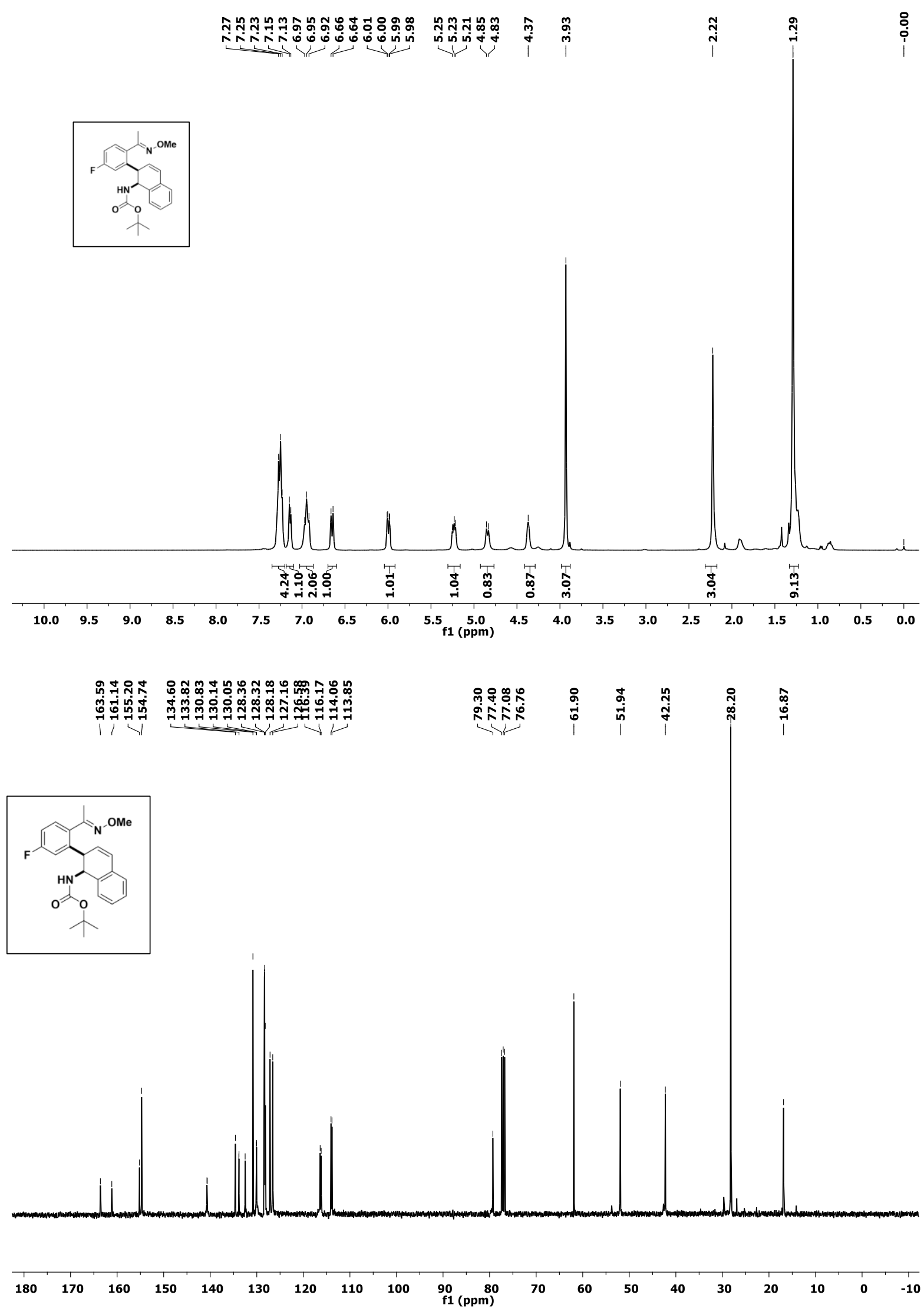
${ }^{1} \mathrm{H}$ and ${ }^{13} \mathrm{C}$ NMR Spectra of Compound 3ha.

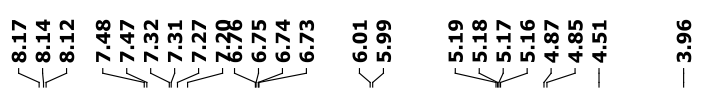

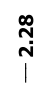
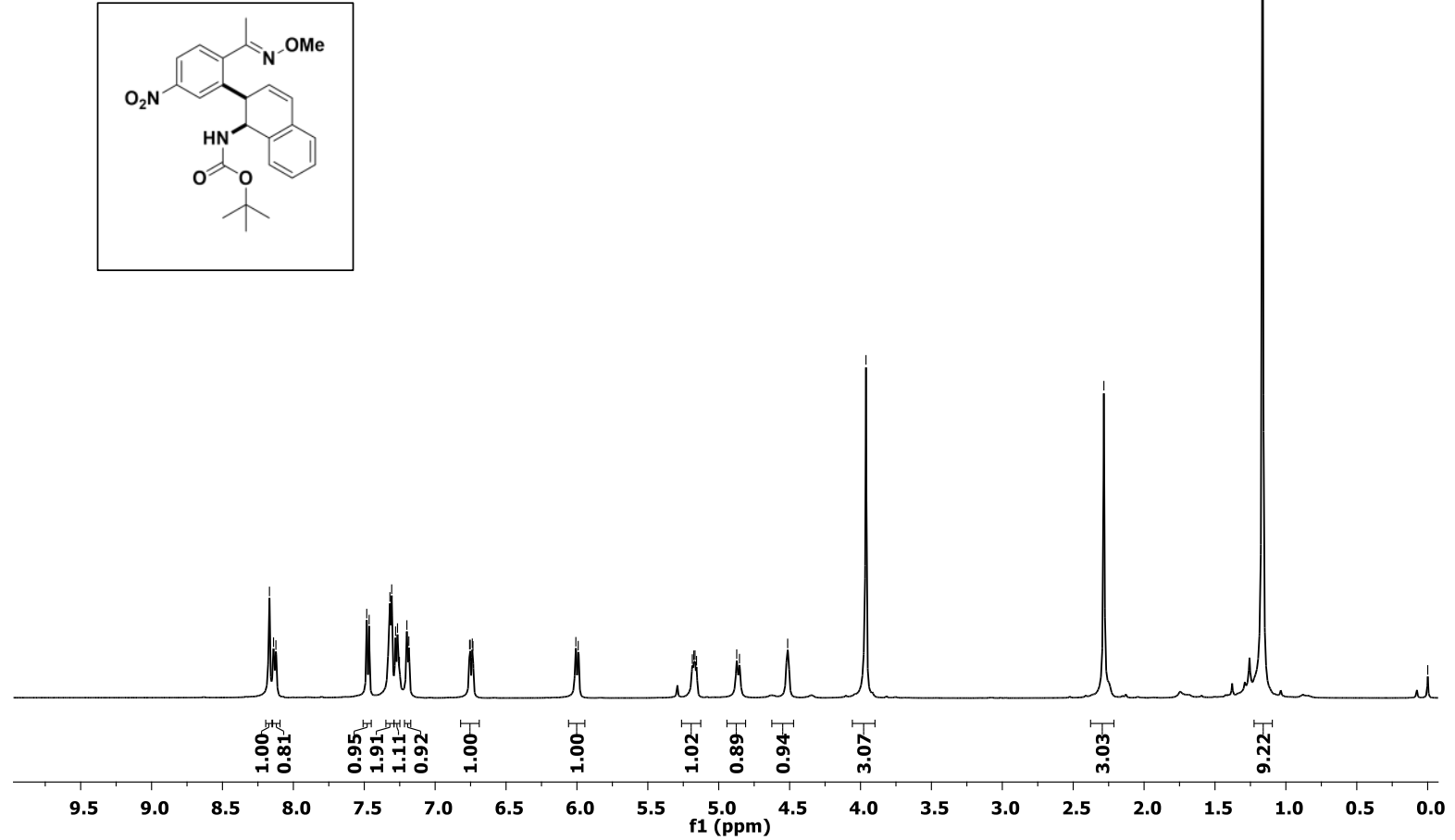

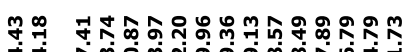

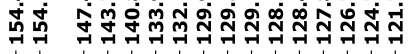

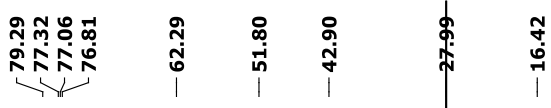
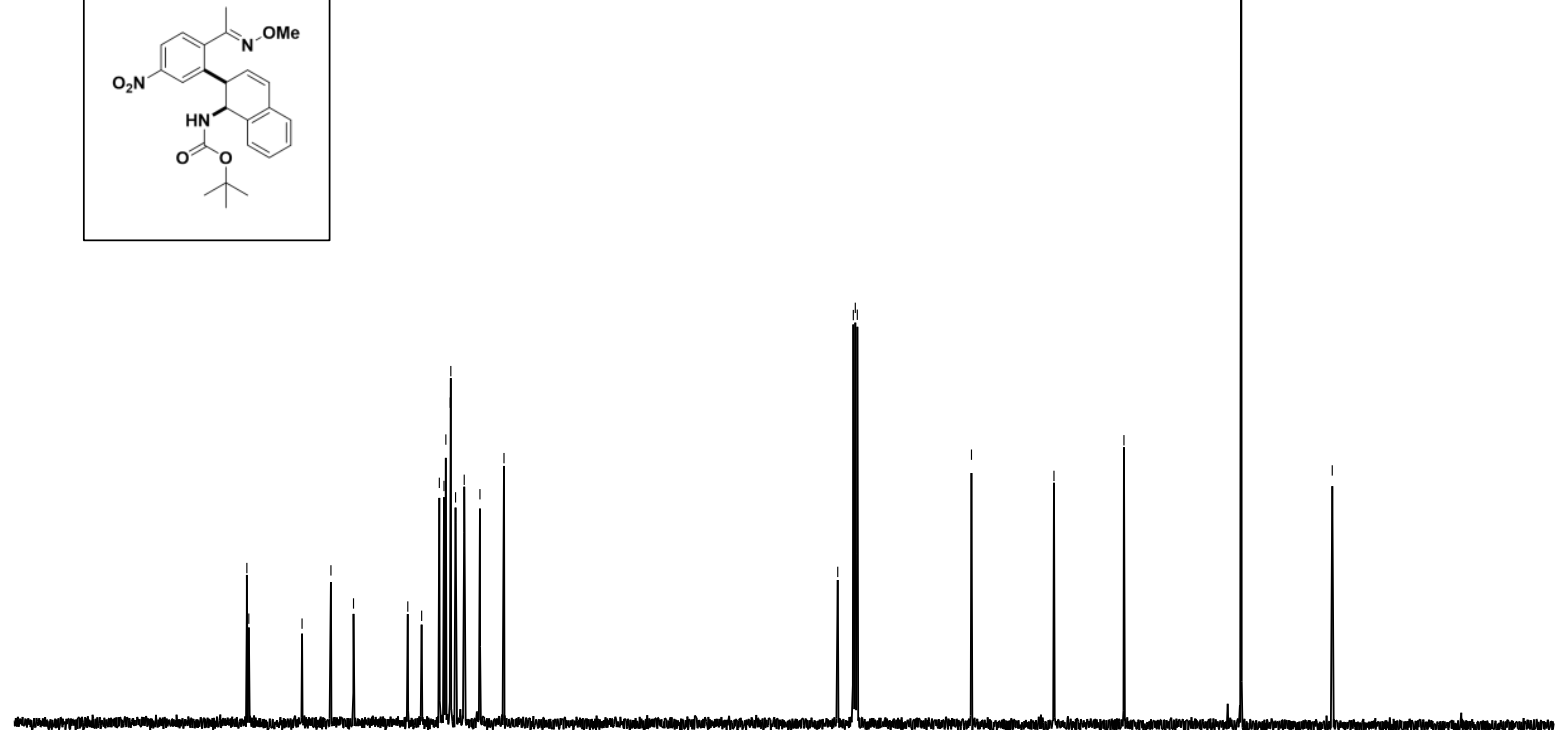
${ }^{1} \mathrm{H}$ and ${ }^{13} \mathrm{C}$ NMR Spectra of Compound 3ia.

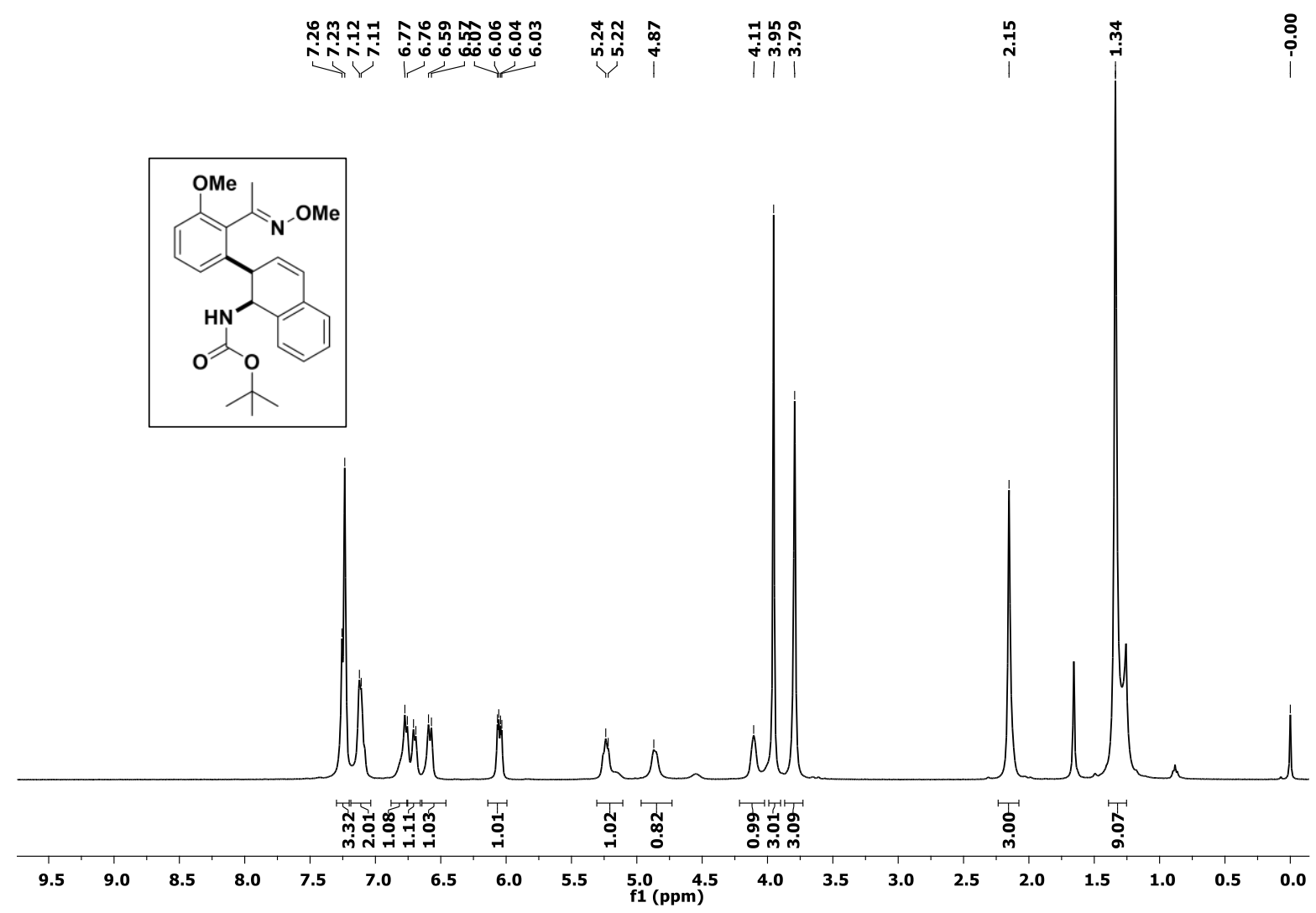

The NMR was taken at $50{ }^{\circ} \mathrm{C}$. The bumps range $5.2 \mathrm{ppm}$ and $4.5 \mathrm{ppm}$ were disappeared

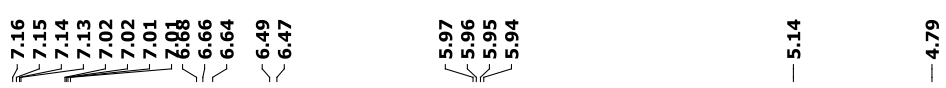

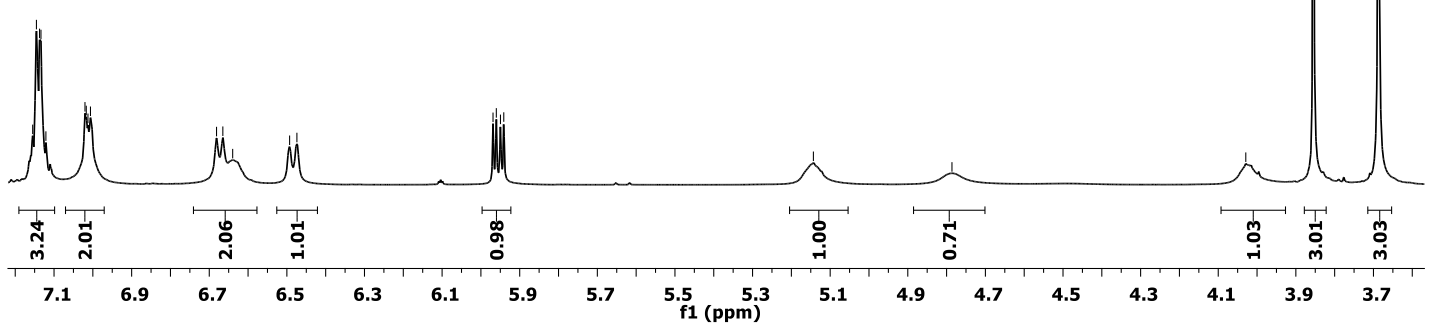




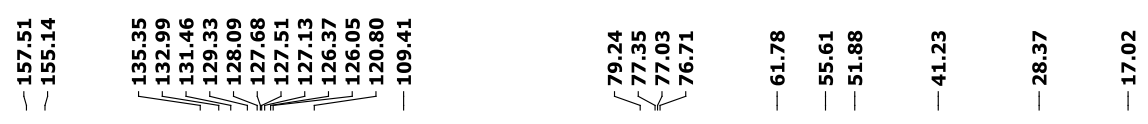

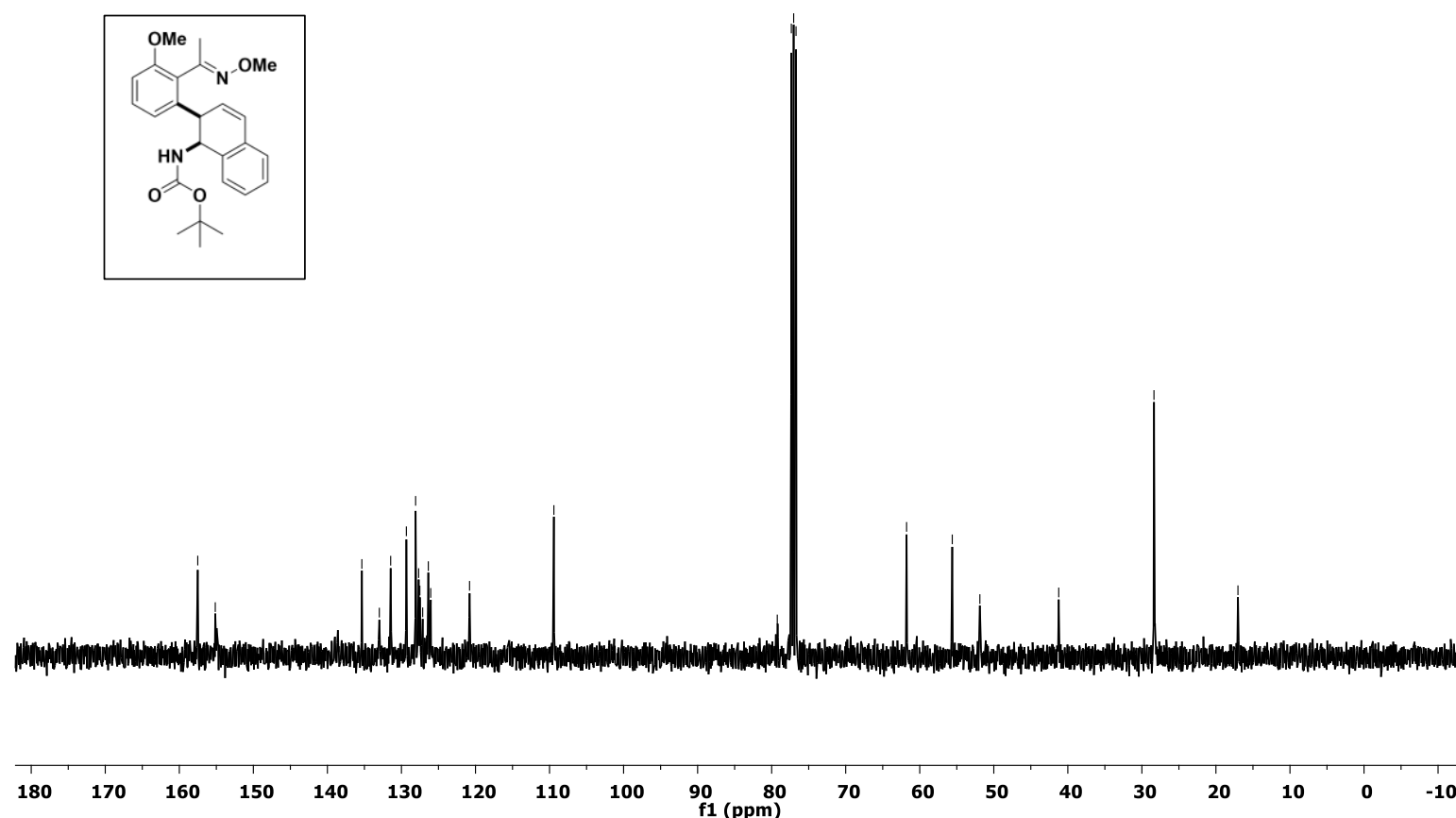


${ }^{1} \mathrm{H}$ and ${ }^{13} \mathrm{C}$ NMR Spectra of Compound 3ja.

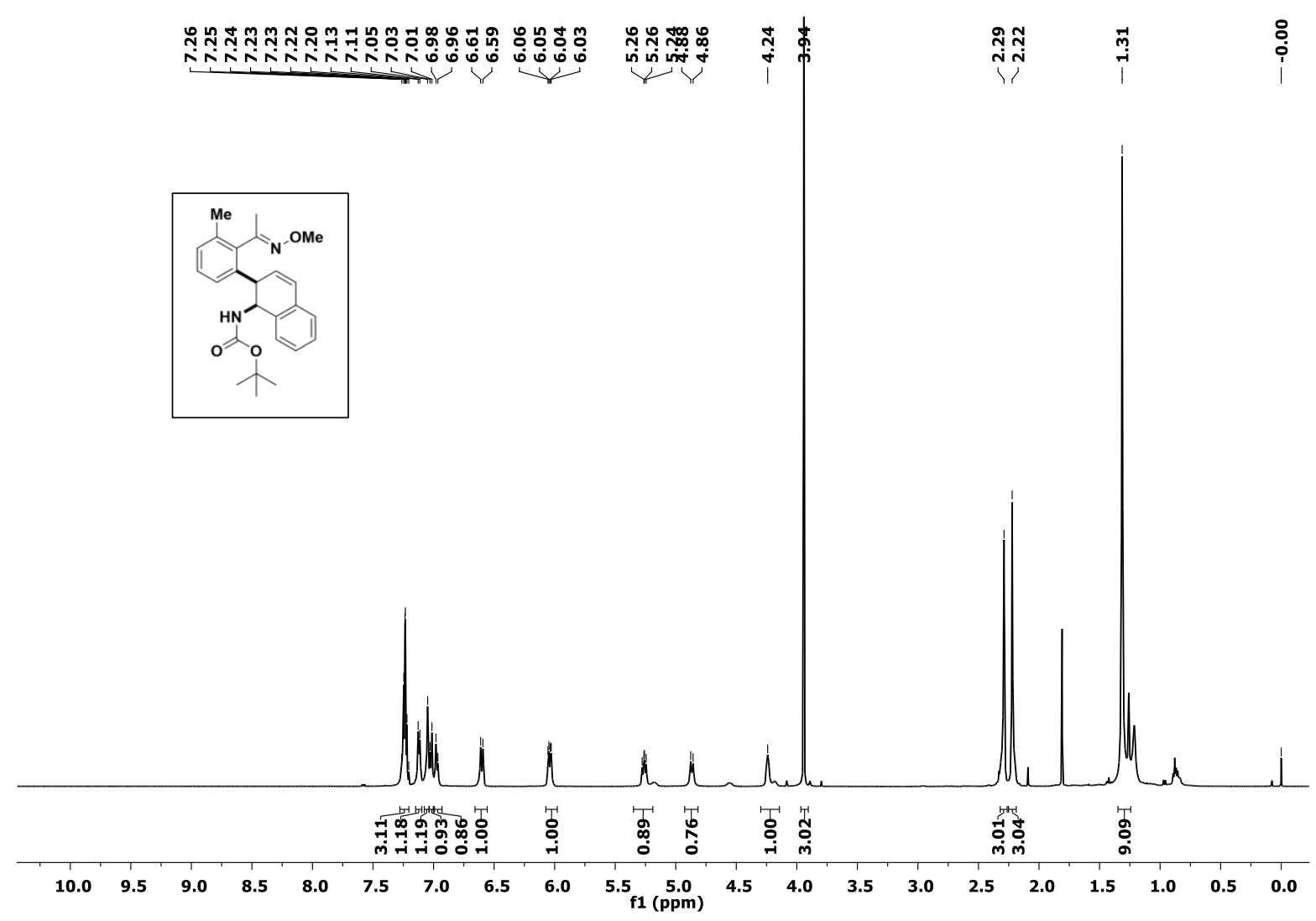

Fine shimming NMR: expended Chemical shift $7.30 \mathrm{ppm}$ to $3.9 \mathrm{ppm}$ for better splitting

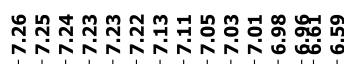

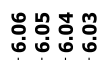

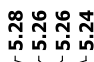
ฌั:

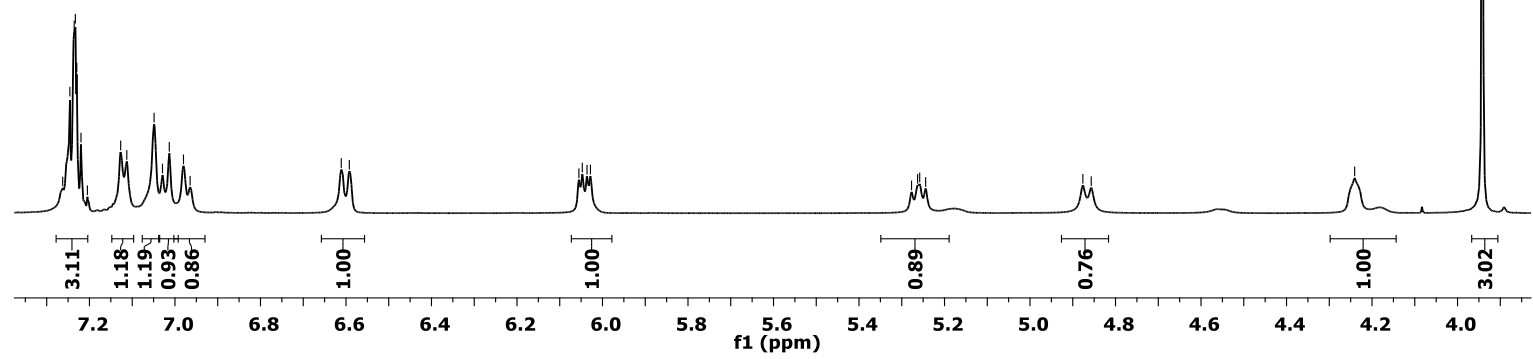



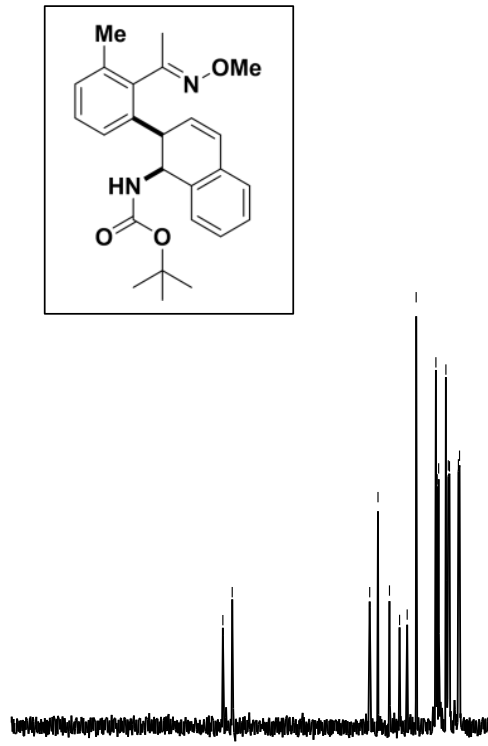

$180 \quad 170$

$\begin{array}{lll}150 & 140 \quad 130\end{array}$

f1 (ppm) 
${ }^{1} \mathrm{H}$ and ${ }^{13} \mathrm{C}$ NMR Spectra of Compound 3ka.

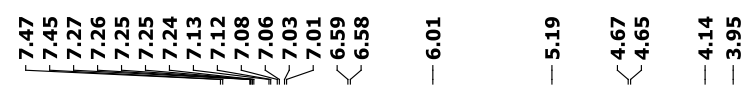

$\stackrel{\text { i }}{\text { i }}$

$\stackrel{8}{i}$
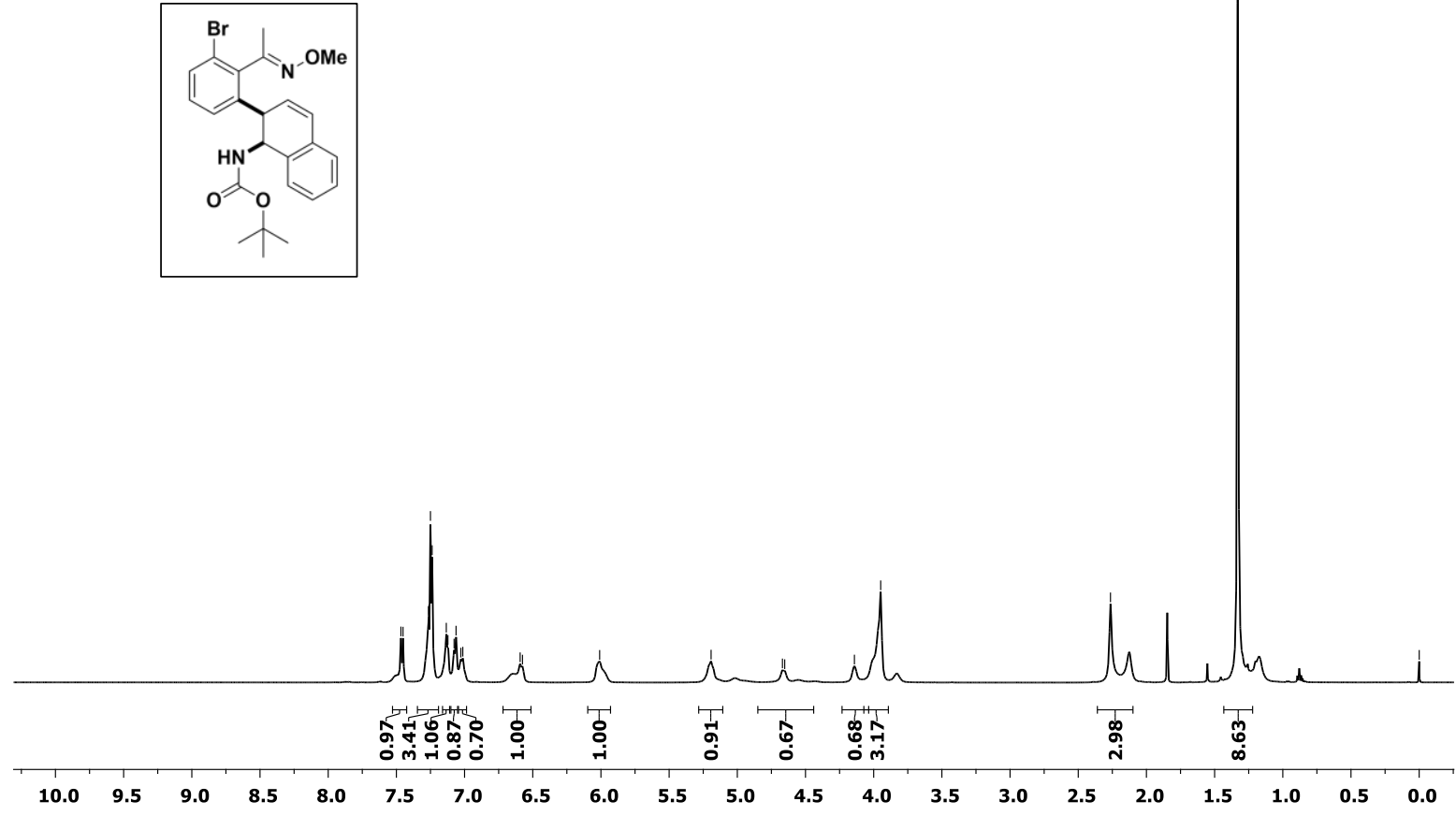

Fine shimming NMR expended: Chemical shift 7.60 ppm to $3.8 \mathrm{ppm}$ for better splitting

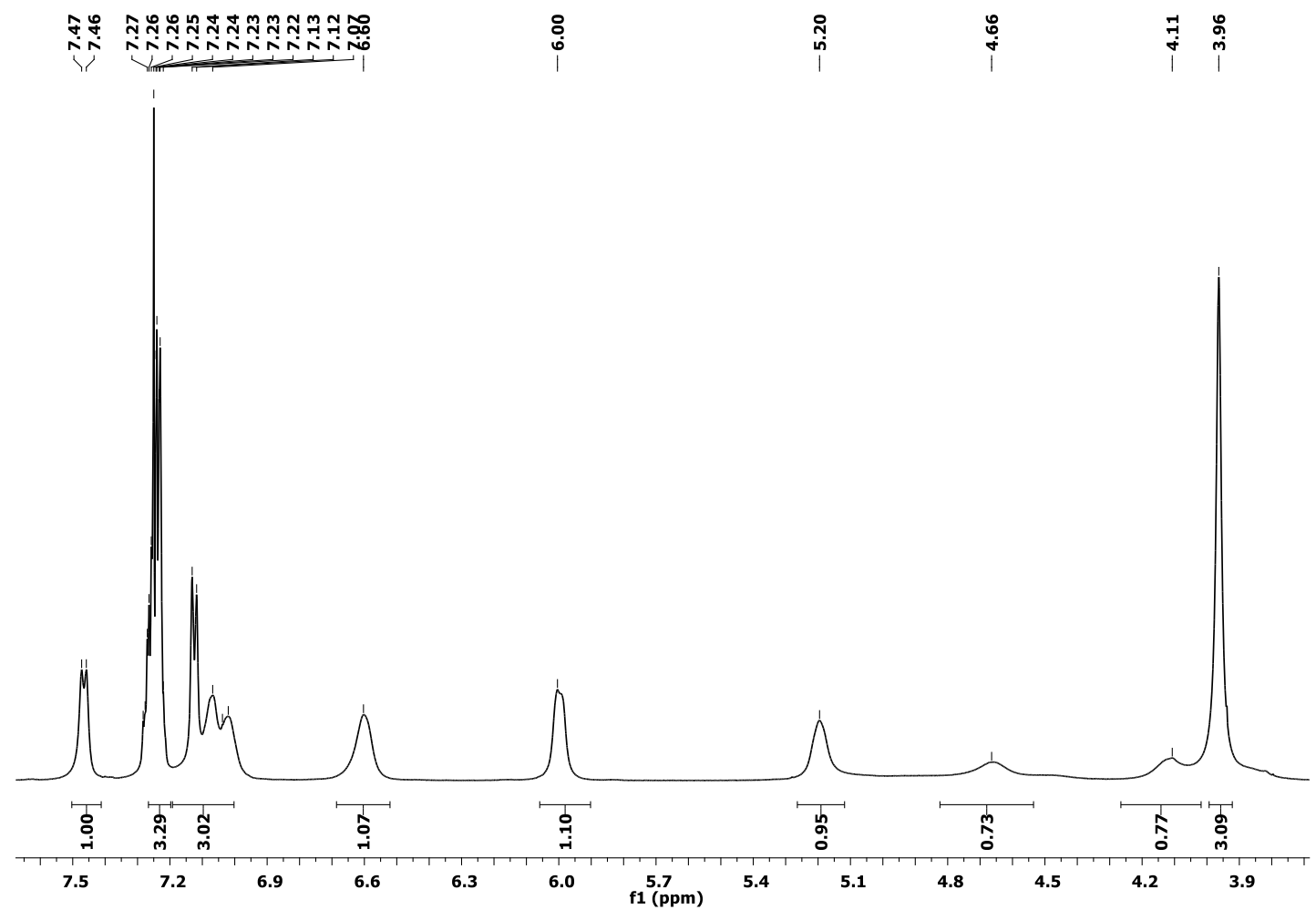




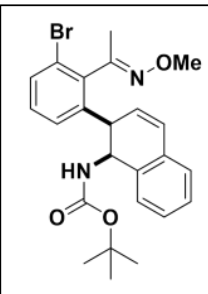


${ }^{1} \mathrm{H}$ and ${ }^{13} \mathrm{C}$ NMR Spectra of Compound 3la.

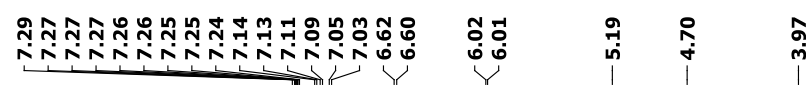

$\stackrel{\sim}{i}$

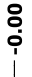
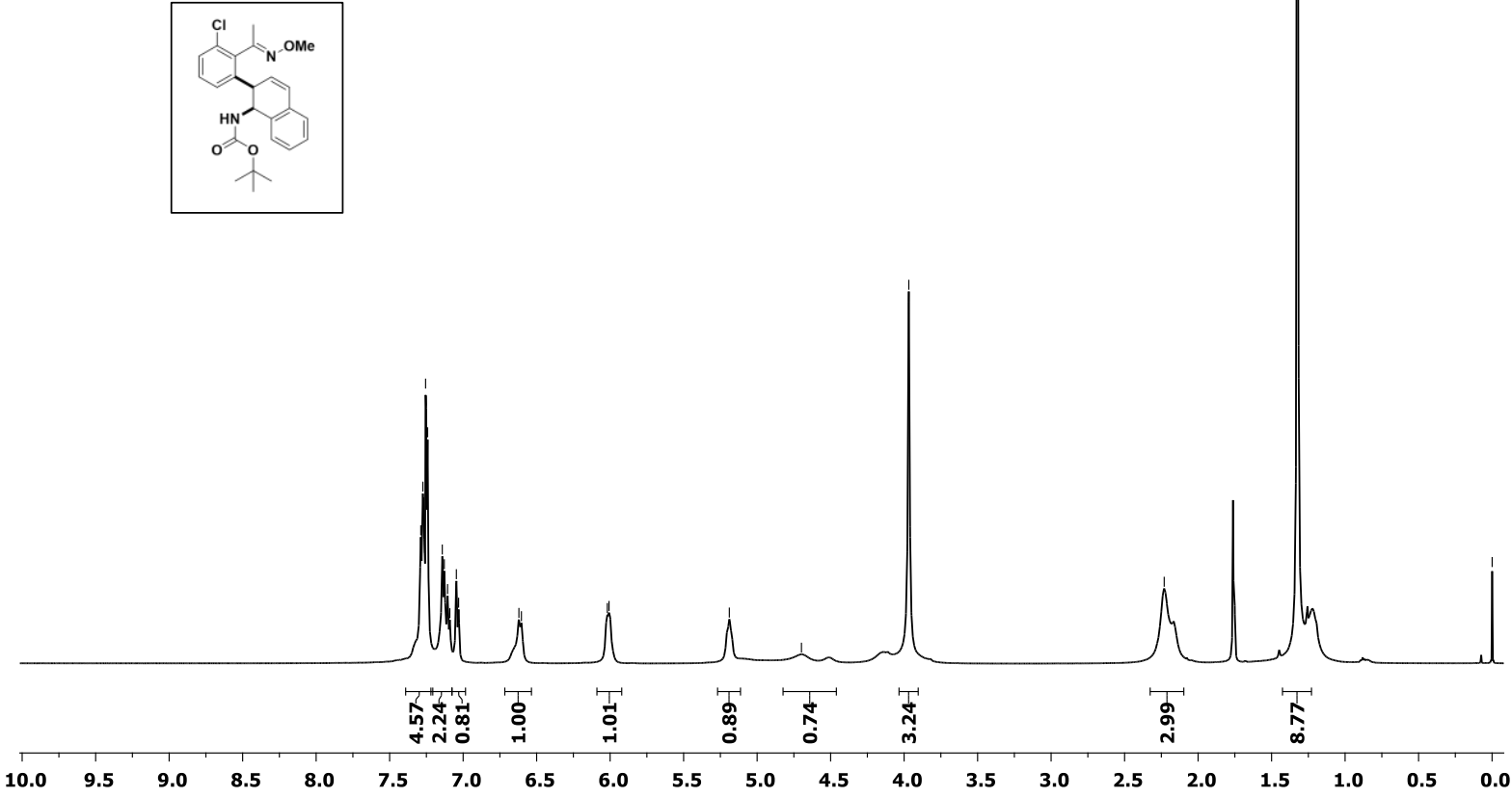

Fine shimming NMR expended: Chemical shift $7.30 \mathrm{ppm}$ to $3.9 \mathrm{ppm}$ for better splitting

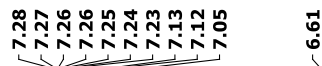

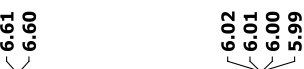

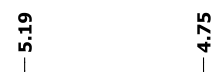

ò

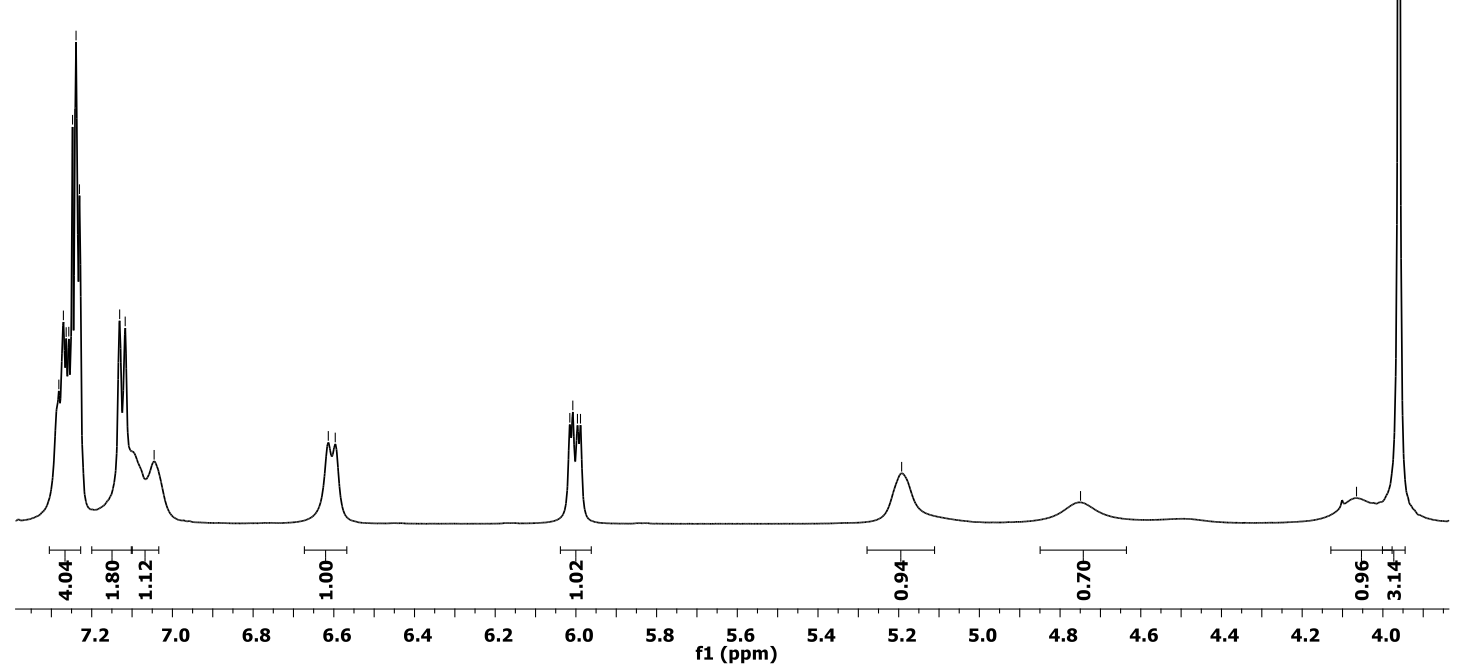




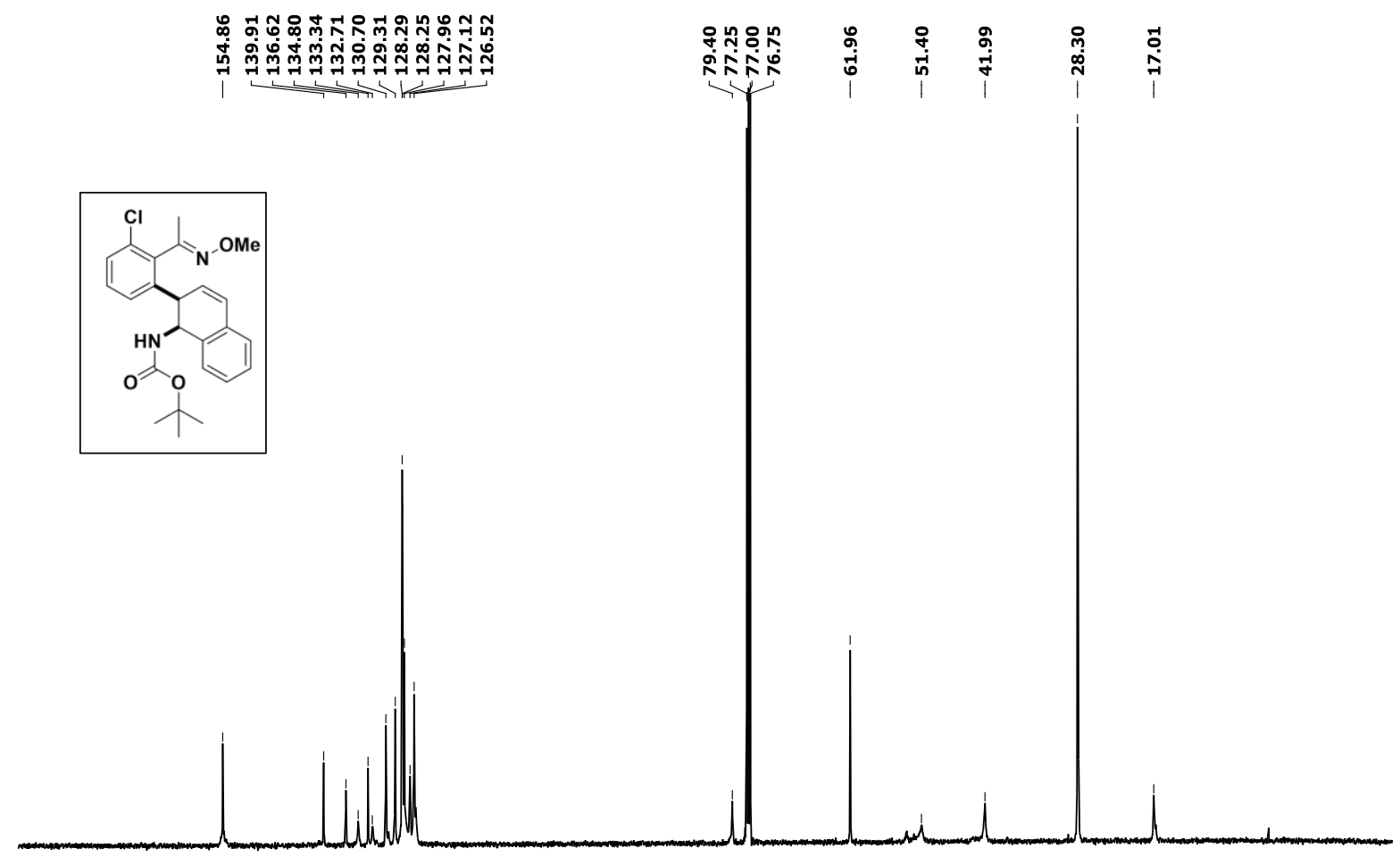

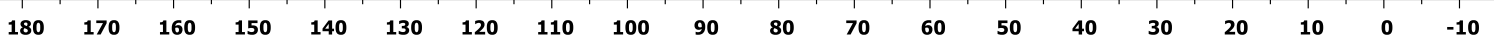


${ }^{1} \mathrm{H}$ and ${ }^{13} \mathrm{C}$ NMR Spectra of Compound 3ma.

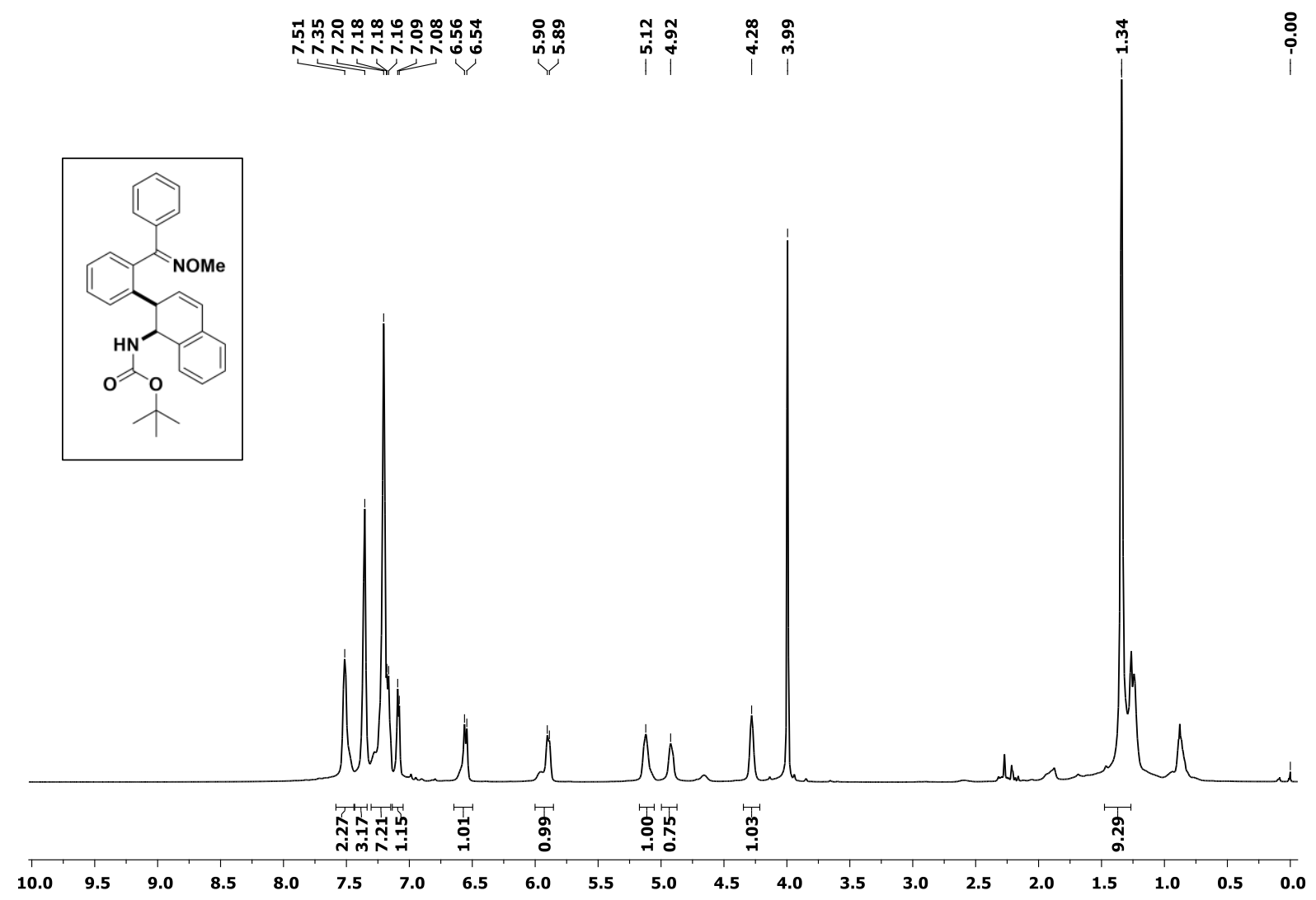

The NMR was taken at $50{ }^{\circ} \mathrm{C}$. The bumps range $5.9 \mathrm{ppm}$ and $4.6 \mathrm{ppm}$ were disappeared

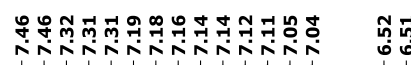

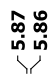

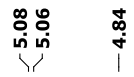

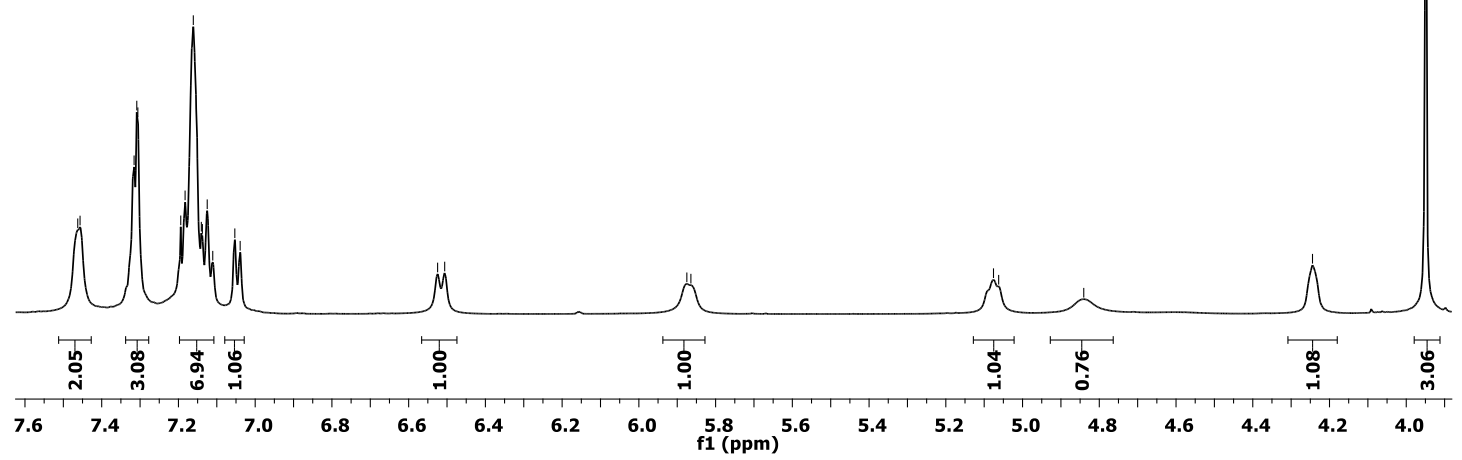




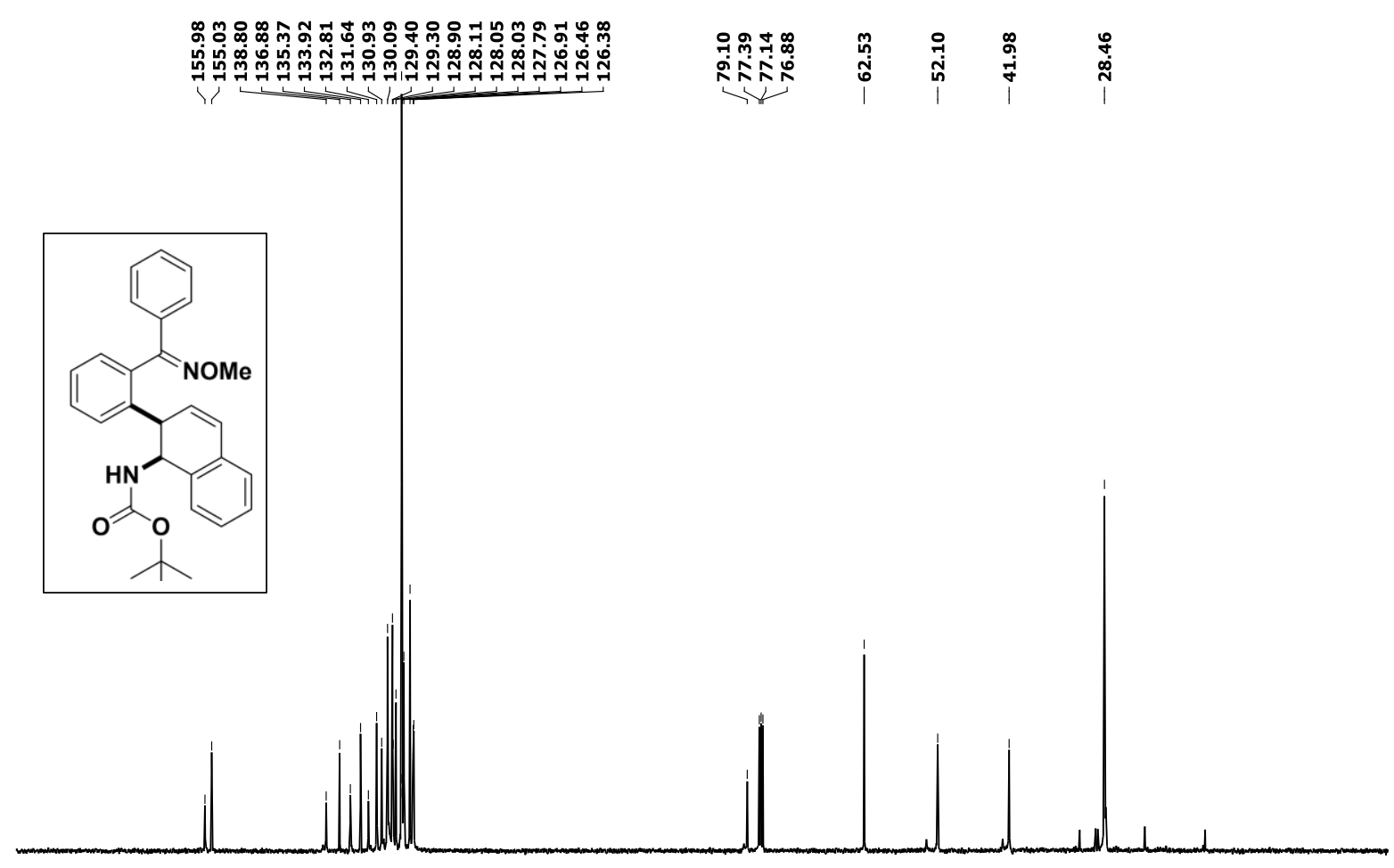

$\begin{array}{lllllllllllllllllllll}180 & 170 & 160 & 150 & 140 & 130 & 120 & 110 & 100 & 90 & 80 & 70 & 60 & 50 & 40 & 30 & 20 & 10 & 0 & -10\end{array}$ 
${ }^{1} \mathrm{H}$ and ${ }^{13} \mathrm{C}$ NMR Spectra of Compound 3na.
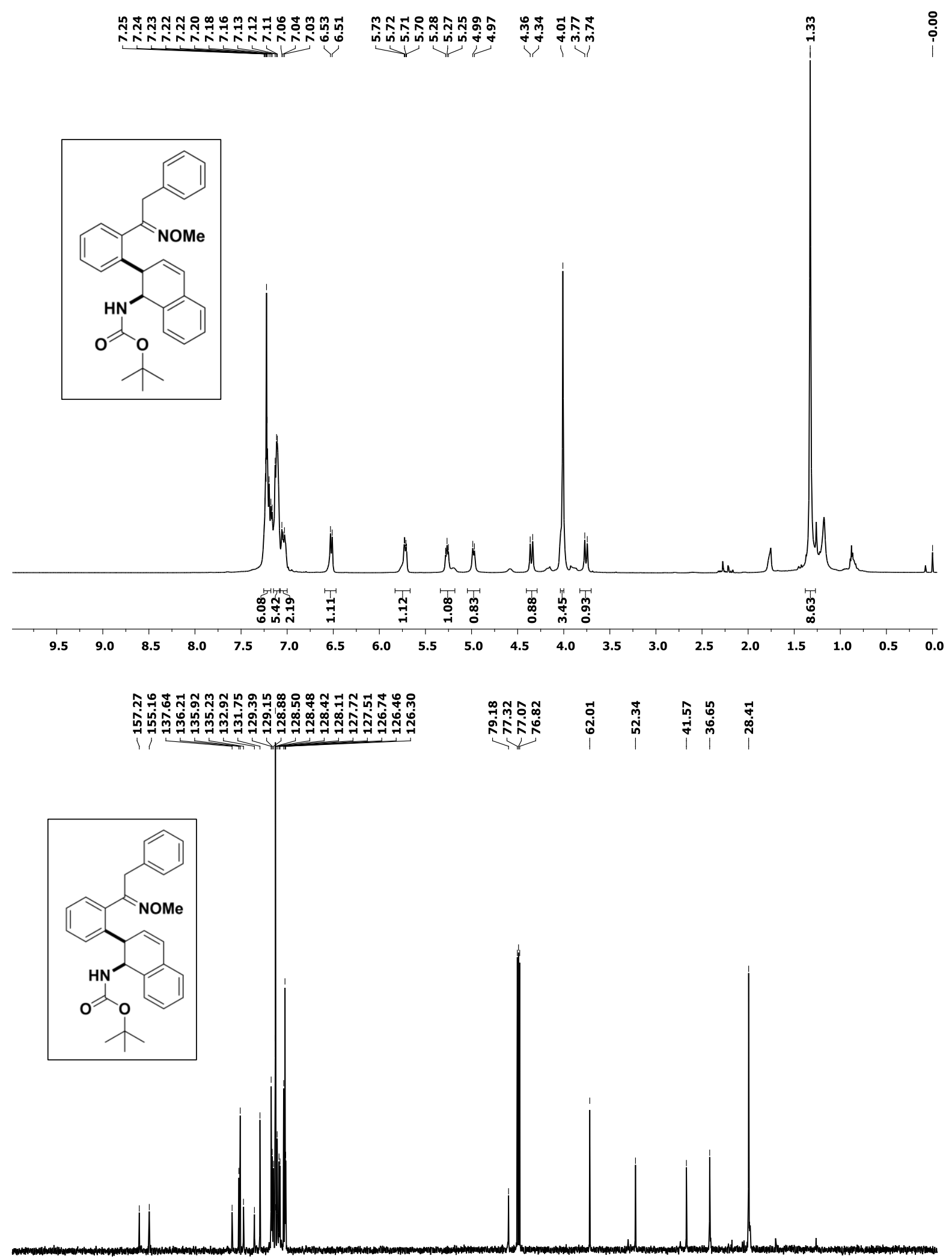

$\begin{array}{llllllllllllllllllllll}180 & 170 & 160 & 150 & 140 & 130 & 120 & 110 & 100 & 90 & 80 & 70 & 60 & 50 & 40 & 30 & 20 & 10 & 0 & -10\end{array}$ 
${ }^{1} \mathrm{H}$ and ${ }^{13} \mathrm{C}$ NMR Spectra of Compound 3oa.
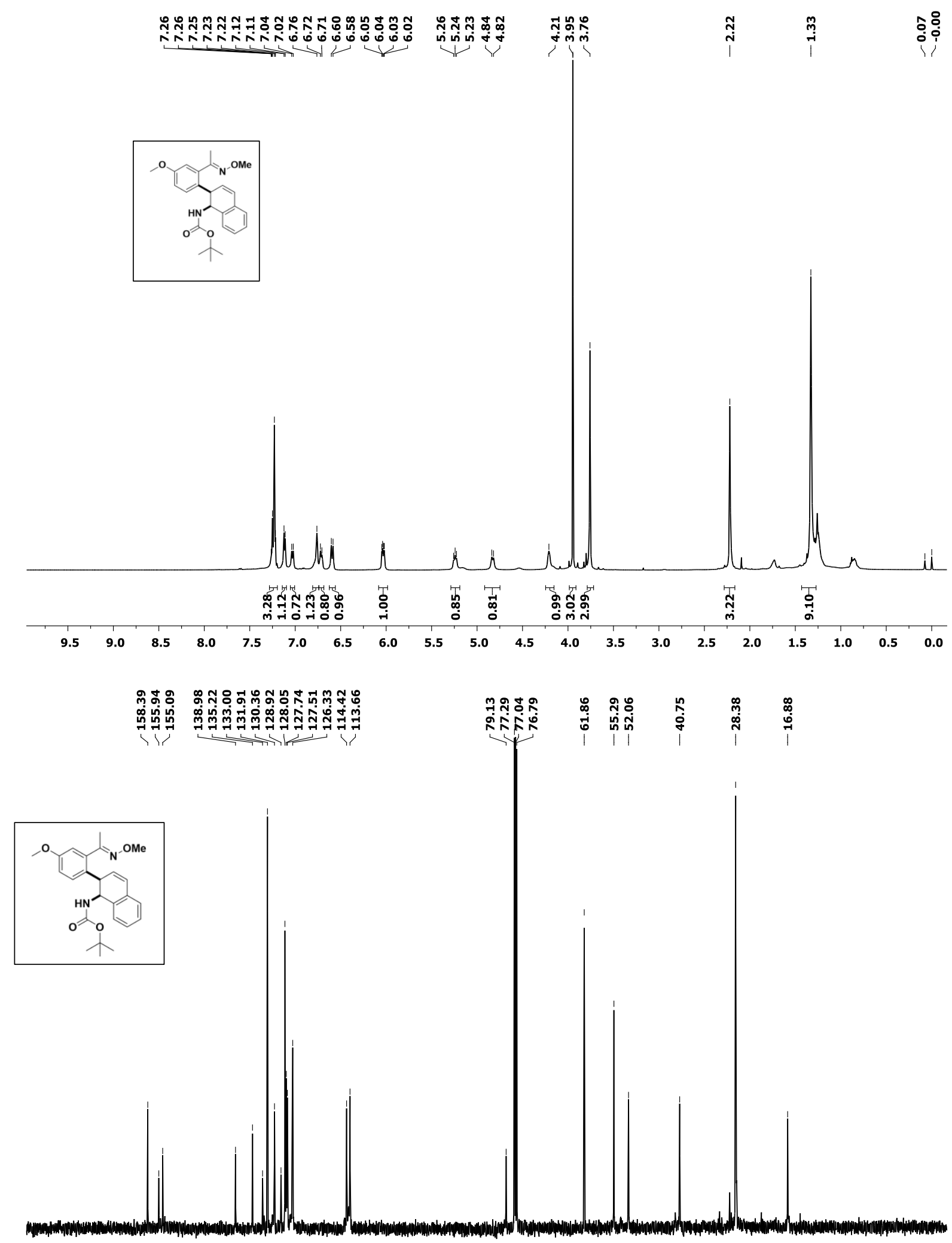

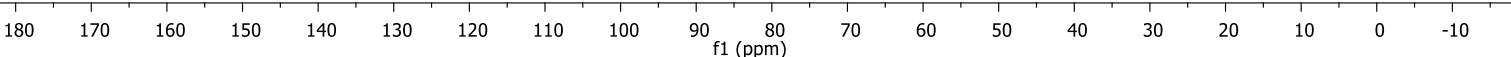


DEPT (135) NMR Spectrum of Compound 3oa.

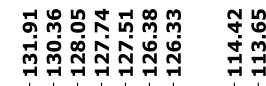

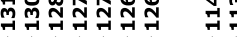

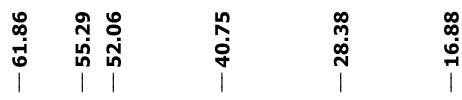
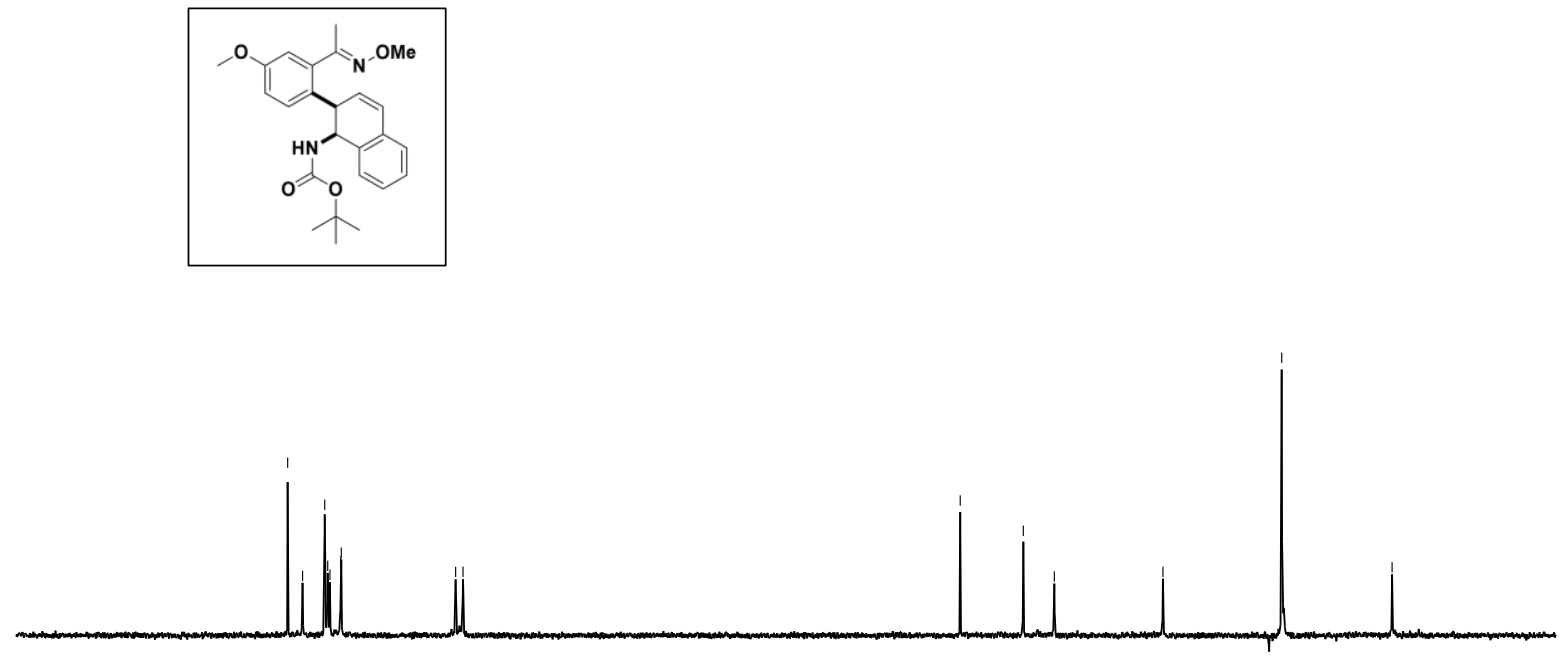

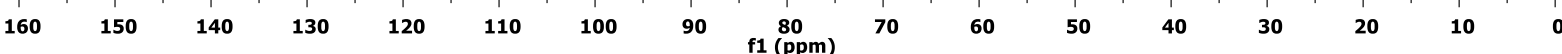


${ }^{1} \mathrm{H}$ and ${ }^{13} \mathrm{C}$ NMR Spectra of Compound 3pa.

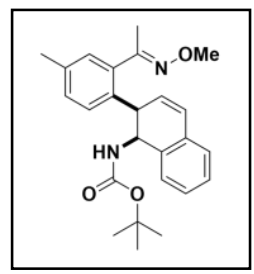

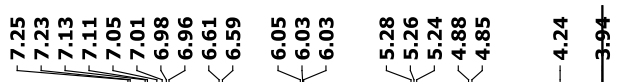

สิกี่

$\stackrel{8}{i}$
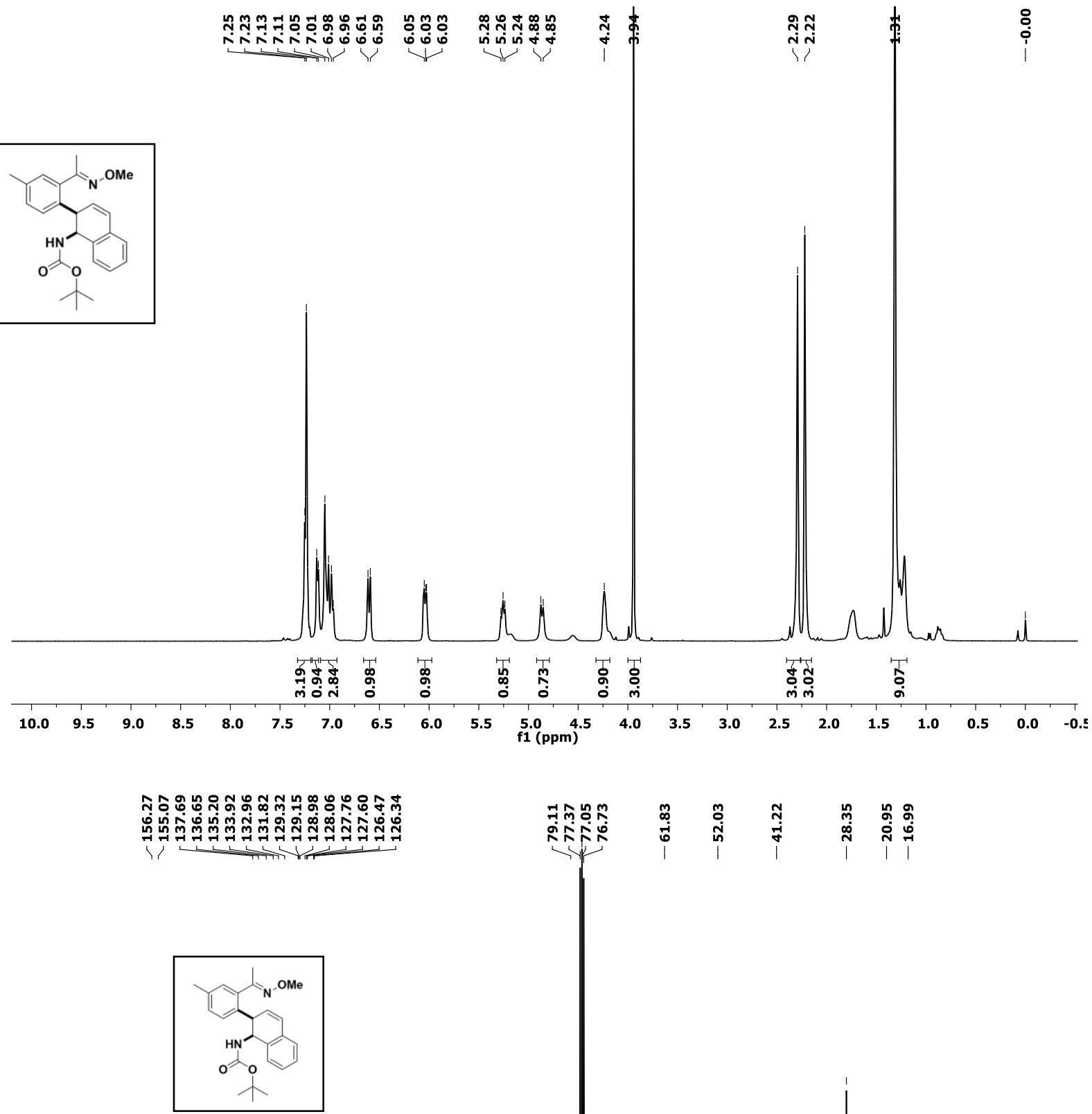

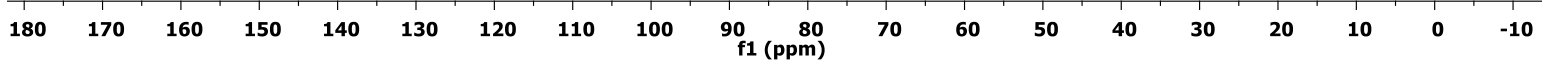

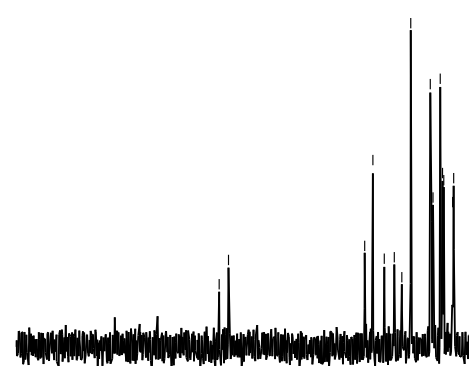


DEPT (135) NMR Spectrum of Compound 3pa.

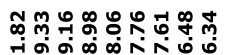

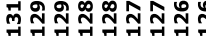

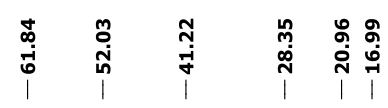
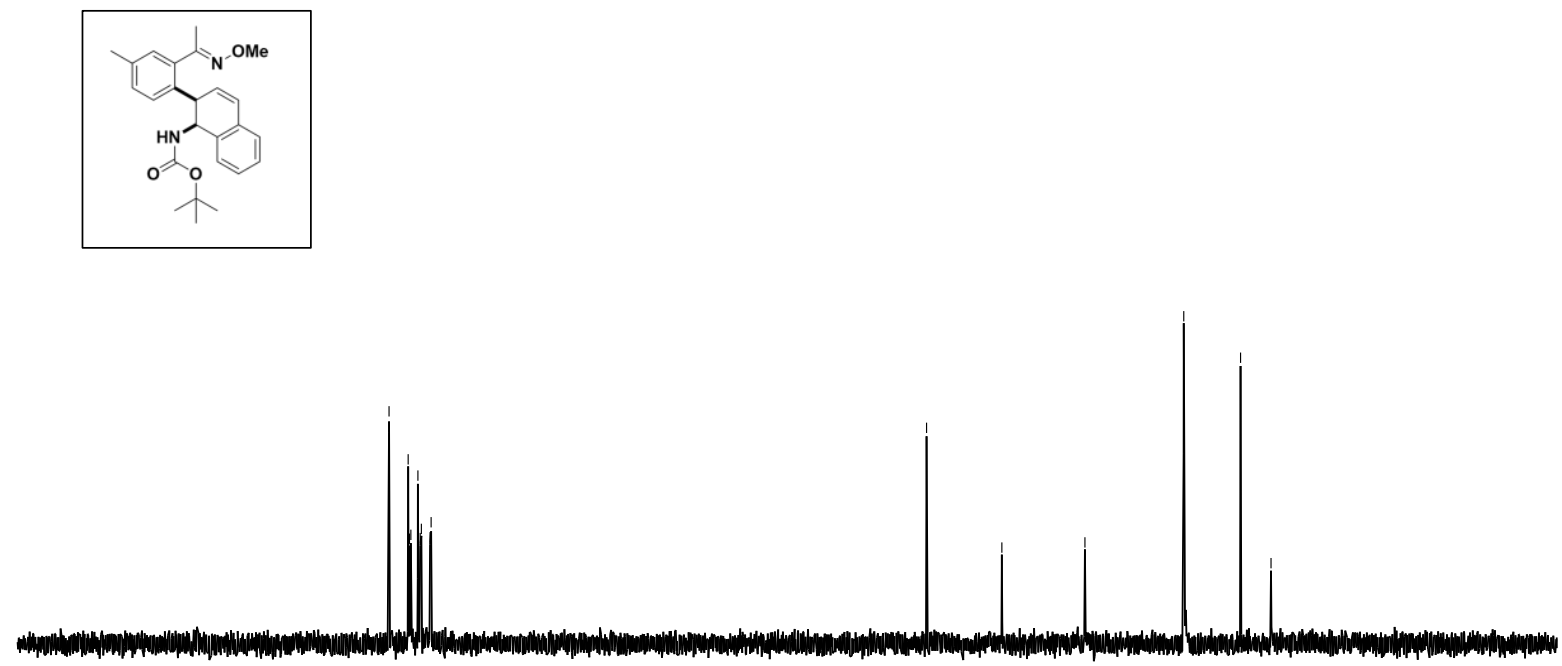

$\begin{array}{lllllllllllllllllllll}180 & 170 & 160 & 150 & 140 & 130 & 120 & 110 & 100 & 90 & \underset{f 1}{80}(\mathrm{ppm}) & 70 & 60 & 50 & 40 & 30 & 20 & 10 & 0 & -10 & -2 C\end{array}$ 
${ }^{1} \mathrm{H}$ and ${ }^{13} \mathrm{C}$ NMR Spectra of Compound 3qa.

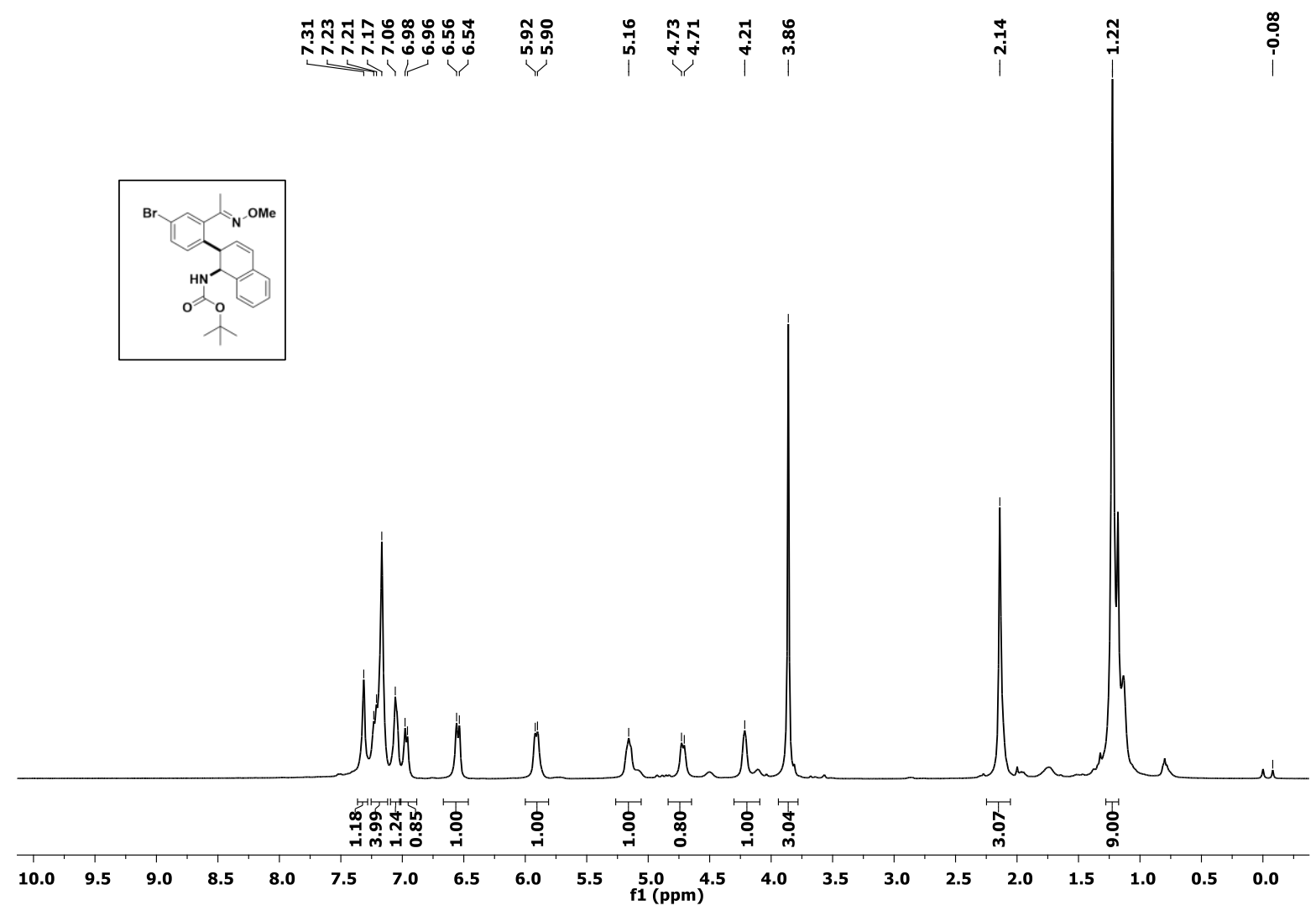

The NMR was taken at $50{ }^{\circ} \mathrm{C}$. The bumps range $5.0 \mathrm{ppm}$ and $4.4 \mathrm{ppm}$ were disappeared

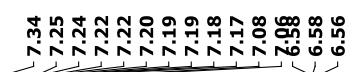

ไุ่

$\stackrel{\infty}{\sim \rightarrow n}$

$\stackrel{m}{\stackrel{m}{+}}$

$\stackrel{i}{\stackrel{4}{+}}$

$\left.\right|_{\substack{\infty \\ \infty}} ^{\substack{\infty \\ \prod}}$

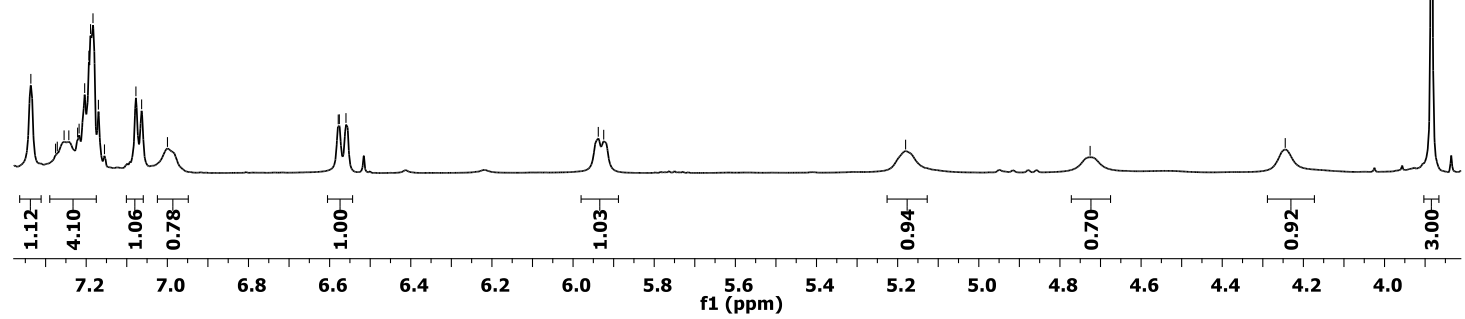




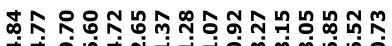

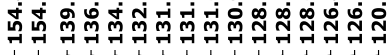

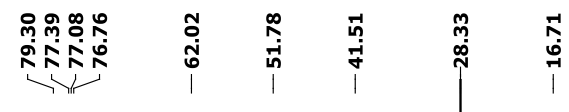
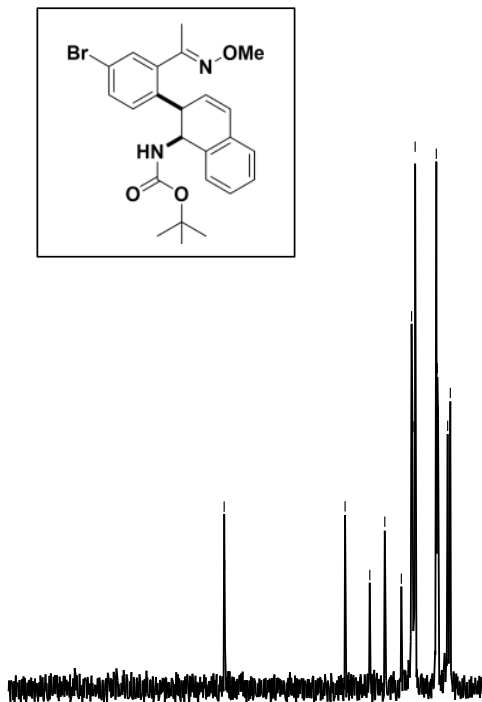

180

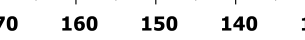

$\begin{array}{llll}130 & 120 & 110 & 100\end{array}$

$90 \underset{\text { f1 }}{90} 80$

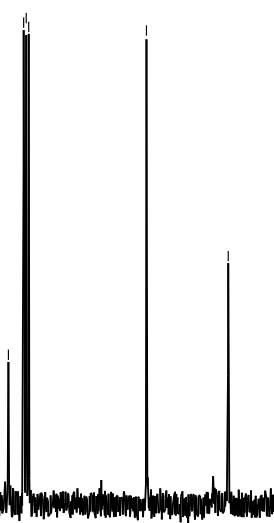

$\begin{array}{lllllll}50 & 40 & 30 & 20 & 10 & 0 & -10\end{array}$ 
${ }^{1} \mathrm{H}$ and ${ }^{13} \mathrm{C}$ NMR Spectra of Compound 3ra.

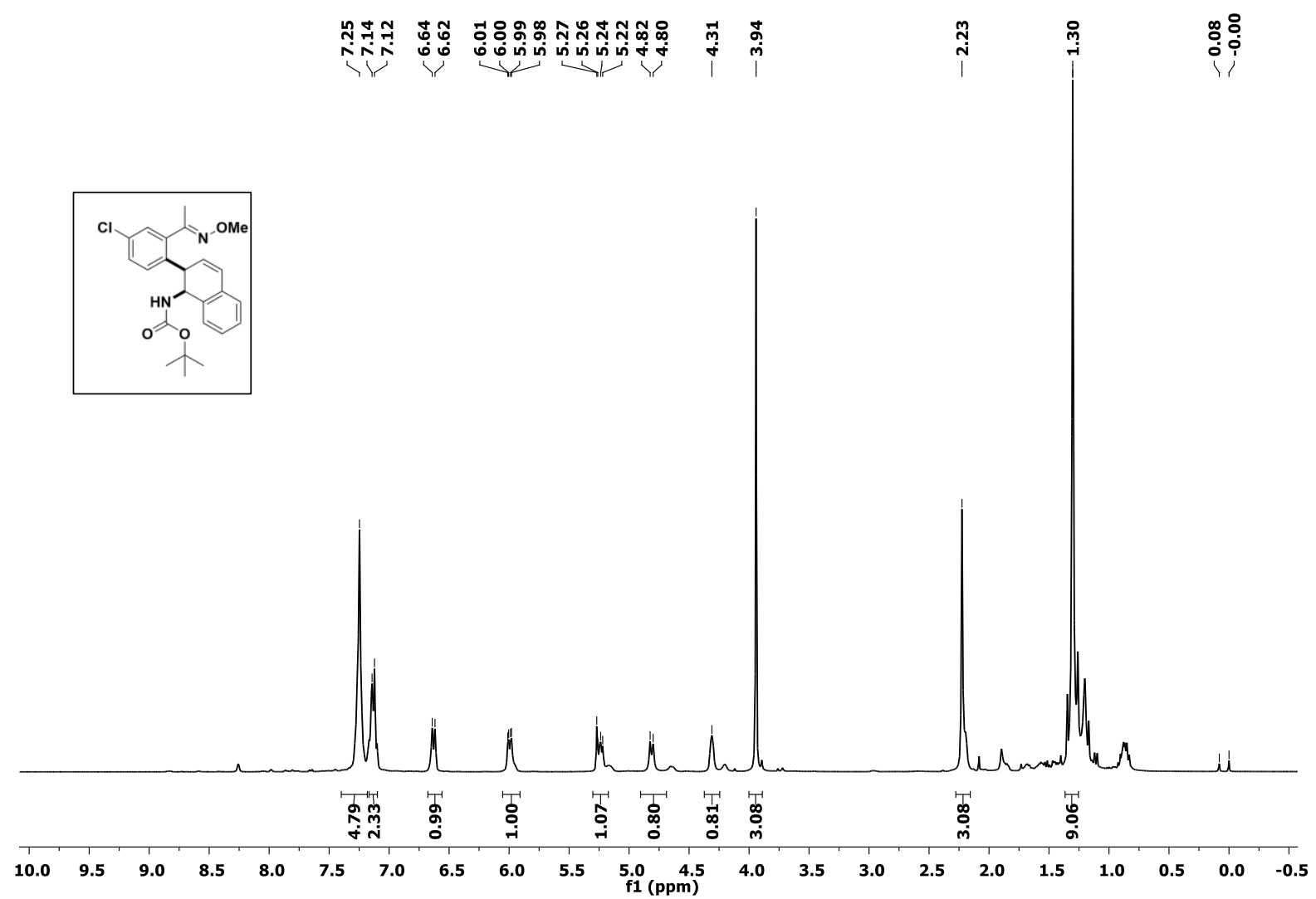

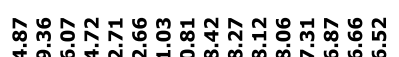

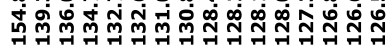

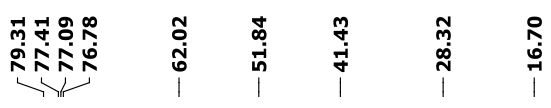
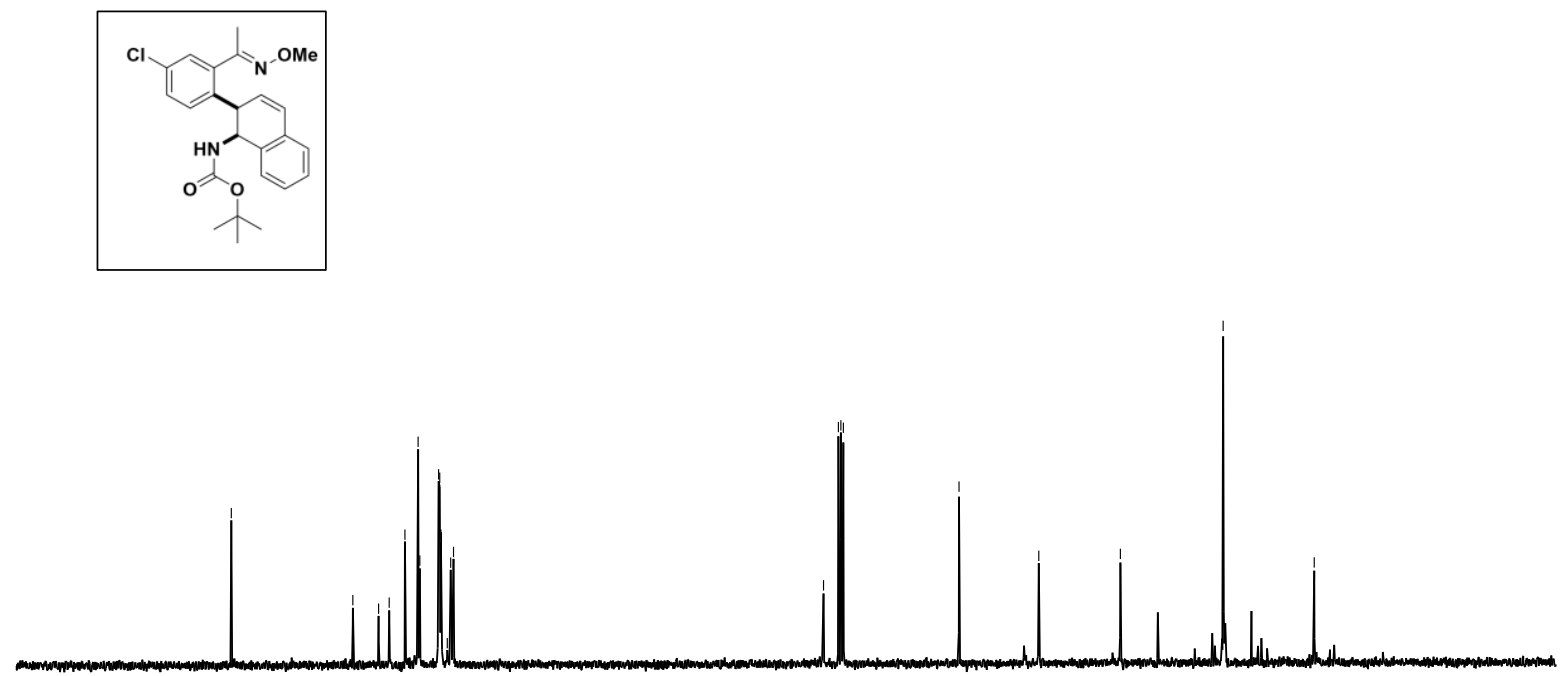

180

$\begin{array}{llllllllll}170 & 160 & 150 & 140 & 130 & 120 & 110 & 100 & 90 \begin{array}{r}80 \\ \text { f1 (ppm) }\end{array}\end{array}$

6050

40

$30 \quad 20$

$10 \quad-10$ 
${ }^{1} \mathrm{H}$ and ${ }^{13} \mathrm{C}$ NMR Spectra of Compound 3sa.

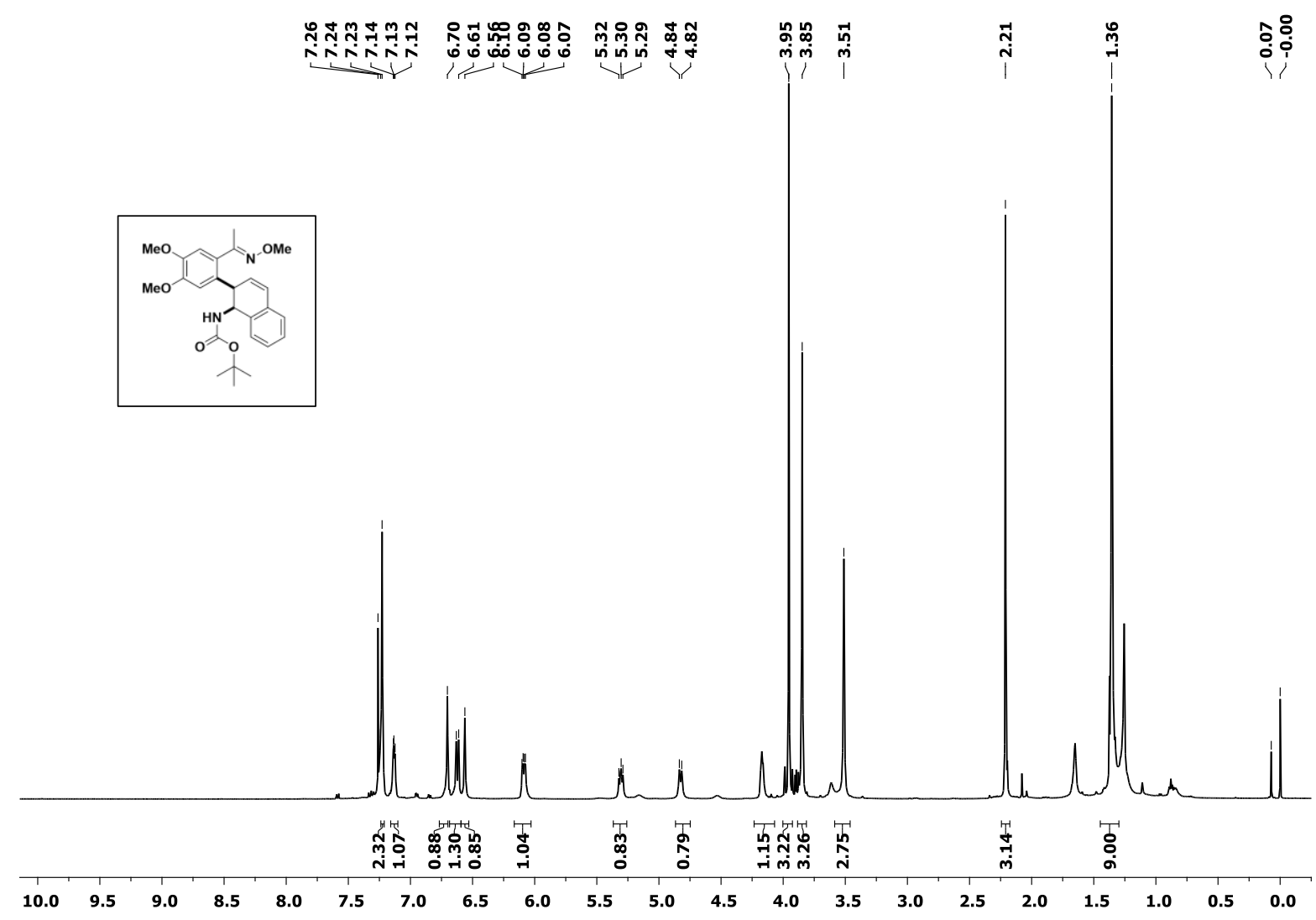

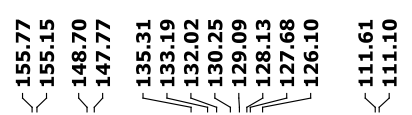

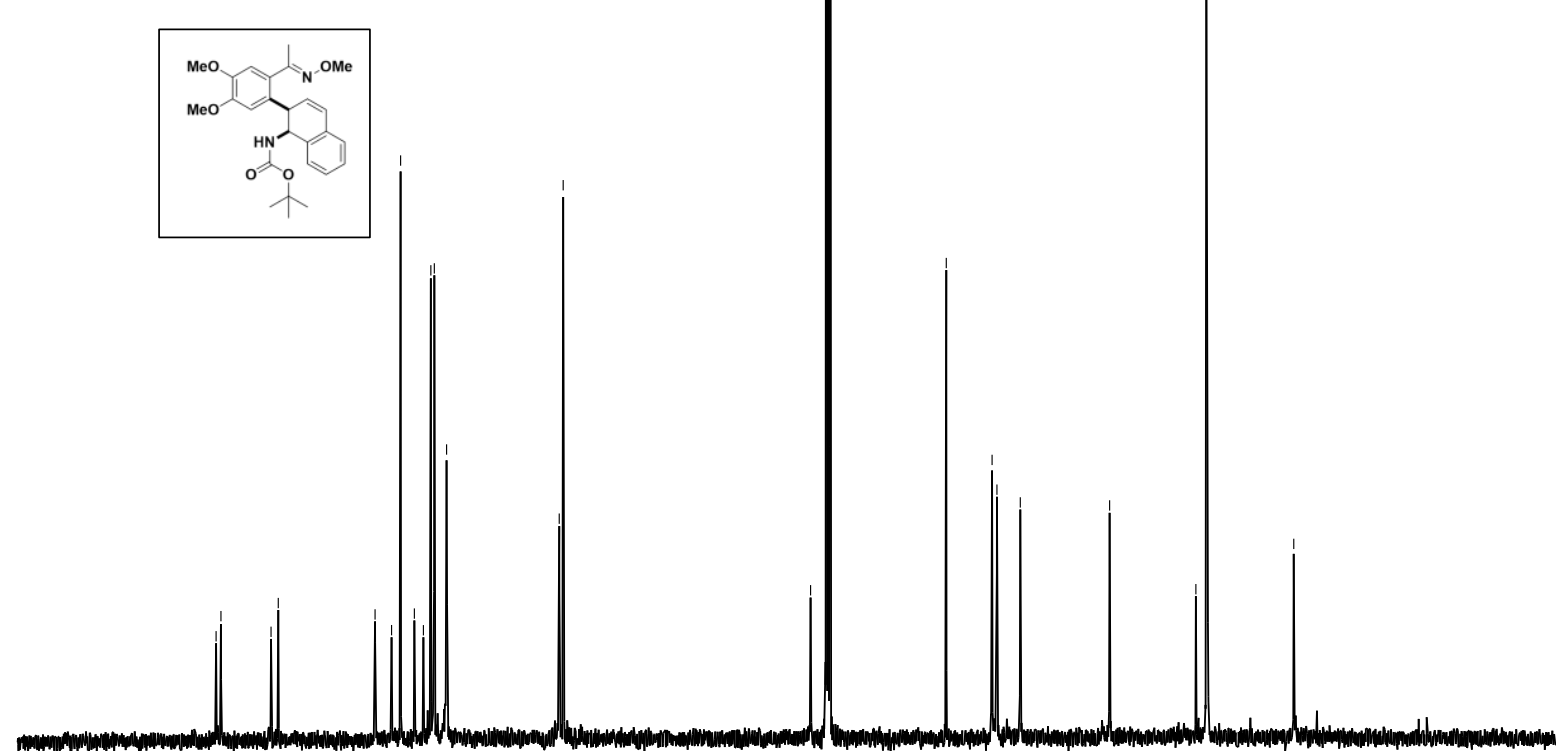

180

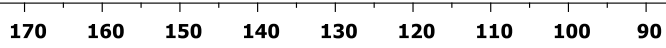

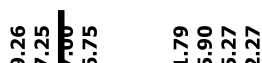

ก

S35 
DEPT (135) NMR Spectrum of Compound 3sa.

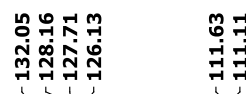

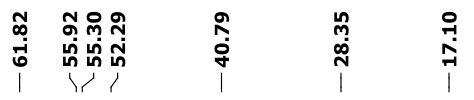
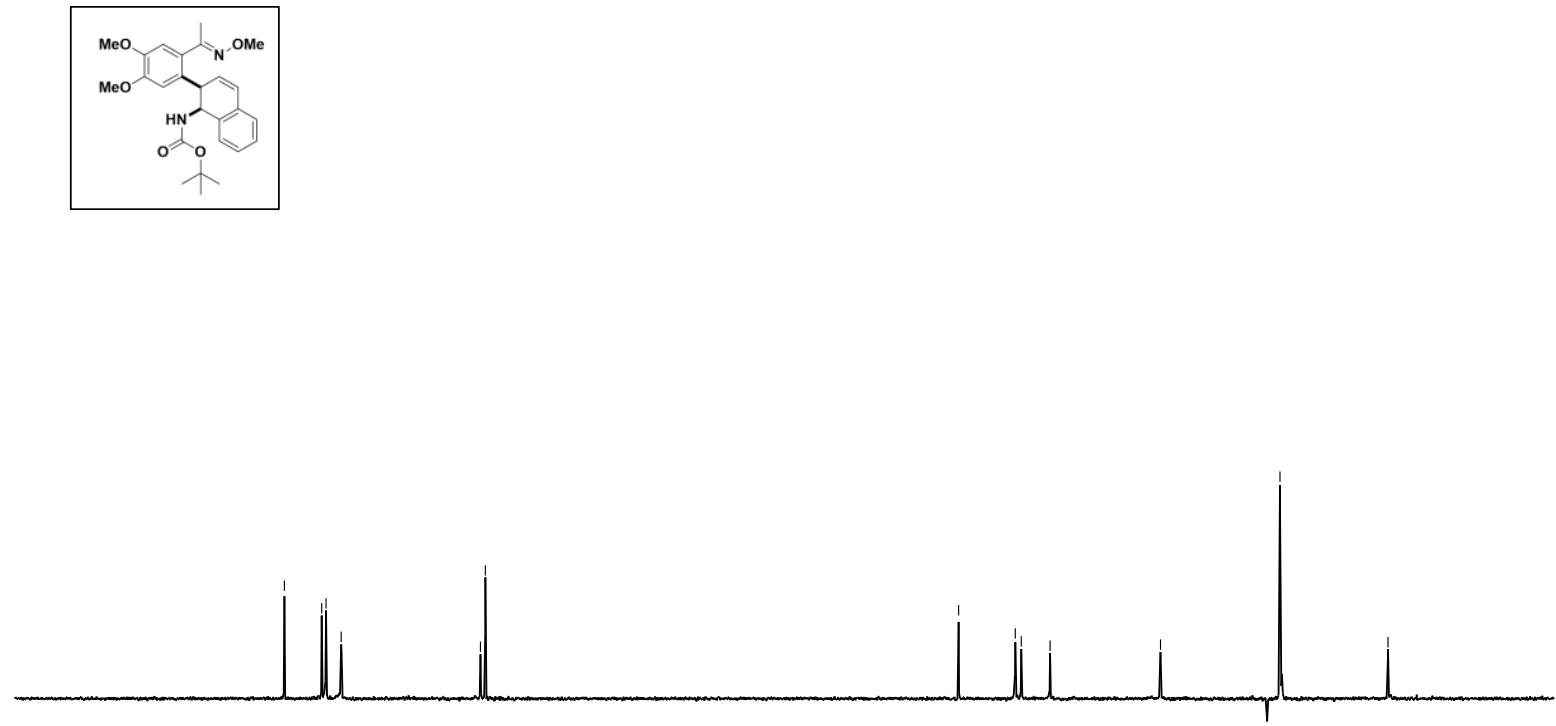

$\begin{array}{lllllllllllllllll}160 & 150 & 140 & 130 & 120 & 110 & 100 & 90 & \underset{f 1}{8(\mathrm{ppm})} & 70 & 60 & 50 & 40 & 30 & 20 & 10 & 0\end{array}$ 
${ }^{1} \mathrm{H}$ and ${ }^{13} \mathrm{C}$ NMR Spectra of Compound 3 ta.

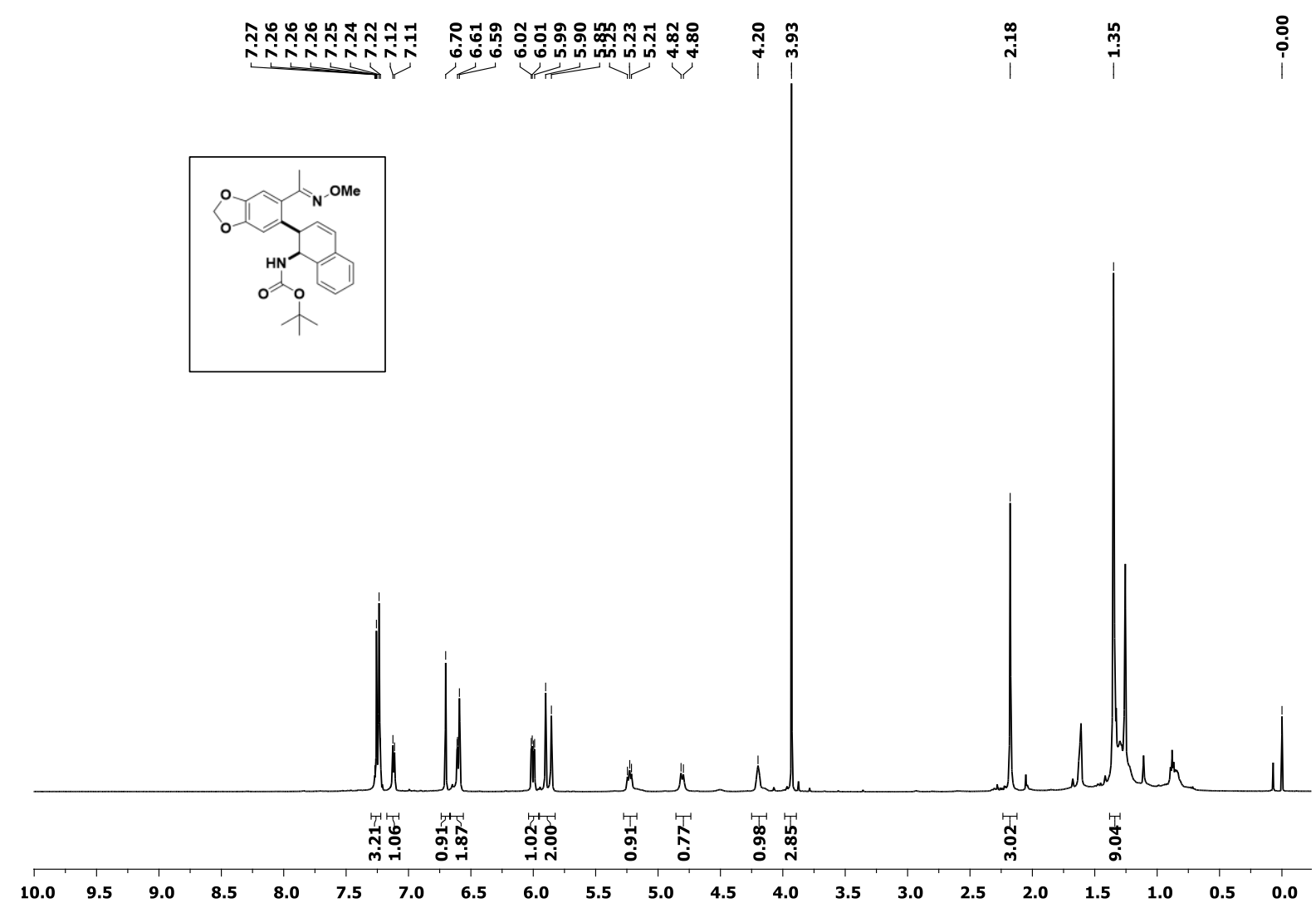

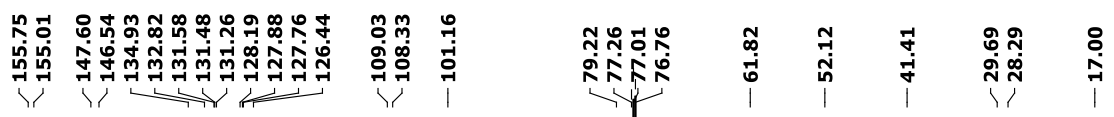

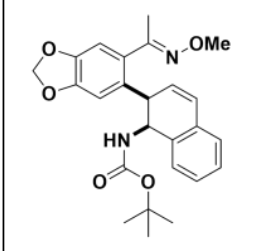

180

$\begin{array}{lllllllllll}180 & 170 & 160 & 150 & 140 & 130 & 120 & 110 & 100 & 90 & 80\end{array}$ 
DEPT (135) NMR Spectrum of Compound 3ta.

ت
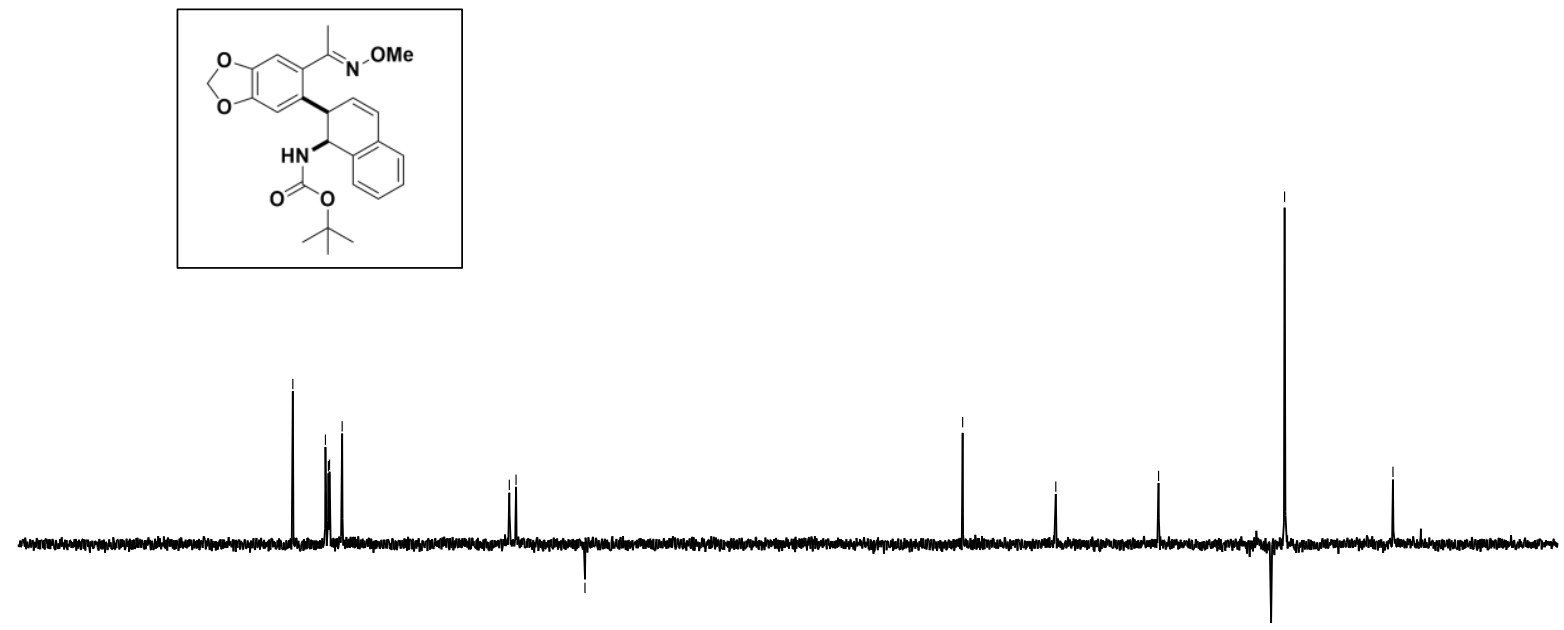

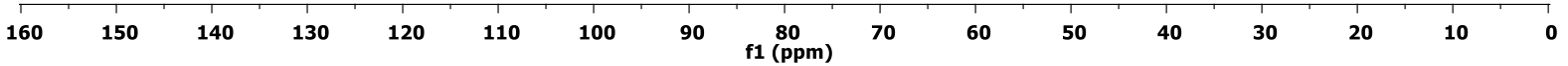


${ }^{1} \mathrm{H}$ and ${ }^{13} \mathrm{C}$ NMR Spectra of Compound 3ua.

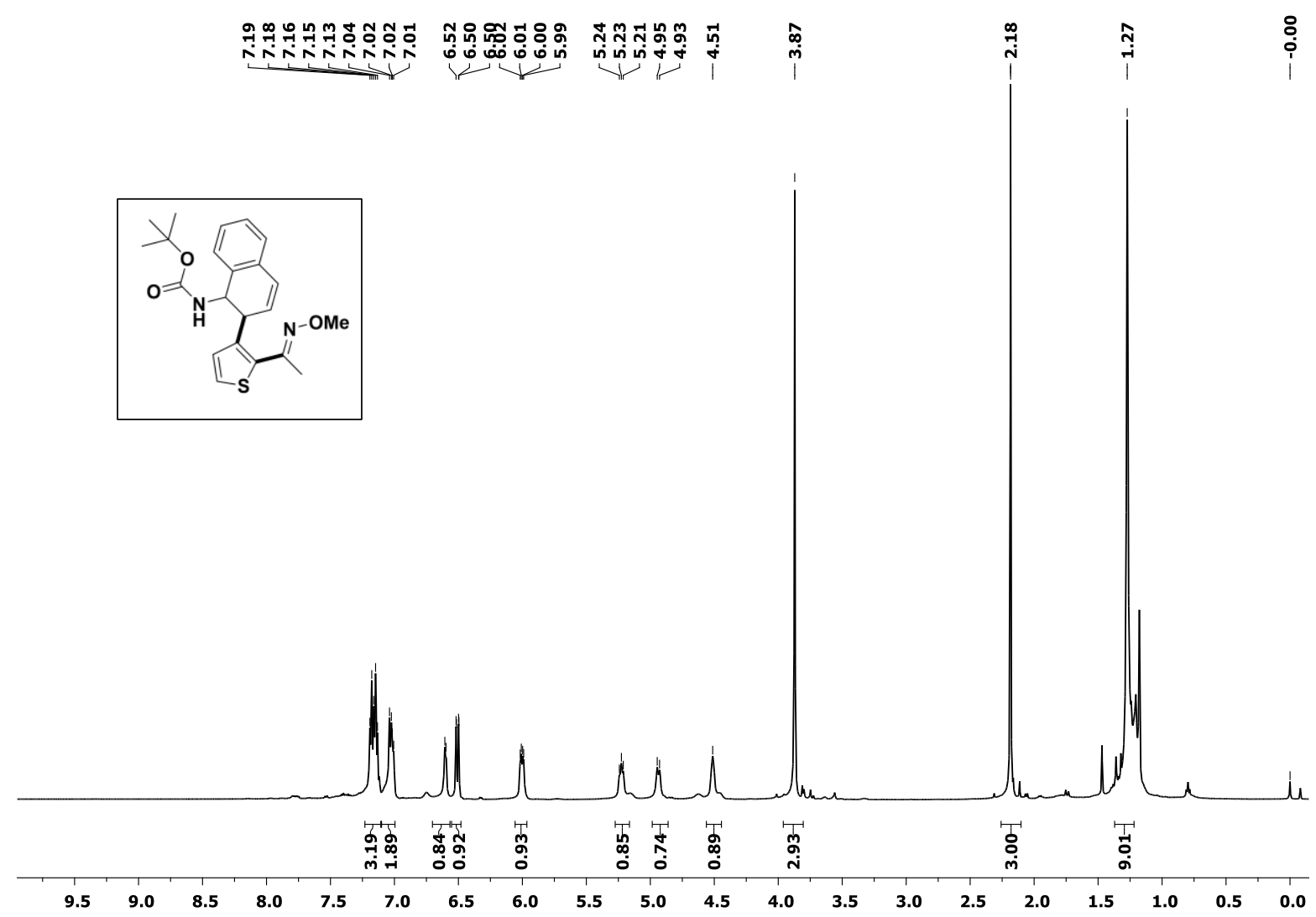

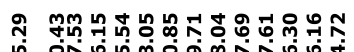

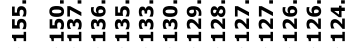

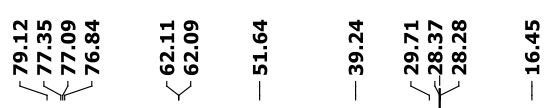

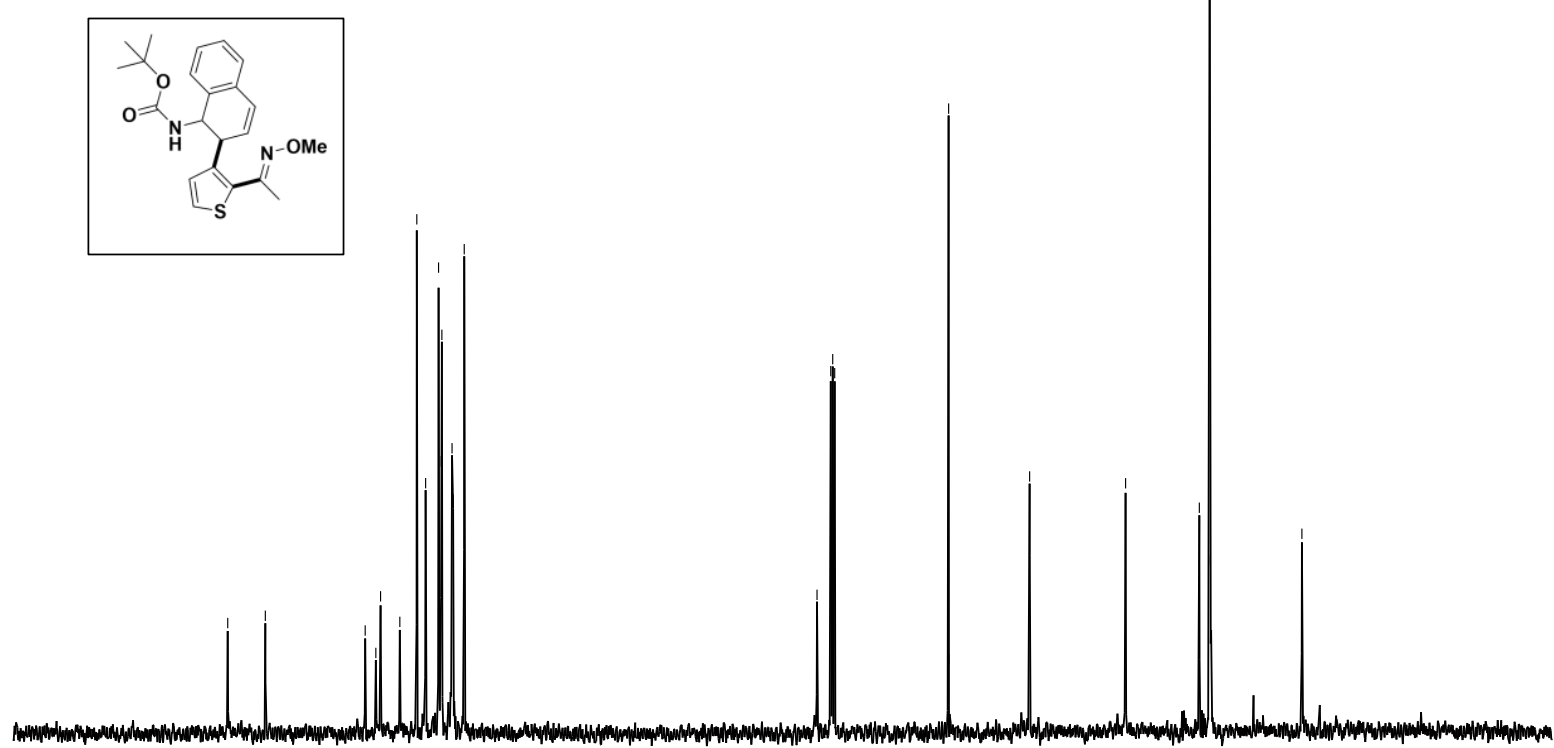

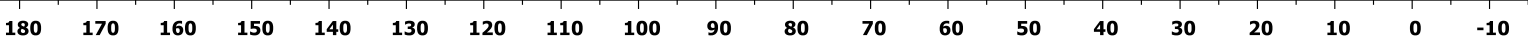


DEPT (135) NMR Spectrum of Compound 3ua.

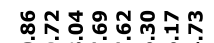

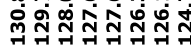

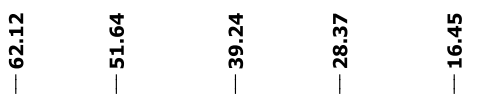

-OMe

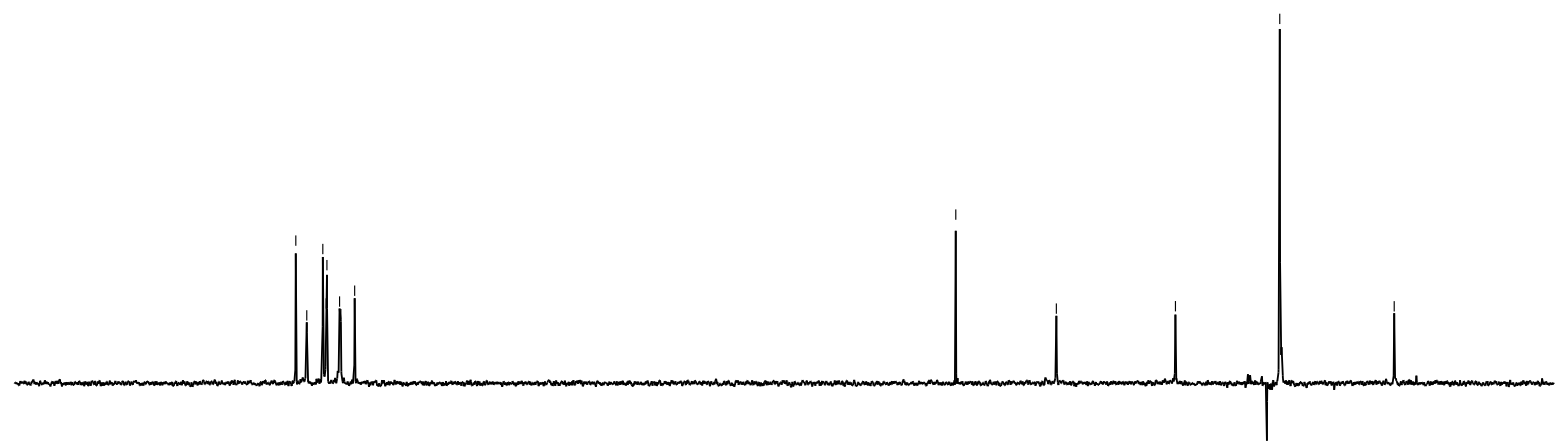

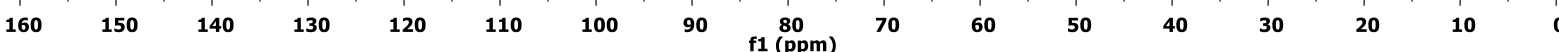


${ }^{1} \mathrm{H}$ and ${ }^{13} \mathrm{C}$ NMR Spectra of Compound 3eb.
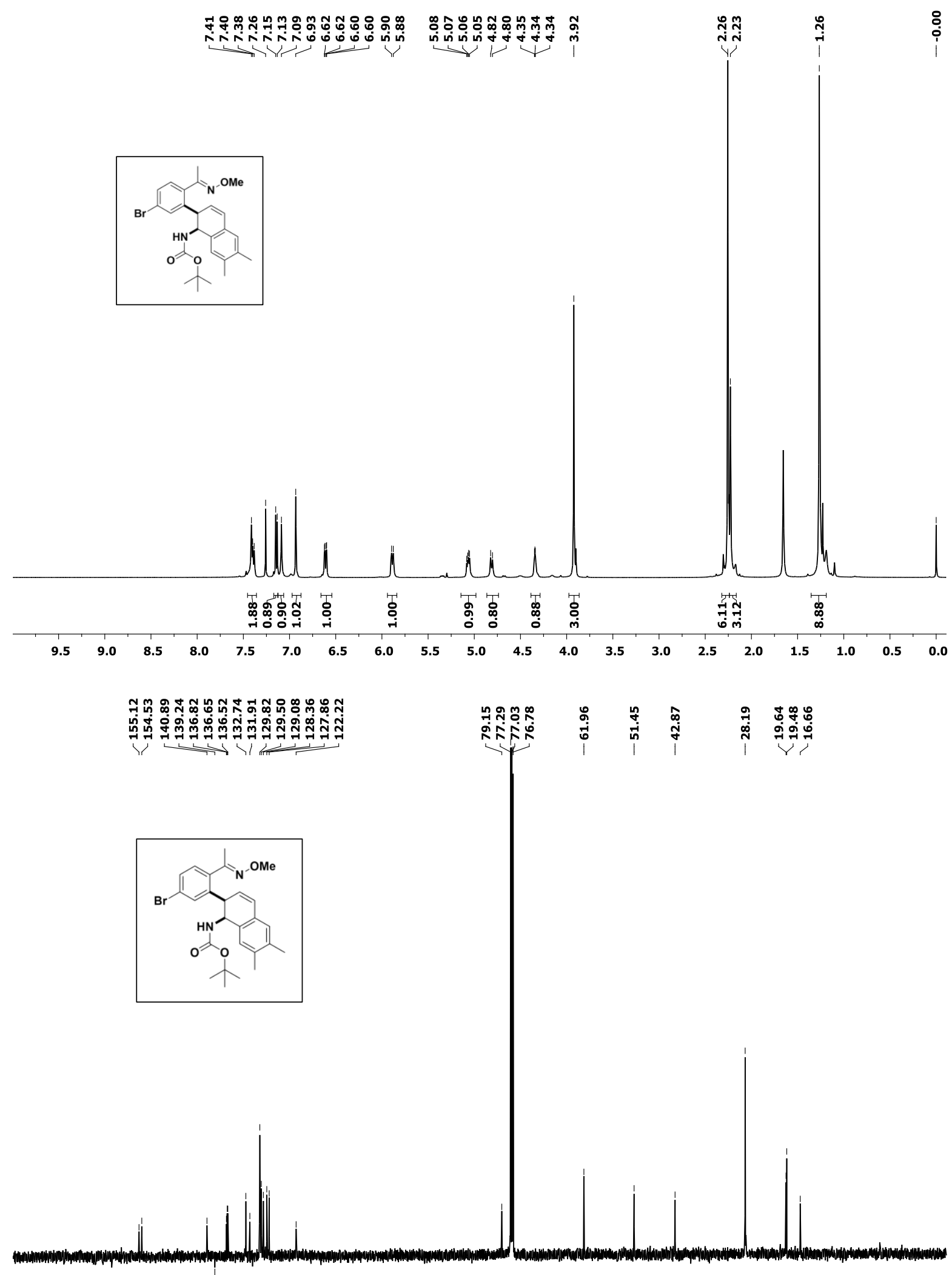

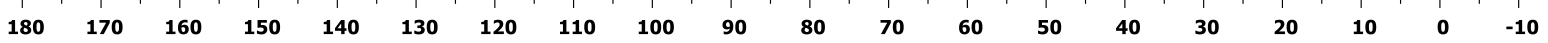


DEPT (135) NMR Spectrum of Compound 3eb.

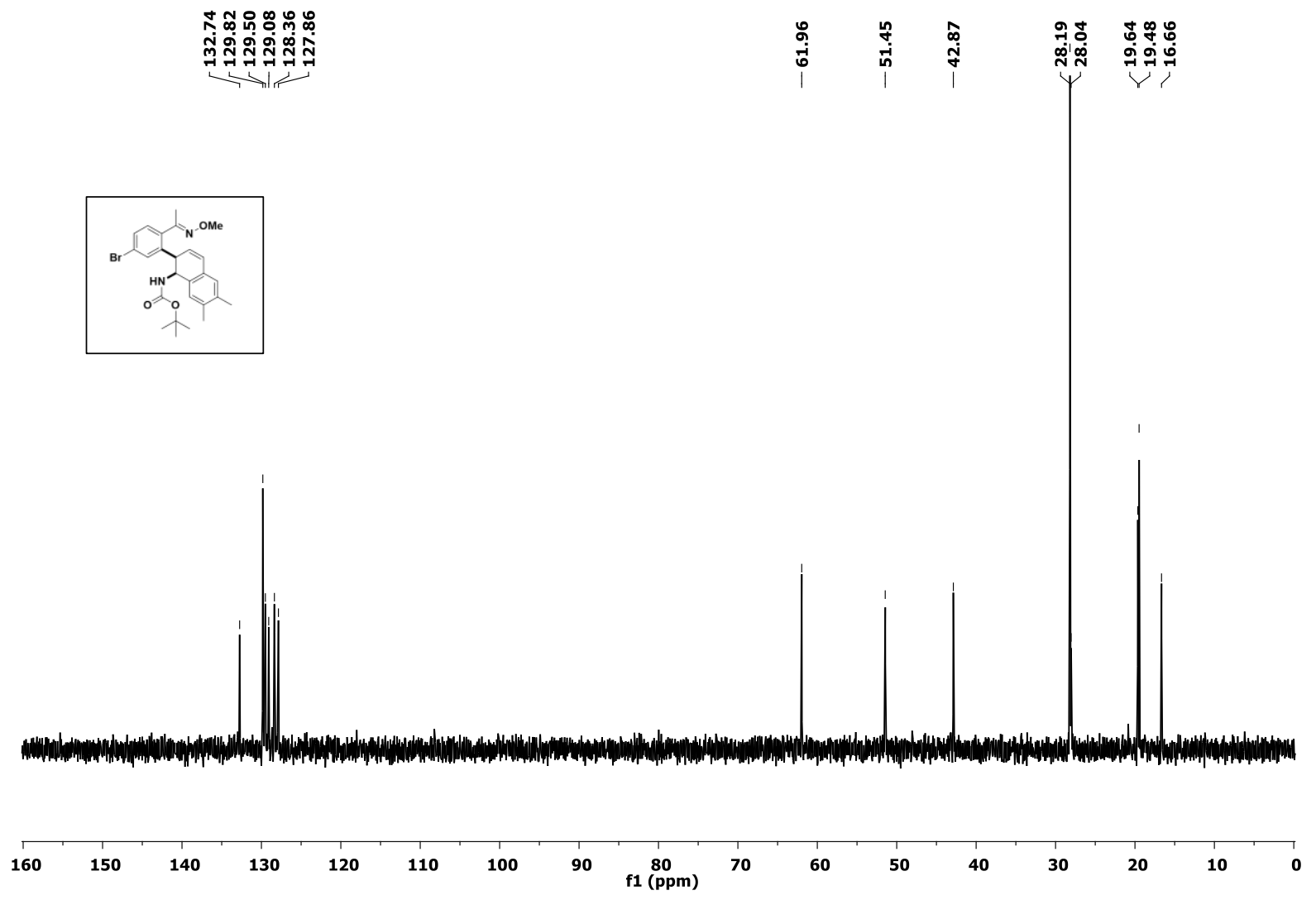


${ }^{1} \mathrm{H}$ and ${ }^{13} \mathrm{C}$ NMR Spectra of Compound 3cc.
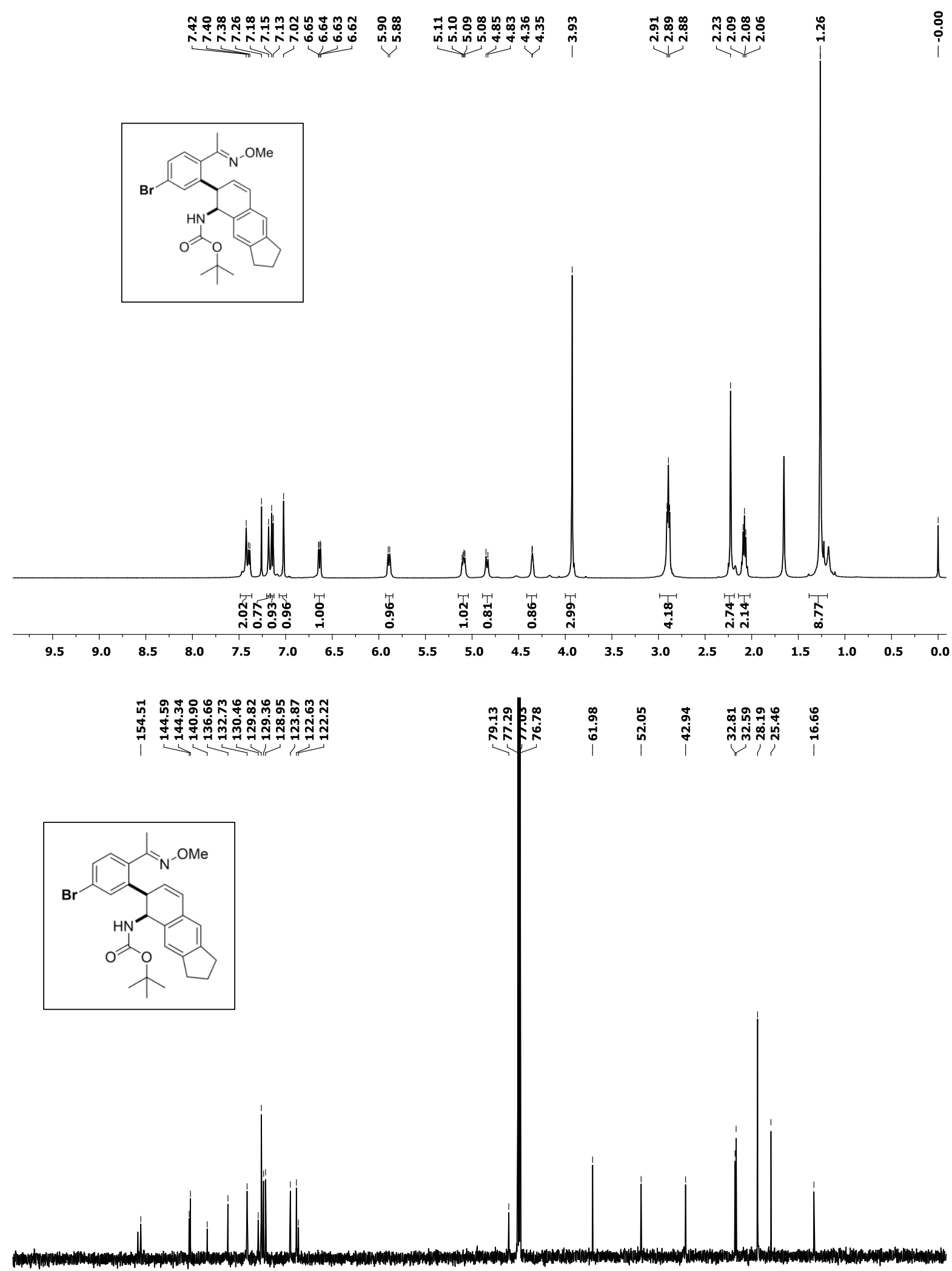

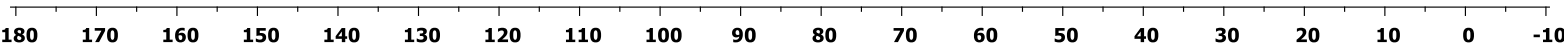


DEPT (135) NMR Spectrum of Compound 3cc.

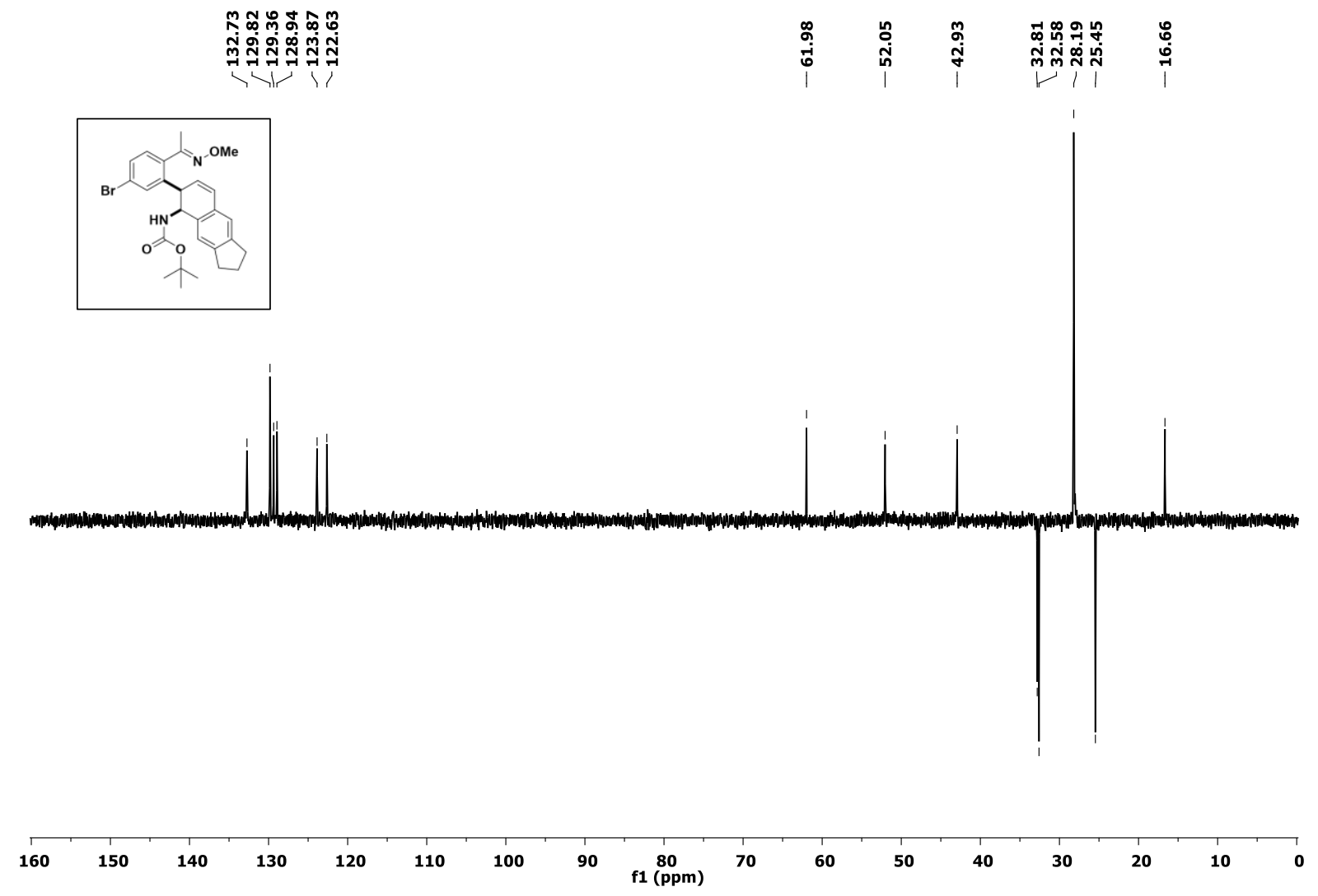


${ }^{1} \mathrm{H}$ and ${ }^{13} \mathrm{C}$ NMR Spectra of Compound 3ec.

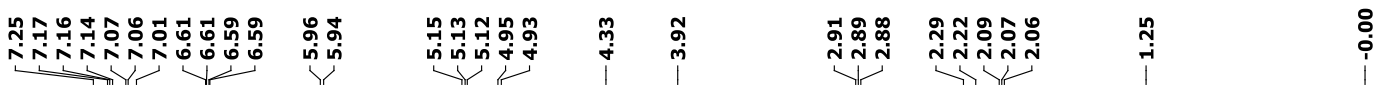

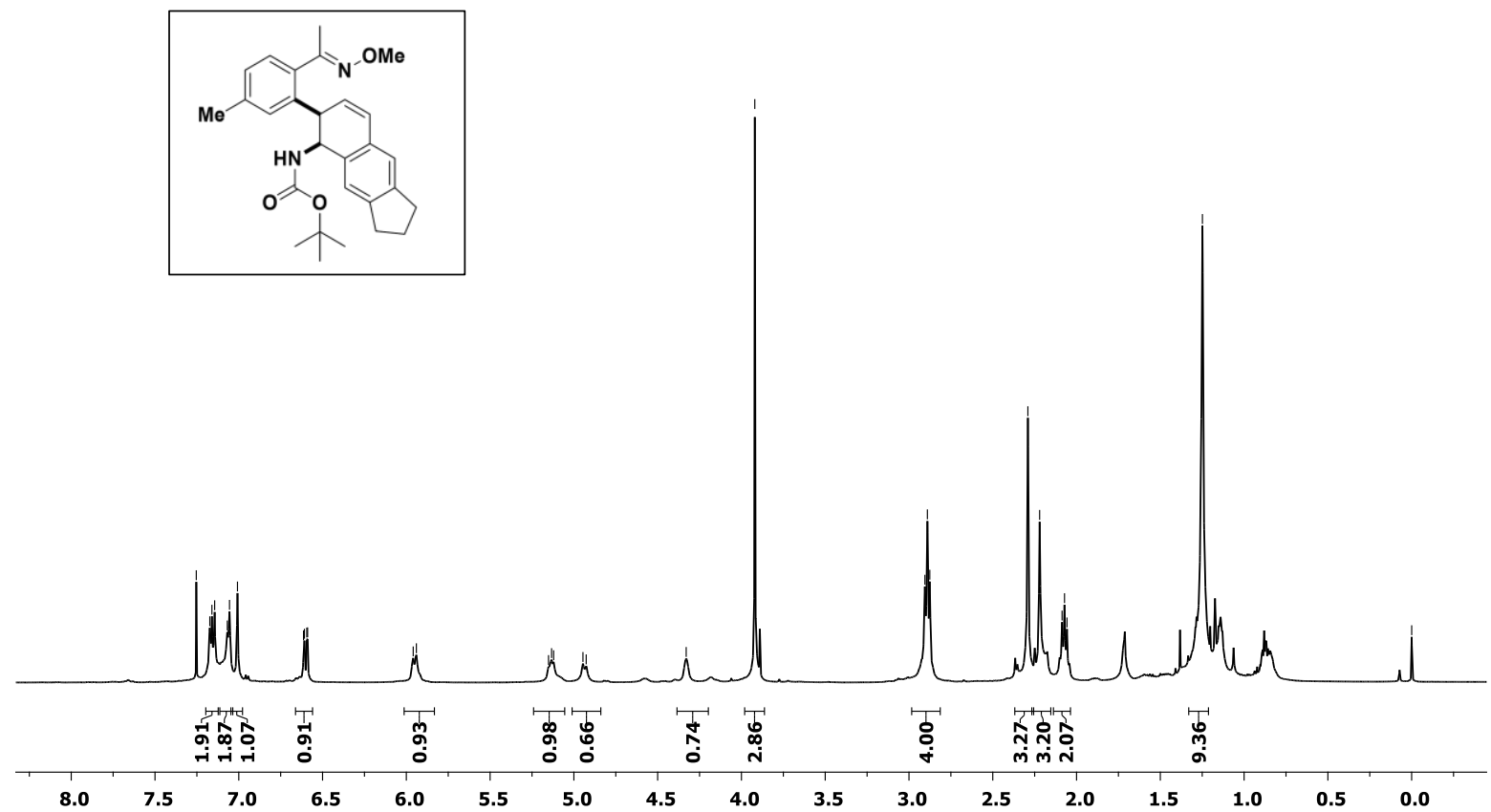

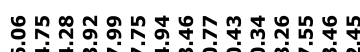

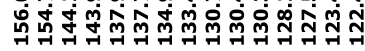

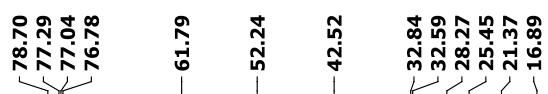

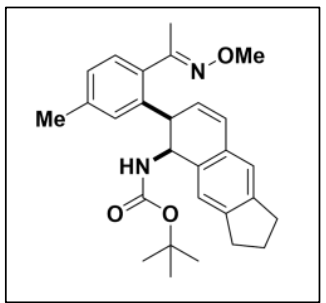

180

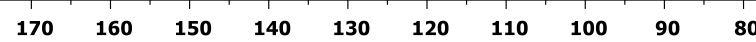

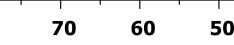

$\begin{array}{llllll}40 & 30 & 20 & 10 & 0 & -10\end{array}$ 
${ }^{1} \mathrm{H}$ and ${ }^{13} \mathrm{C}$ NMR Spectra of Compound 3cd.

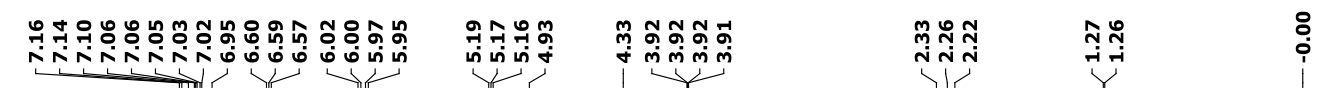
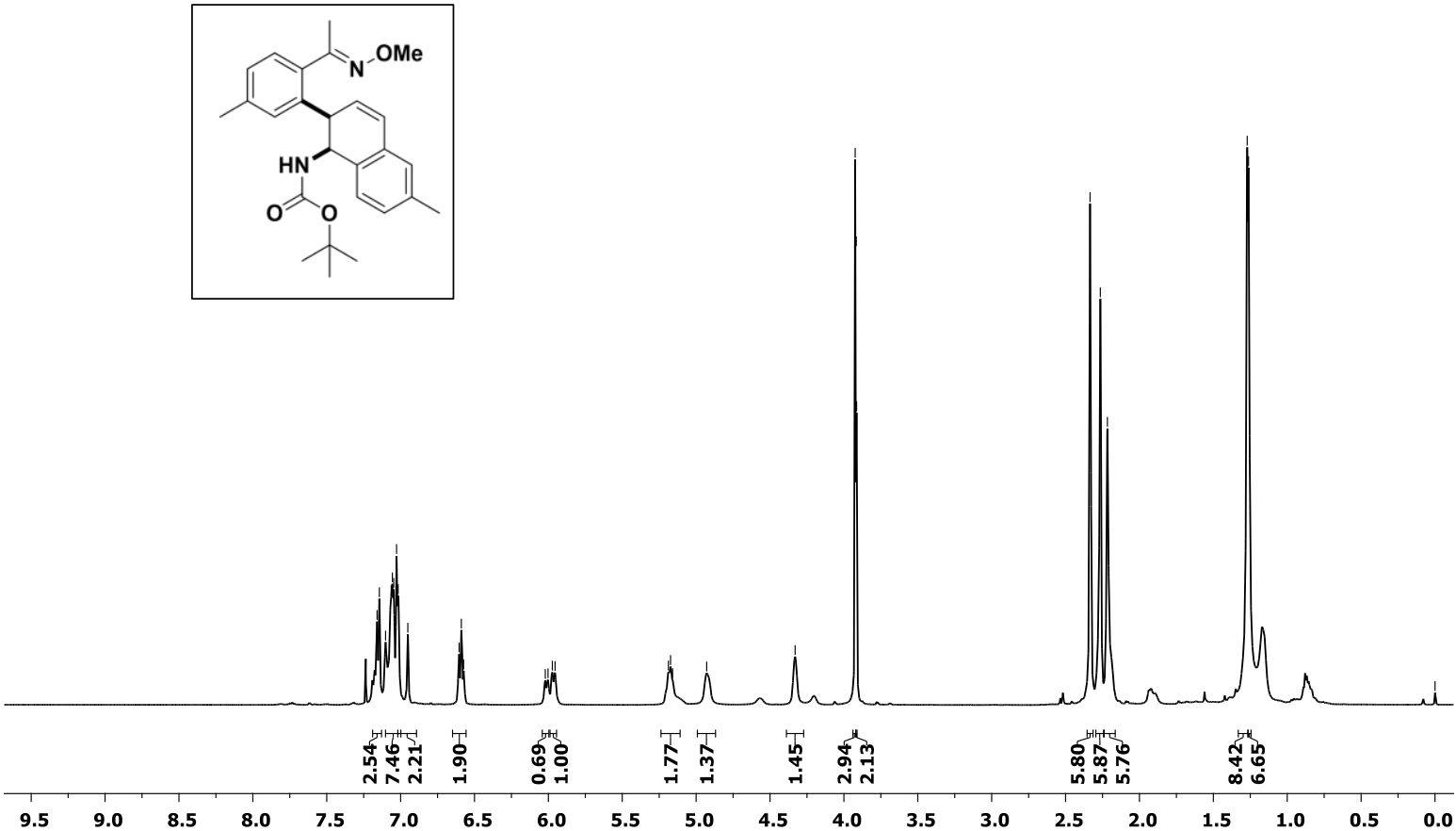

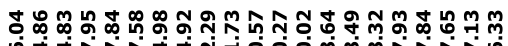

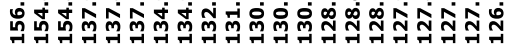

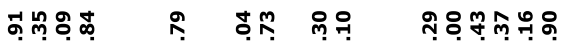

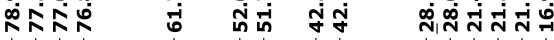

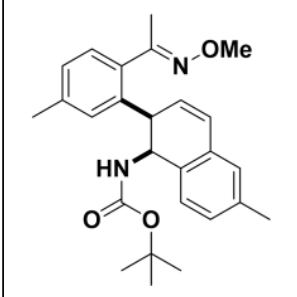

180

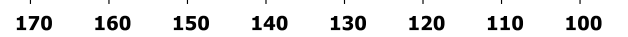

$\begin{array}{lllll}90 & 80 & 70 & 60 & 50\end{array}$

40

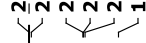


DEPT (135) NMR Spectrum of Compound 3cd.

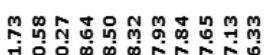

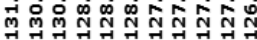

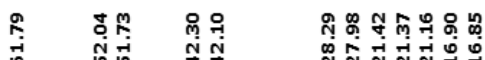

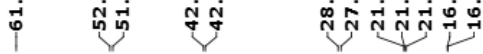
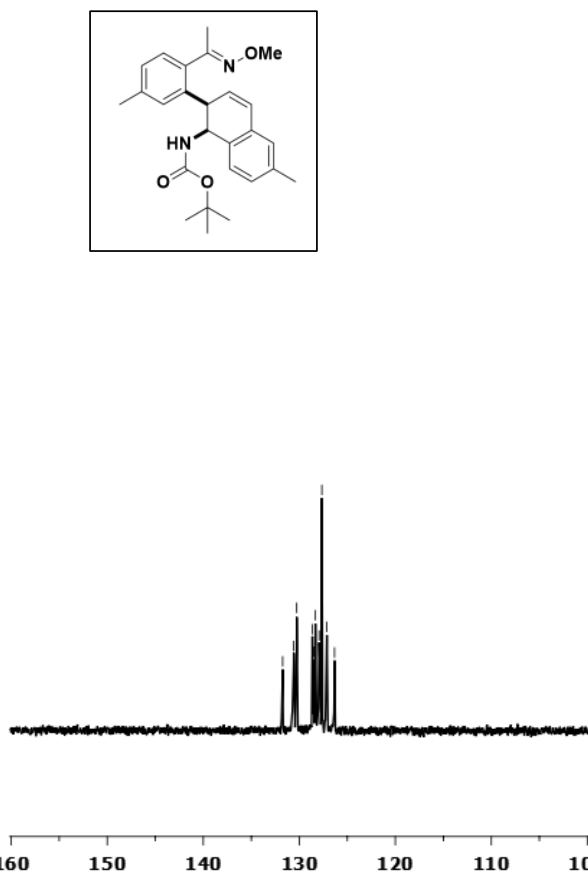

0090

$\stackrel{80}{\text { f1 }}$

605

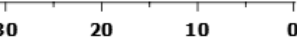


${ }^{1} \mathrm{H}$ and ${ }^{13} \mathrm{C}$ NMR Spectra of Compound 3ee.

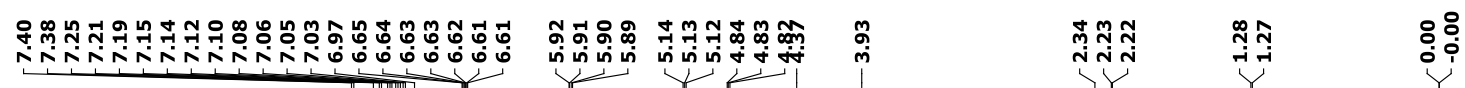
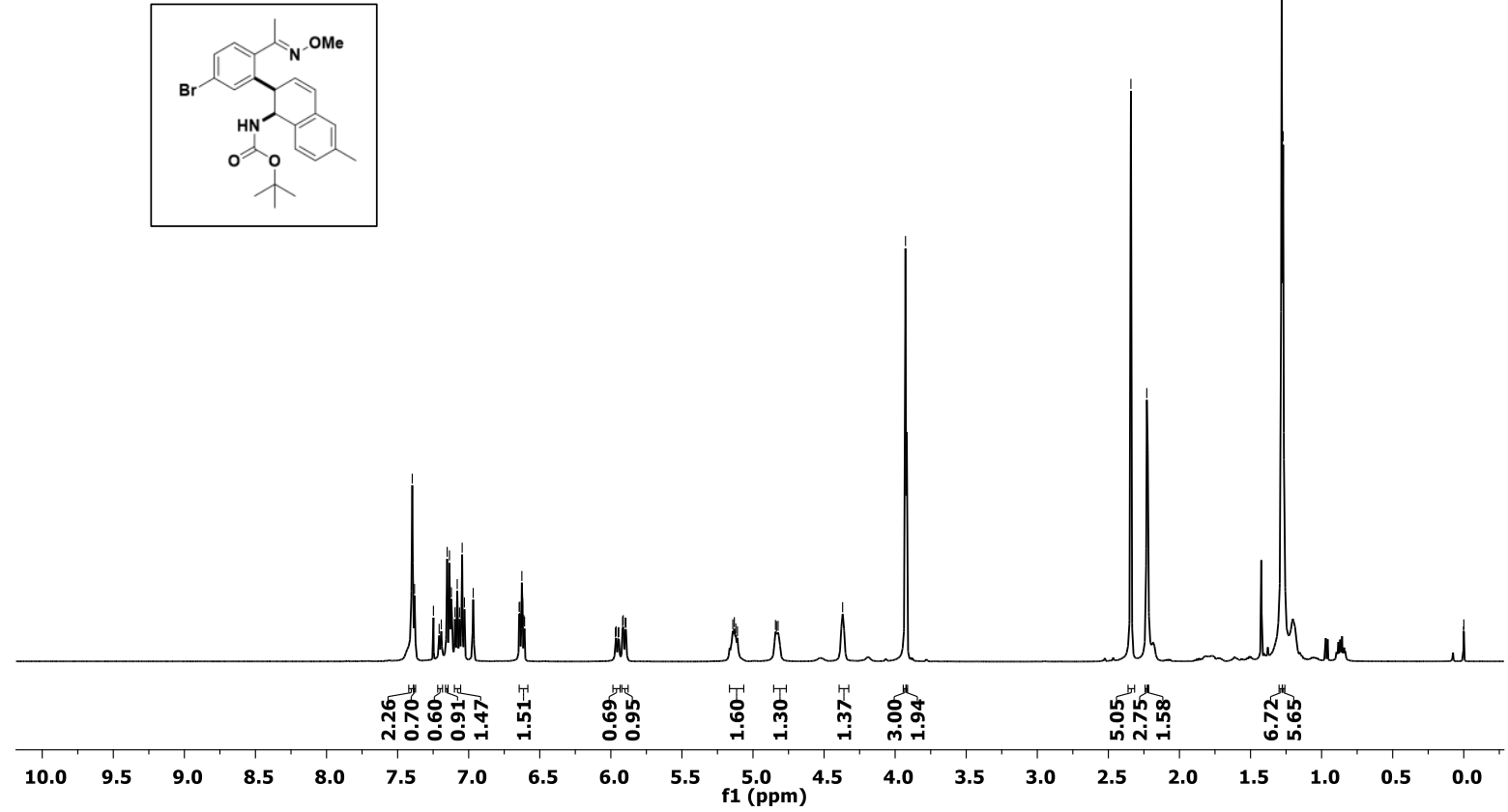

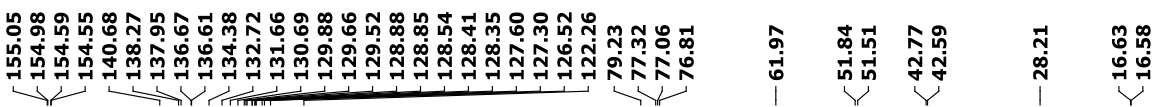
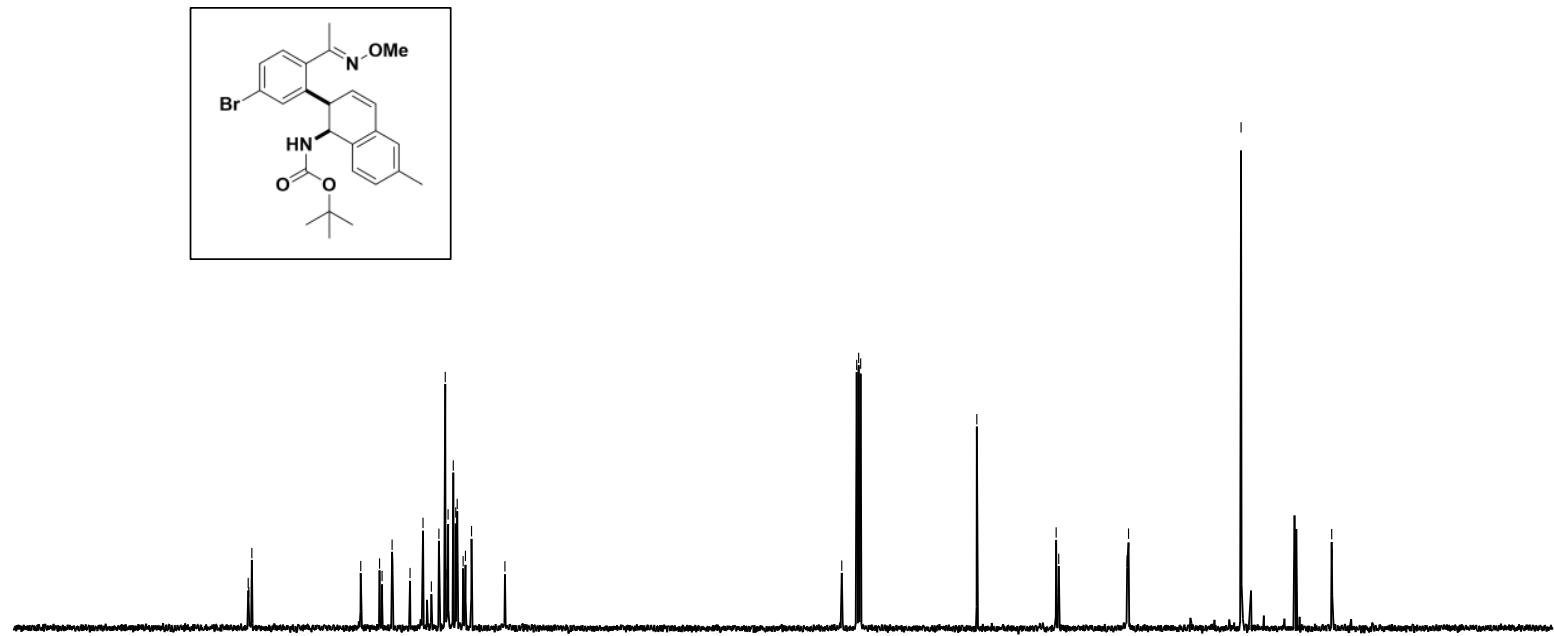

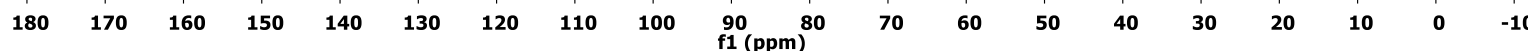


${ }^{1} \mathrm{H}$ and ${ }^{13} \mathrm{C}$ NMR Spectra of Compound 3ae.
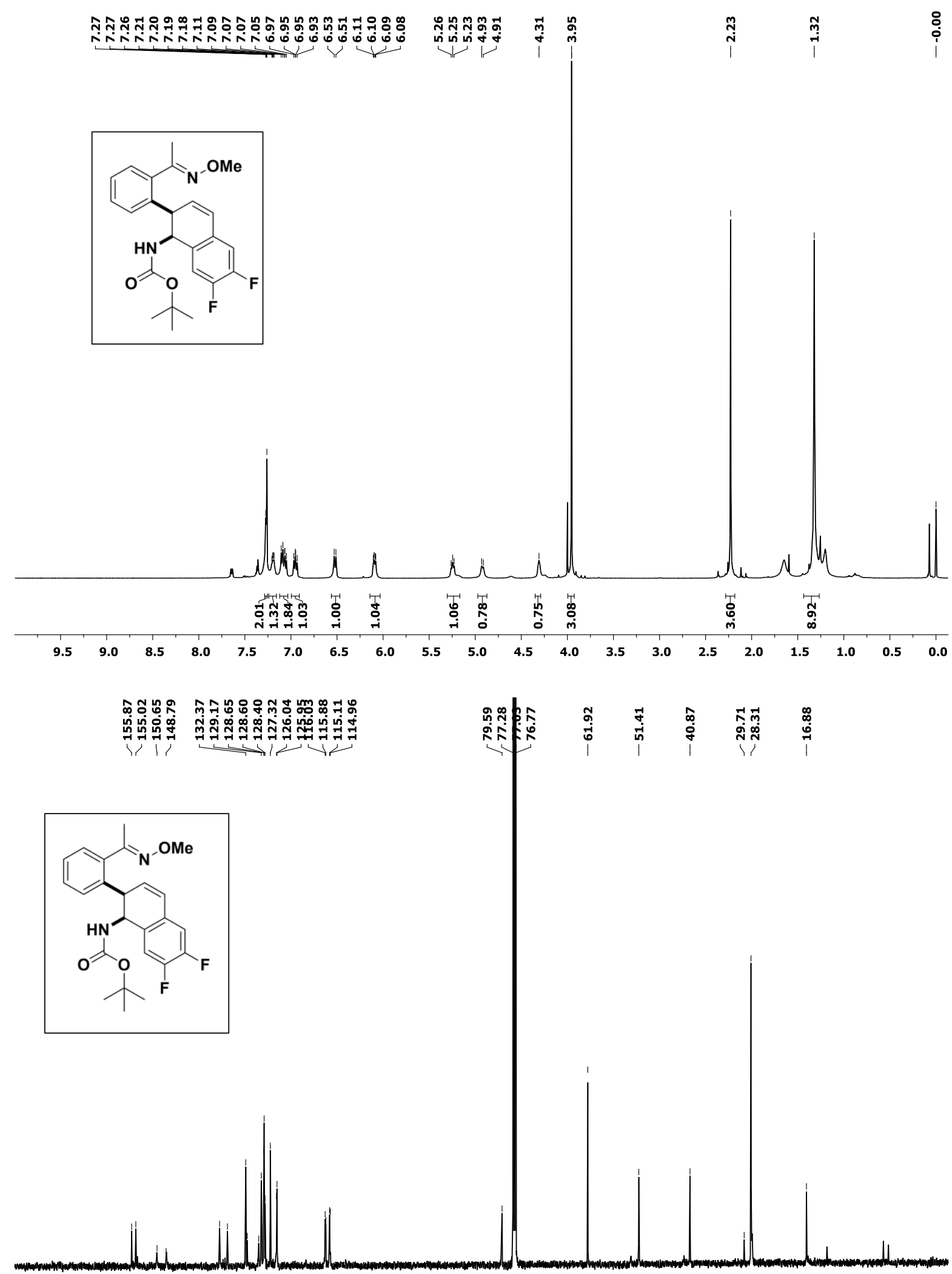

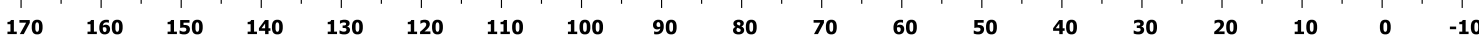


DEPT (135) NMR Spectrum of Compound 3ae.

กิ

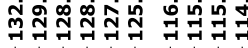

가난
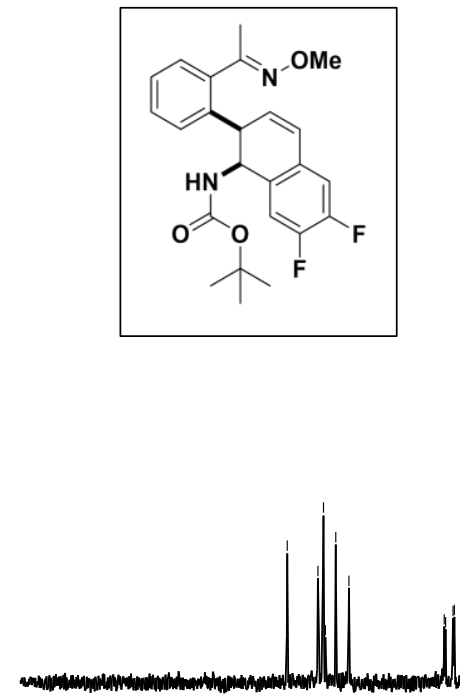

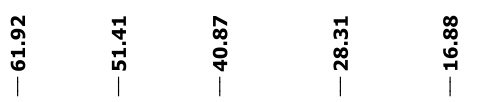

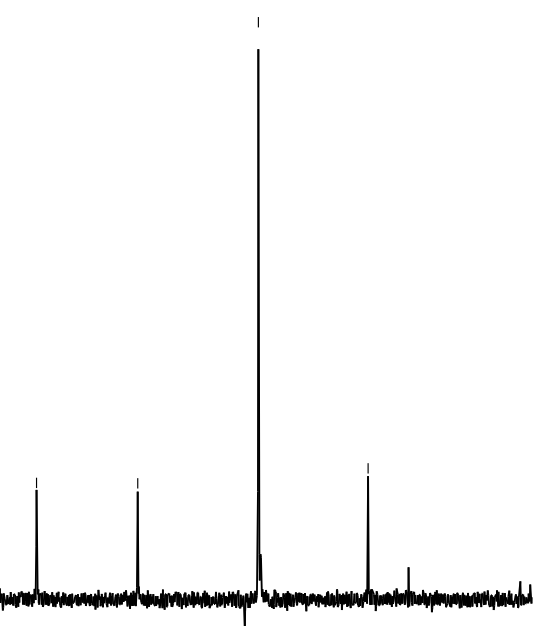


${ }^{1} \mathrm{H}$ and ${ }^{13} \mathrm{C}$ NMR Spectra of Compound 3ec.
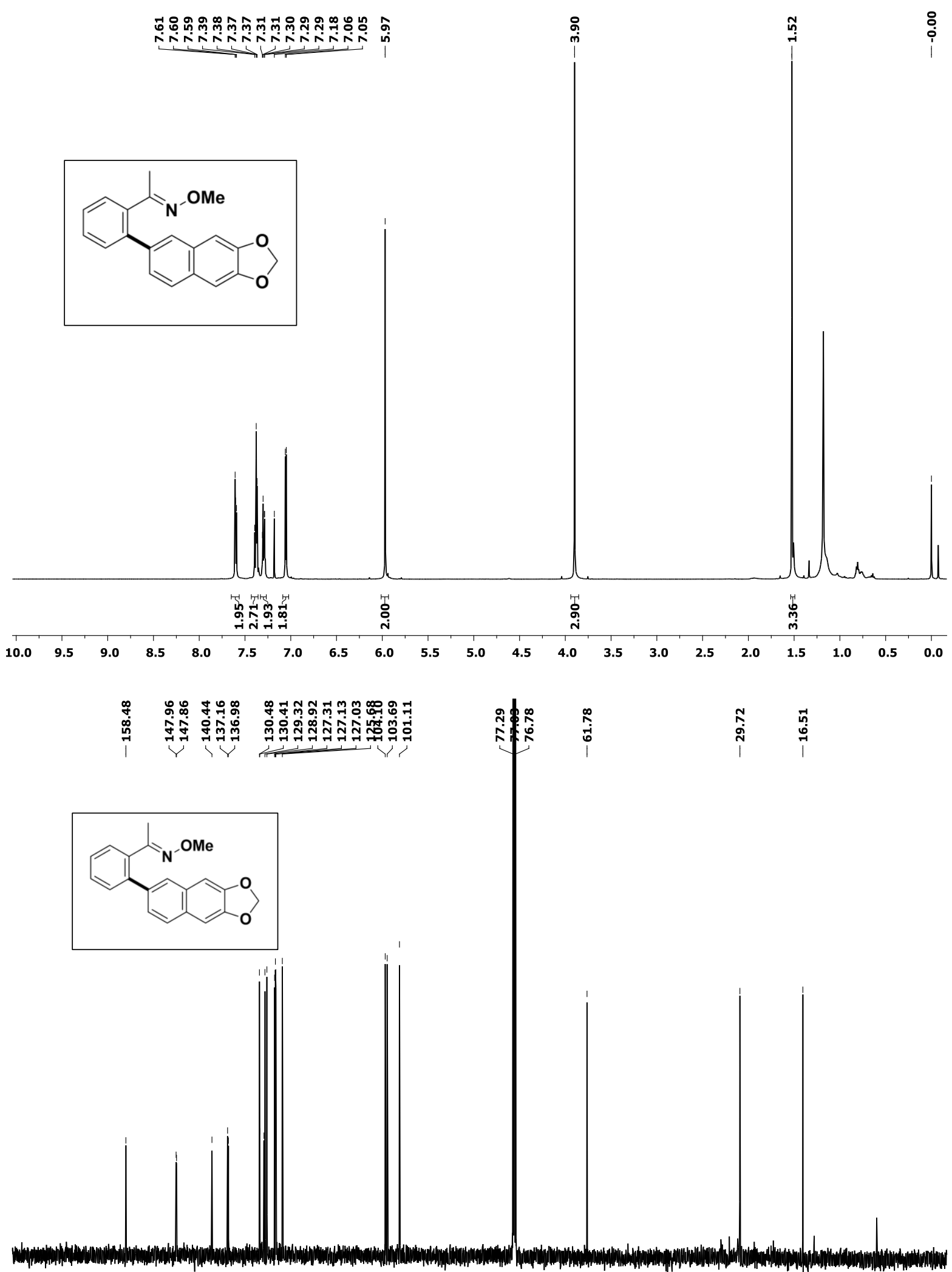

$\begin{array}{lllllllllllllllllllllll}180 & 170 & 160 & 150 & 140 & 130 & 120 & 110 & 100 & 90 & 80 & 70 & 60 & 50 & 40 & 30 & 20 & 10 & 0 & -10\end{array}$ 
DEPT (135) NMR Spectrum of Compound 3ec.

वำ

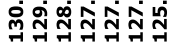

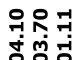

유음

$\stackrel{\infty}{\stackrel{0}{0}}$

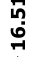
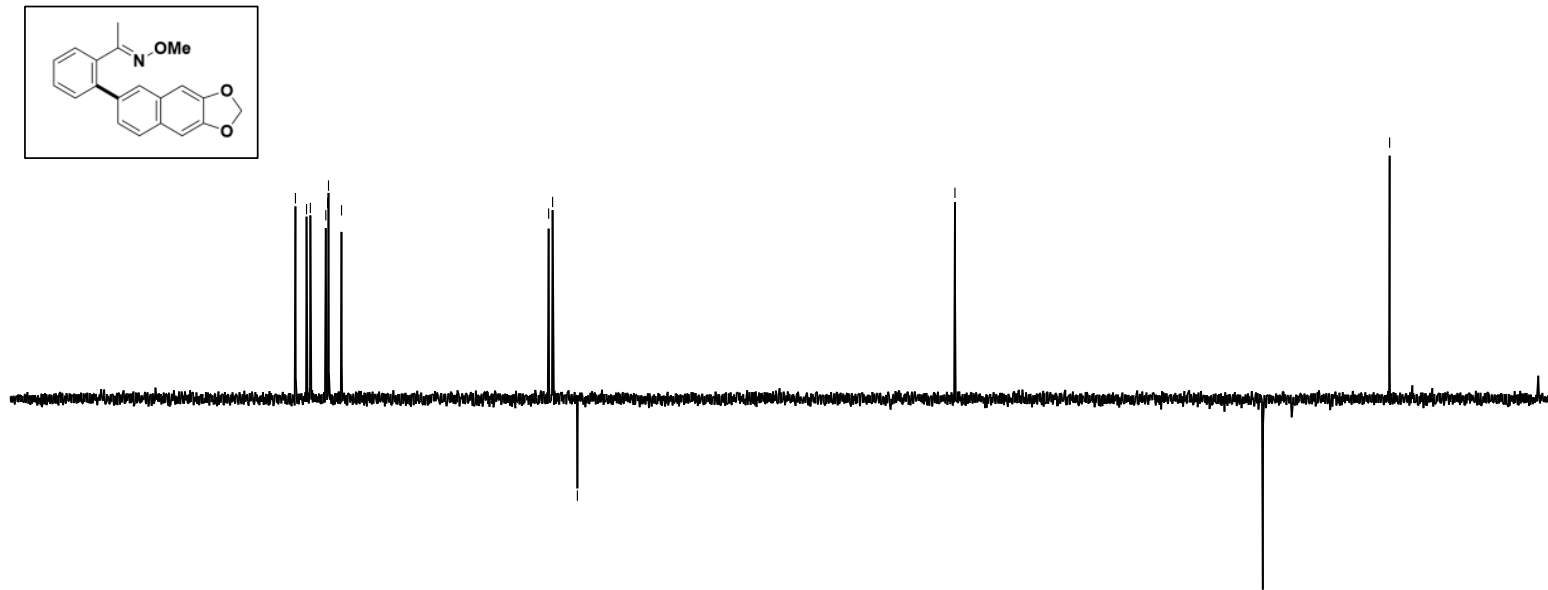

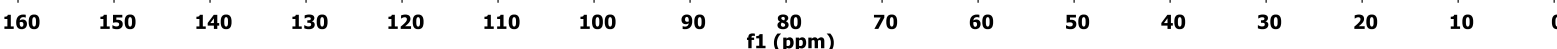


${ }^{1} \mathrm{H}$ and ${ }^{13} \mathrm{C}$ NMR Spectra of Compound $4 \mathbf{a}$.

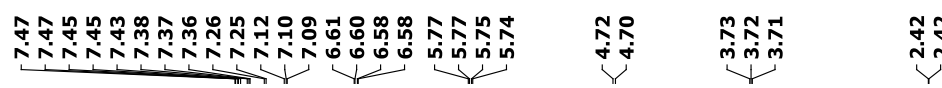
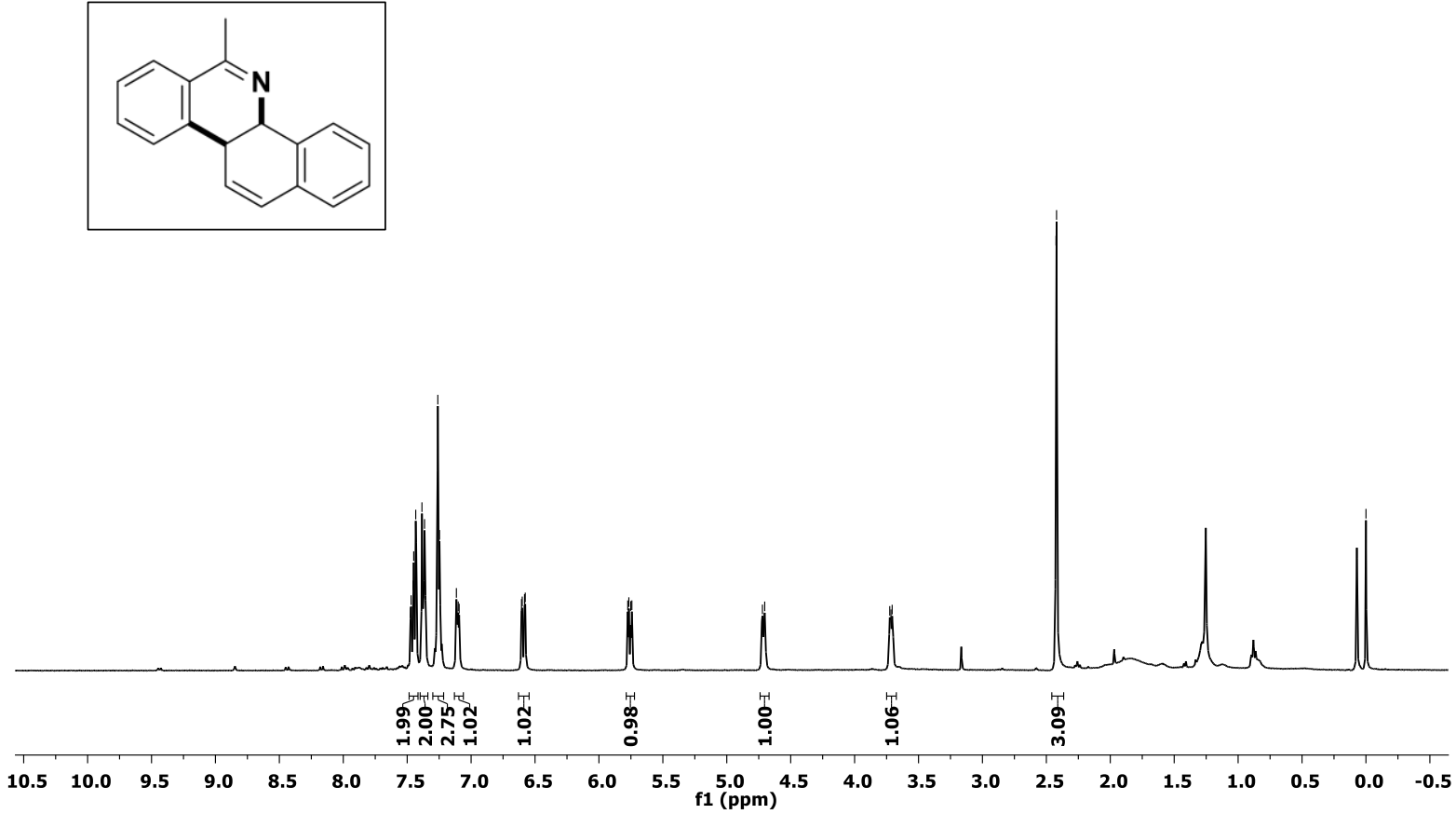

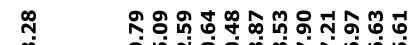

।

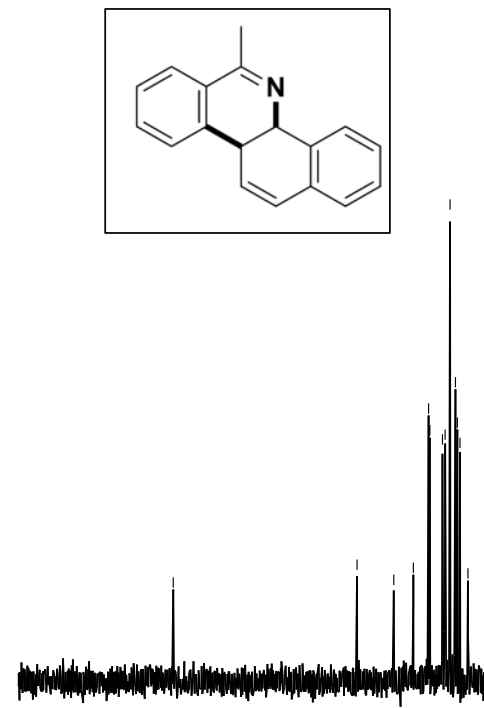

$\begin{array}{lllllllllll}180 & 170 & 160 & 150 & 140 & 130 & 120 & 110 & 100 & 90 & 80\end{array}$

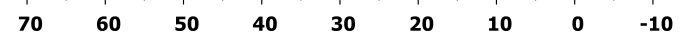


DEPT (135) NMR Spectrum of Compound 4a.

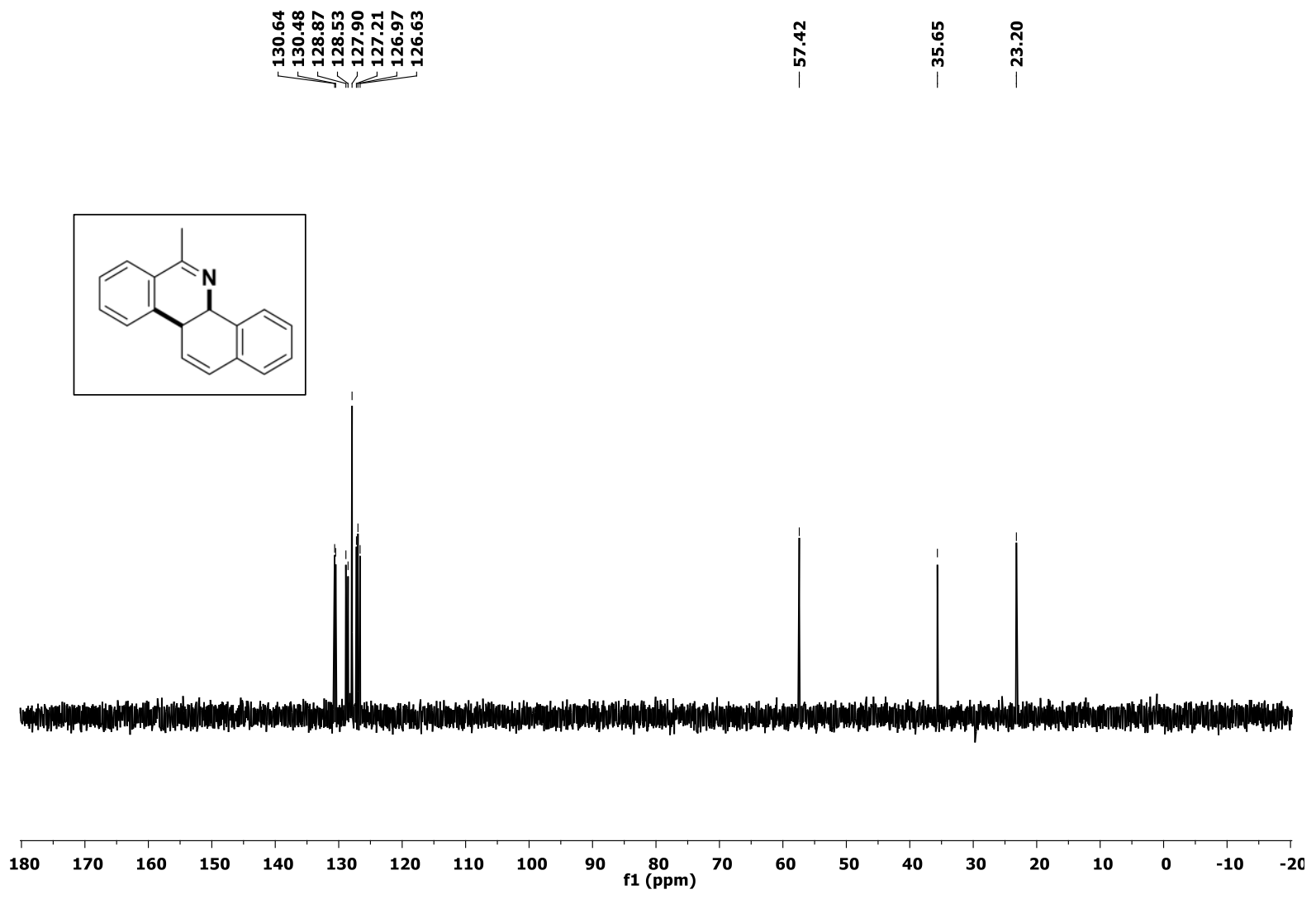


${ }^{1} \mathrm{H}$ and ${ }^{13} \mathrm{C}$ NMR Spectra of Compound $\mathbf{4 b}$.

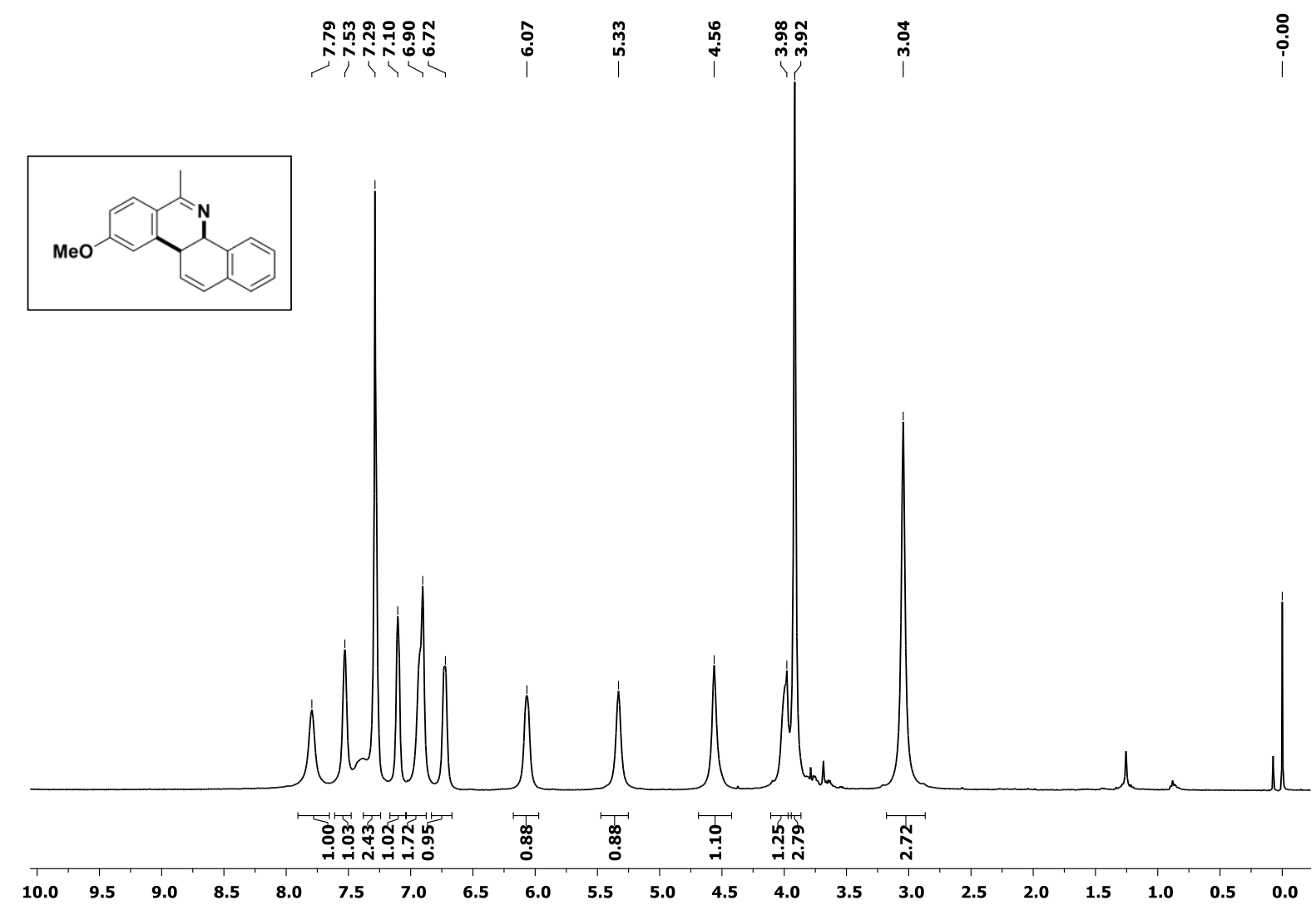

Fine shimming NMR expended Chemical shift 7.75 ppm to 3.0 ppm for better splitting

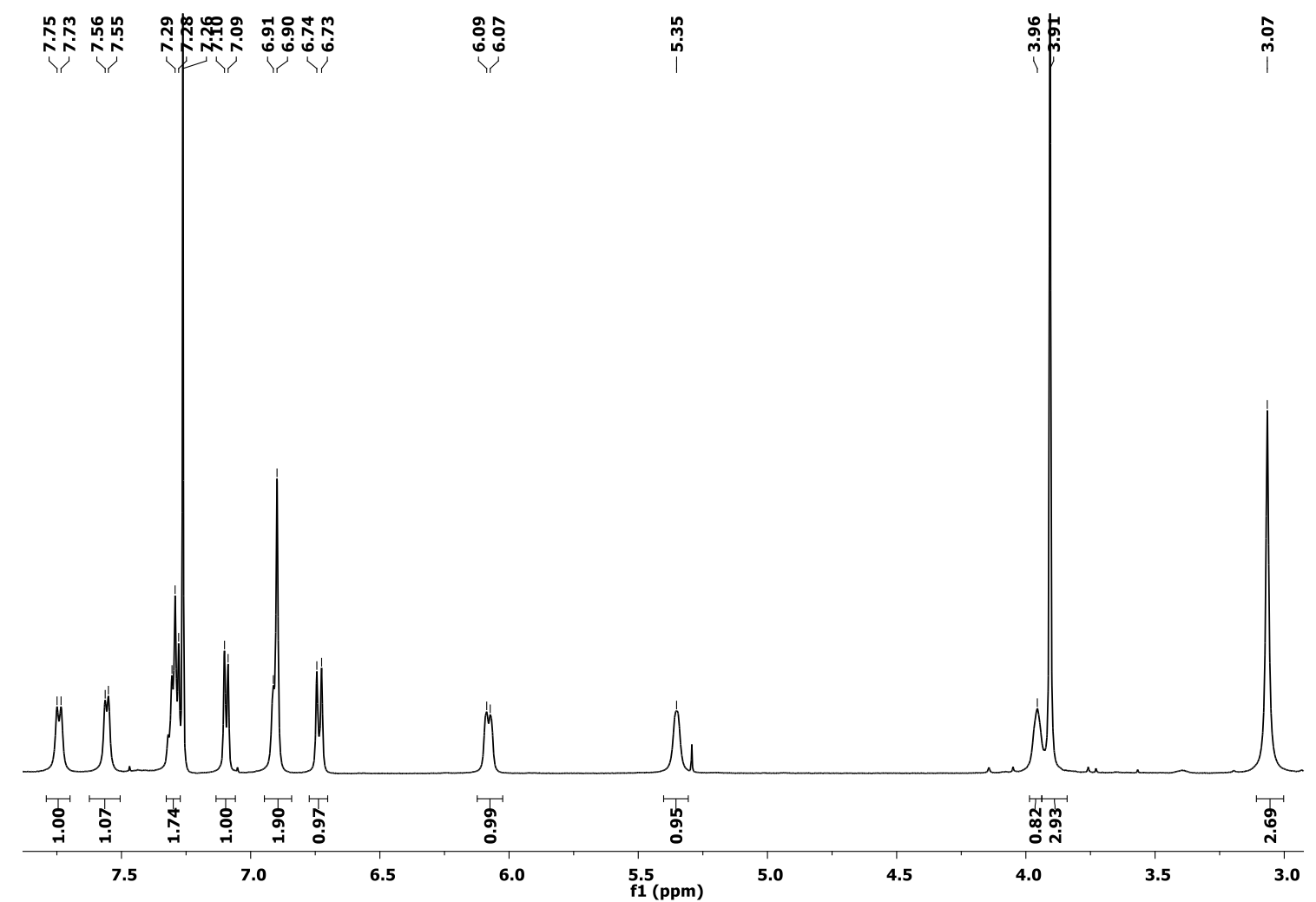



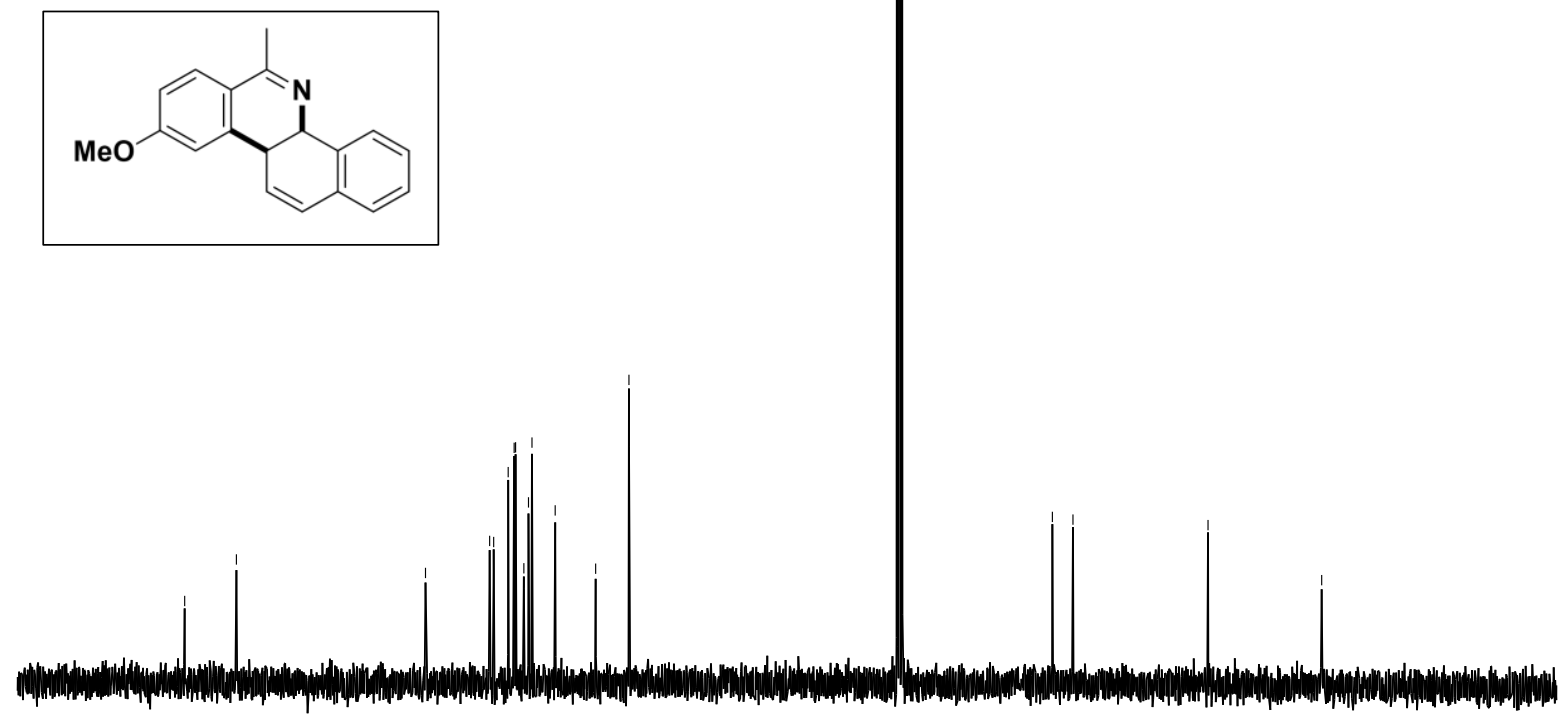

$\begin{array}{llllllllllllllllllllllll}190 & 180 & 170 & 160 & 150 & 140 & 130 & 120 & 110 & 100 & 90 & 80 & 70 & 60 & 50 & 40 & 30 & 20 & 10 & 0 & -10\end{array}$

DEPT (135) NMR Spectrum of Compound $\mathbf{4 b .}$
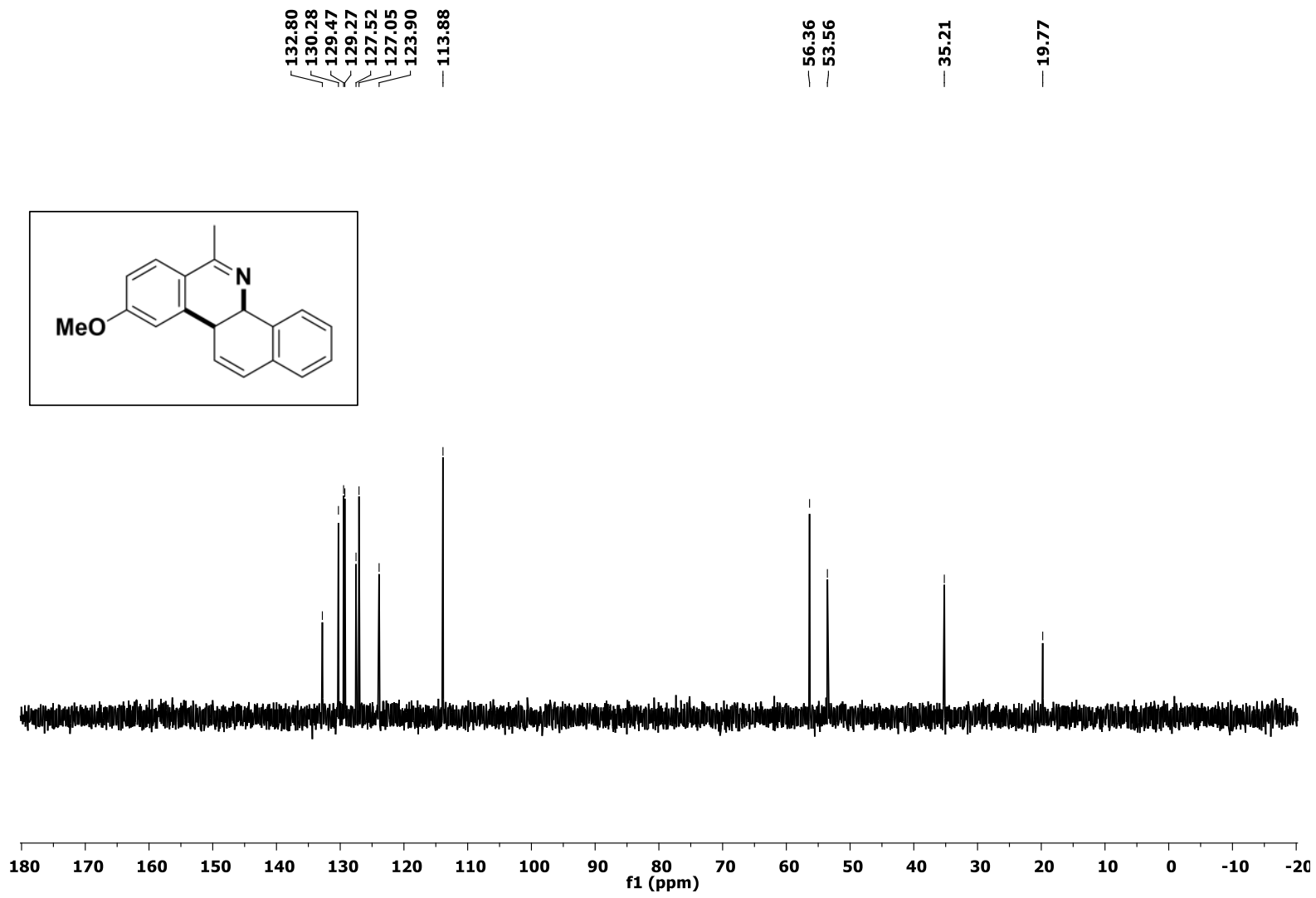
${ }^{1} \mathrm{H}$ and ${ }^{13} \mathrm{C}$ NMR Spectra of Compound $4 \mathbf{c}$.
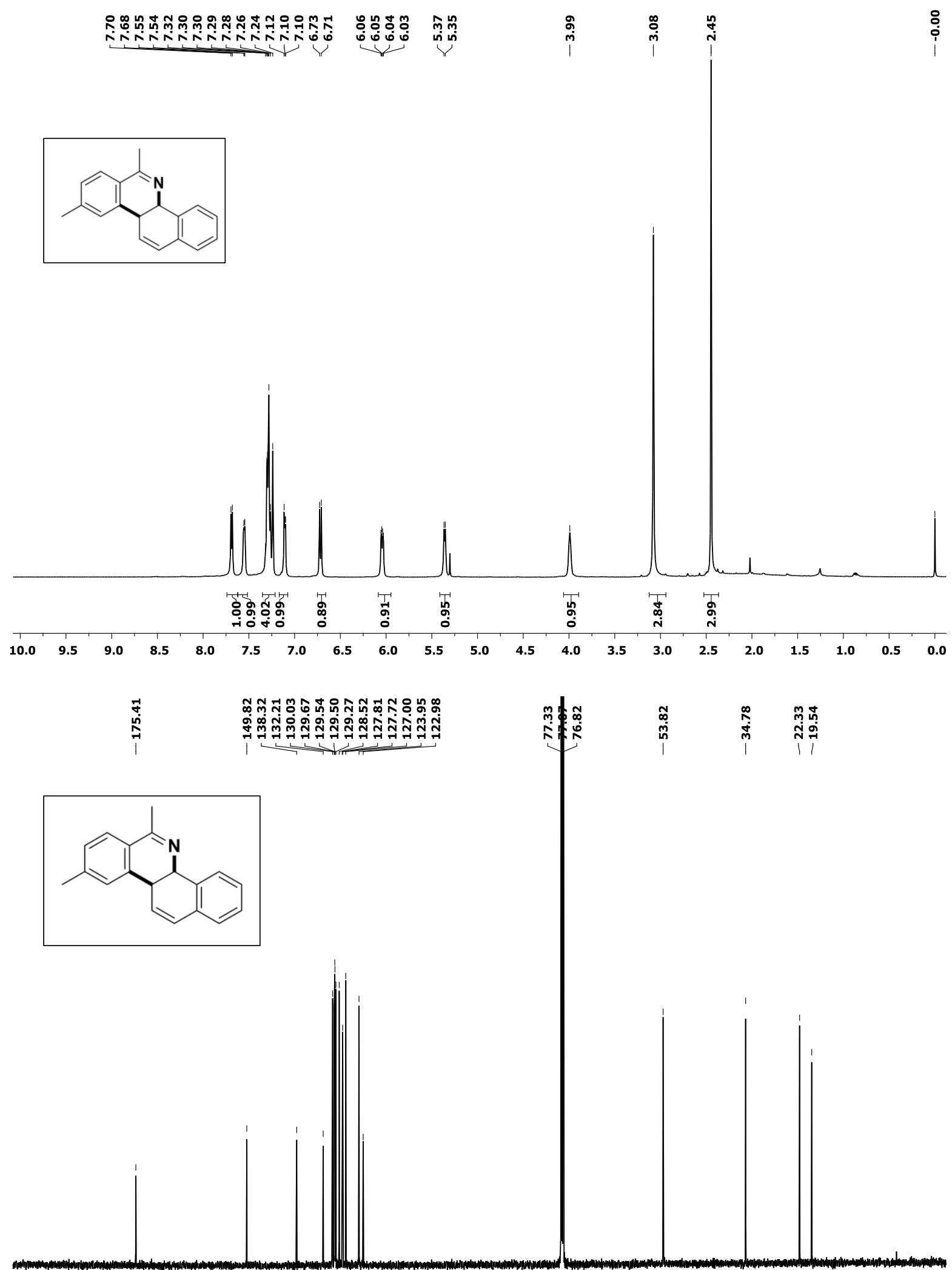

$\begin{array}{lllllllllllllllllllllll}200 & 190 & 180 & 170 & 160 & 150 & 140 & 130 & 120 & 110 & 100 & 90 & 80 & 70 & 60 & 50 & 40 & 30 & 20 & 10 & 0 & -10\end{array}$ 
DEPT (135) NMR Spectrum of Compound 4c.

ติดี

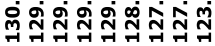

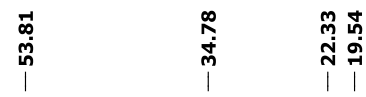
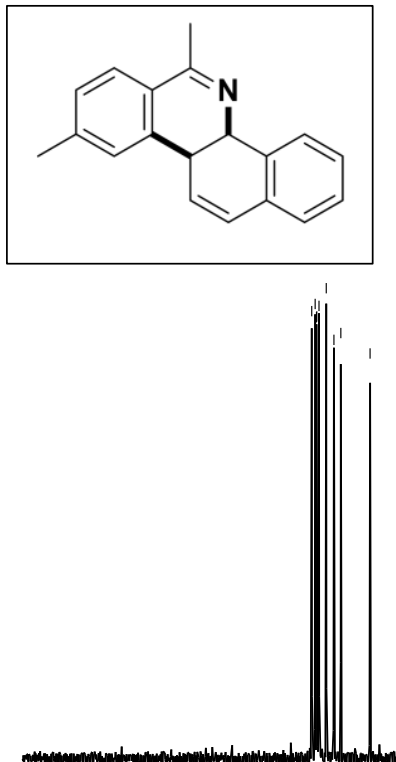

$\begin{array}{lllll}160 & 150 & 140 & 130 & 120\end{array}$

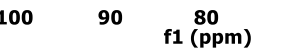

60

50

40

30

20

100 
${ }^{1} \mathrm{H}$ and ${ }^{13} \mathrm{C}$ NMR Spectra of Compound $\mathbf{4 d}$.

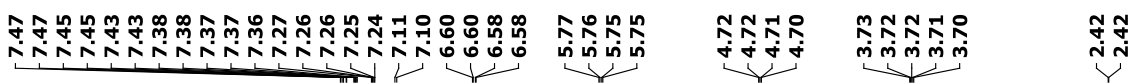
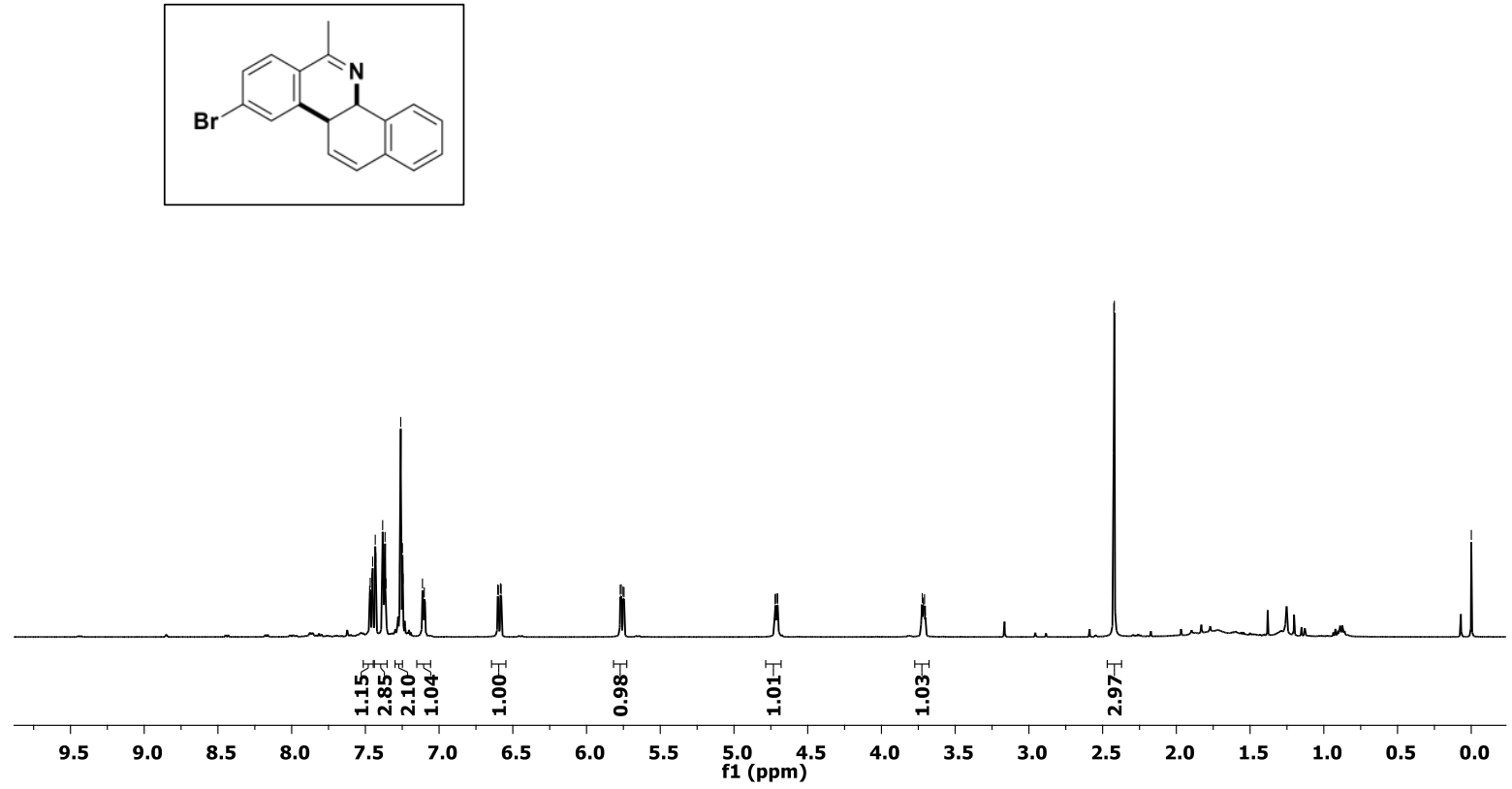

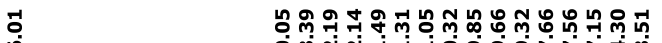

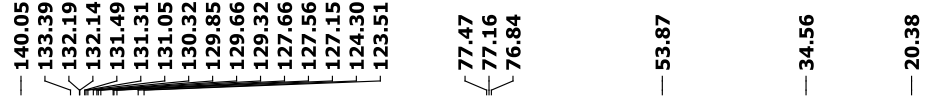

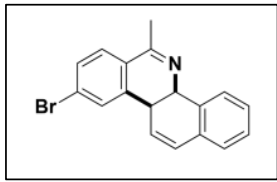

$\begin{array}{lllllllllll}190 & 180 & 170 & 160 & 150 & 140 & 130 & 120 & 110 & 100 & \begin{array}{c}90 \\ \text { f1 }\end{array}(\mathrm{ppm})\end{array}$ 
DEPT (135) NMR Spectrum of Compound 4d.

马ㅇำㅁำ

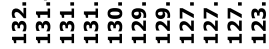

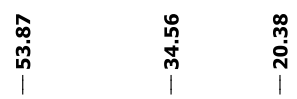
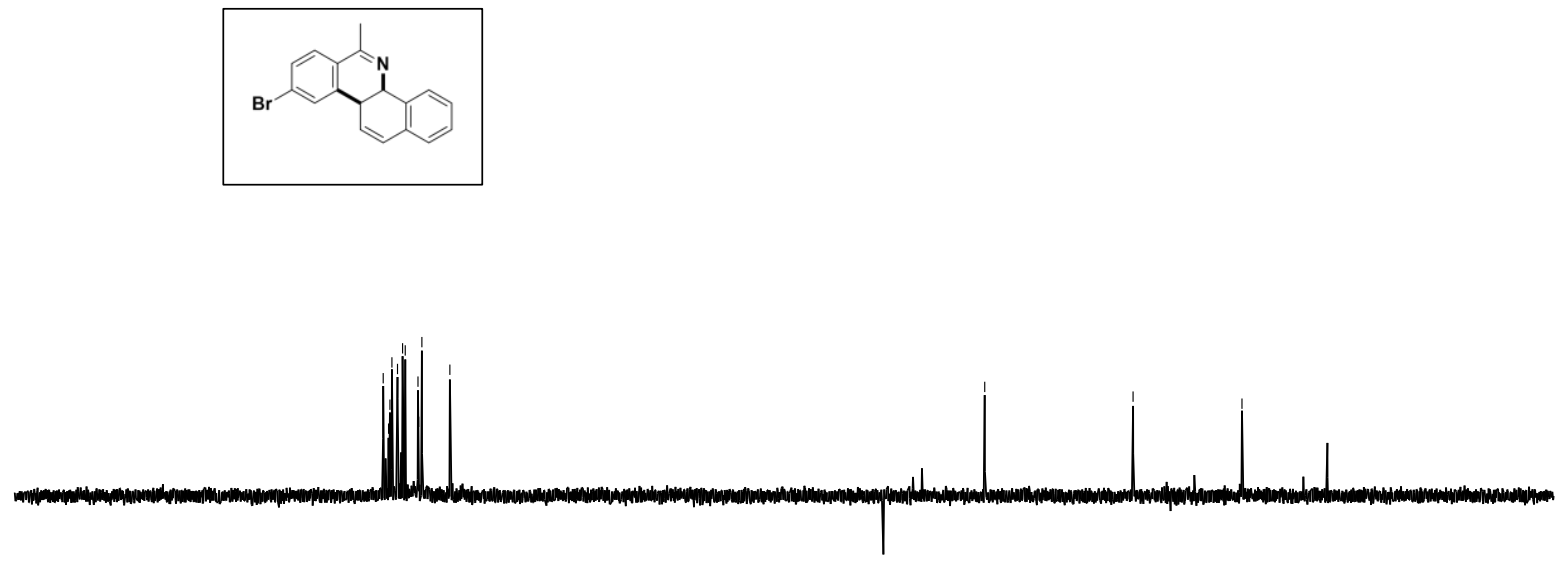

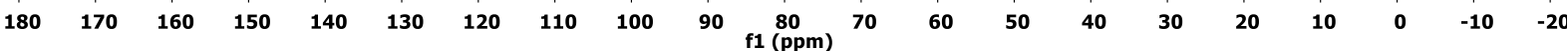


${ }^{1} \mathrm{H}$ and ${ }^{13} \mathrm{C}$ NMR Spectra of Compound 4e.
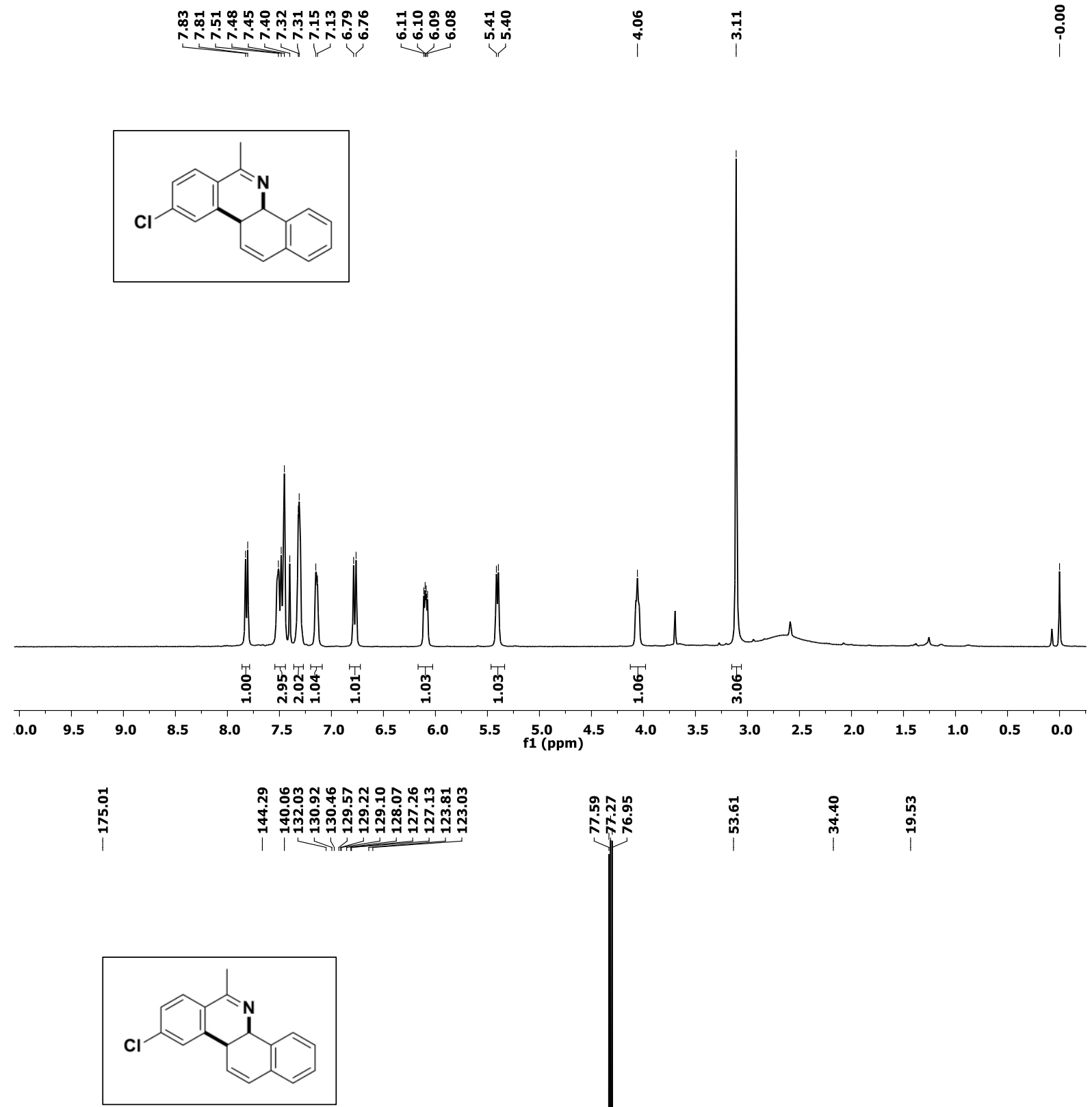

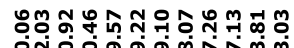

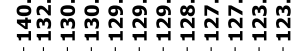

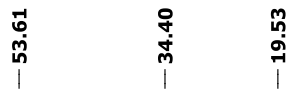

$\begin{array}{lllllllllll}180 & 170 & 160 & 150 & 140 & 130 & 120 & 110 & 100 & \begin{array}{l}90 \\ f 1(\mathrm{ppm})\end{array} & 80\end{array}$

$\begin{array}{lllllllll}70 & 60 & 50 & 40 & 30 & 20 & 10 & 0 & -10\end{array}$ 
${ }^{1} \mathrm{H}$ and ${ }^{13} \mathrm{C}$ NMR Spectra of Compound $4 \mathbf{f}$.

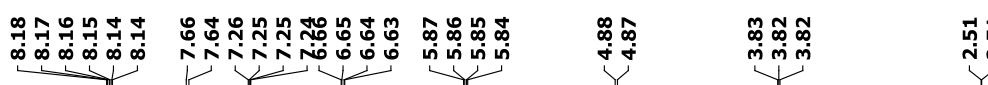

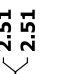
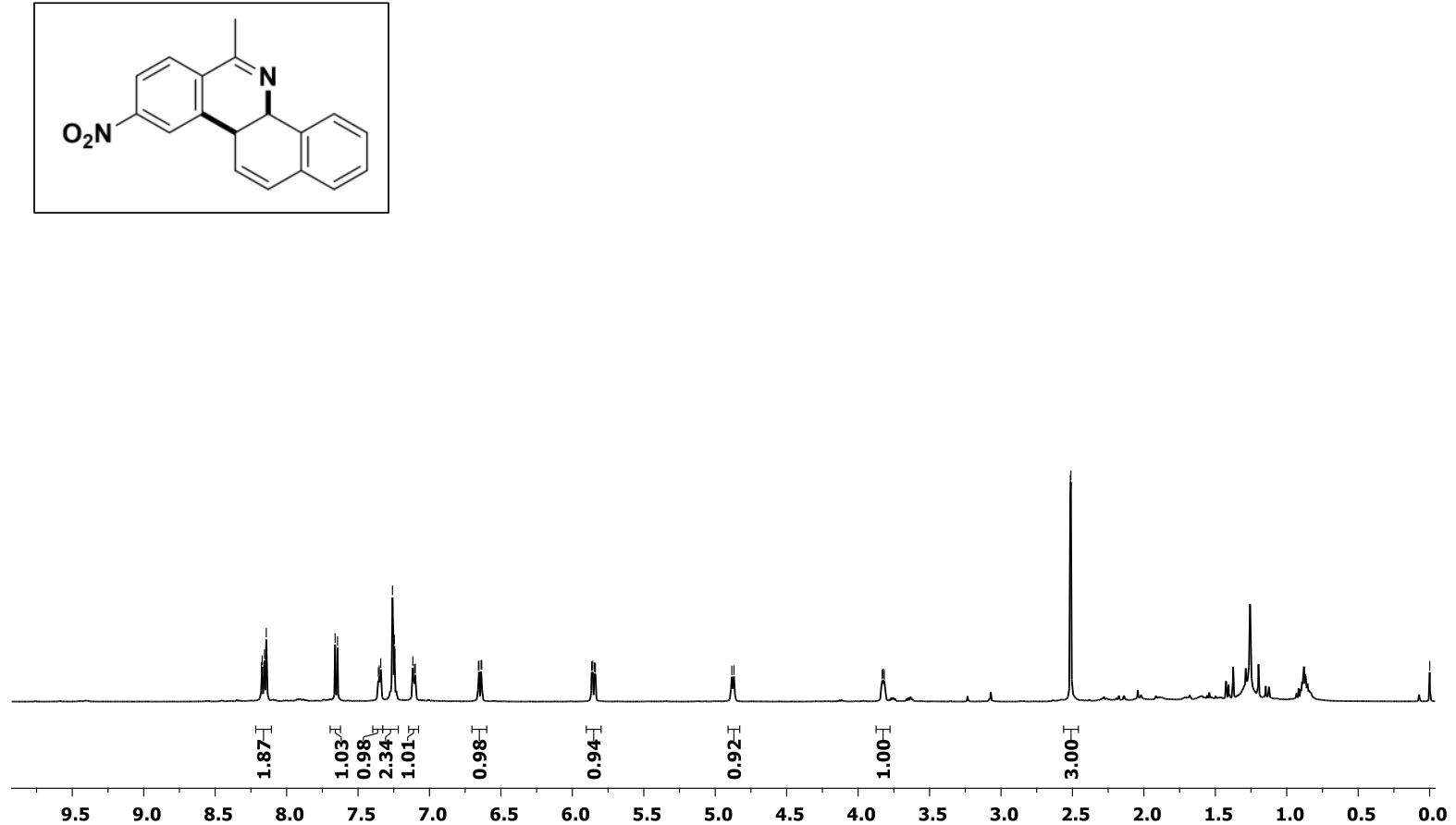

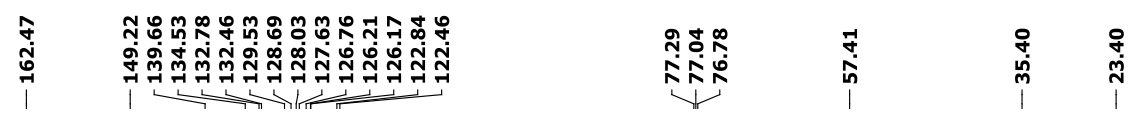
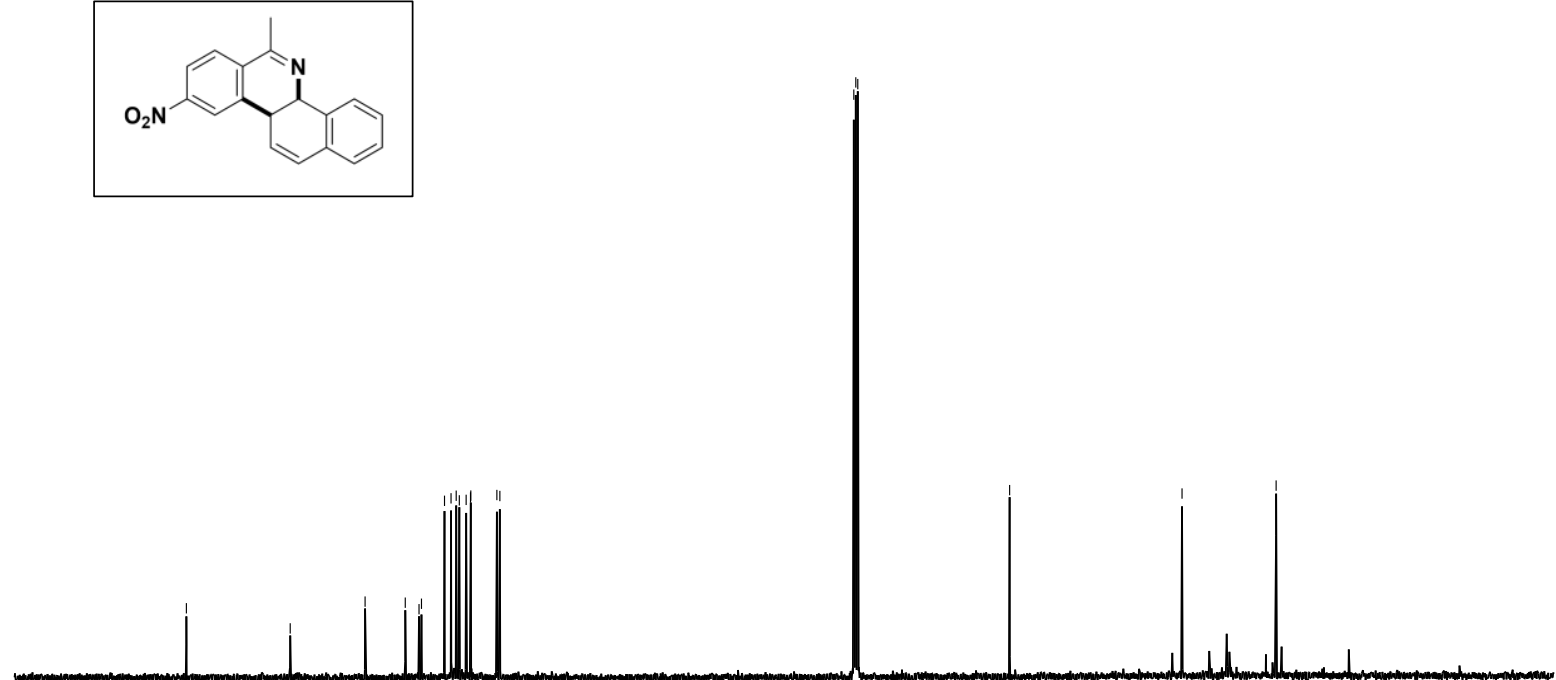

$\begin{array}{lllllllllllllllllllll}180 & 170 & 160 & 150 & 140 & 130 & 120 & 110 & 100 & 90 & 80 & 70 & 60 & 50 & 40 & 30 & 20 & 10 & 0 & -10\end{array}$ 
DEPT (135) NMR Spectrum of Compound $4 \mathbf{4 f}$.

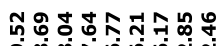

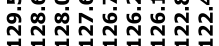

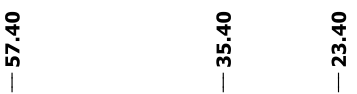
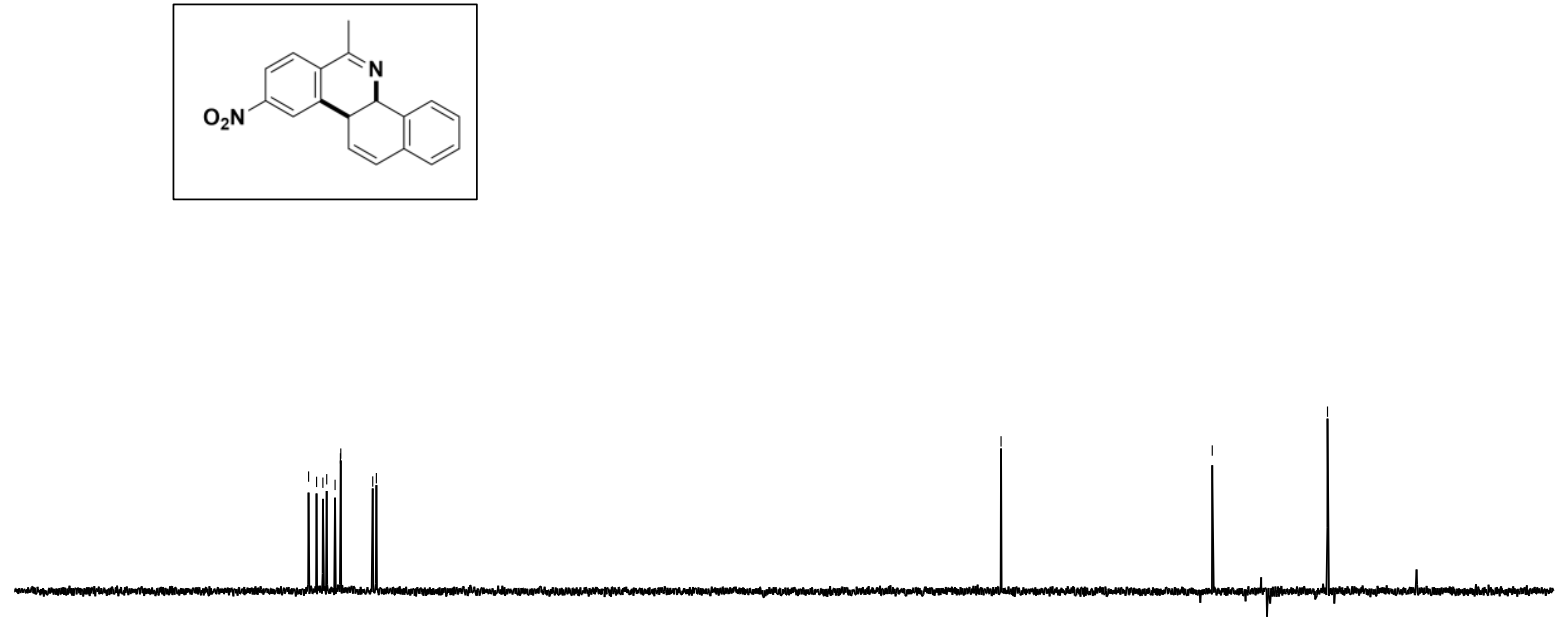

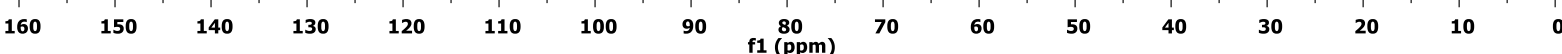


${ }^{1} \mathrm{H}$ and ${ }^{13} \mathrm{C}$ NMR Spectra of Compound $\mathbf{4 g}$.

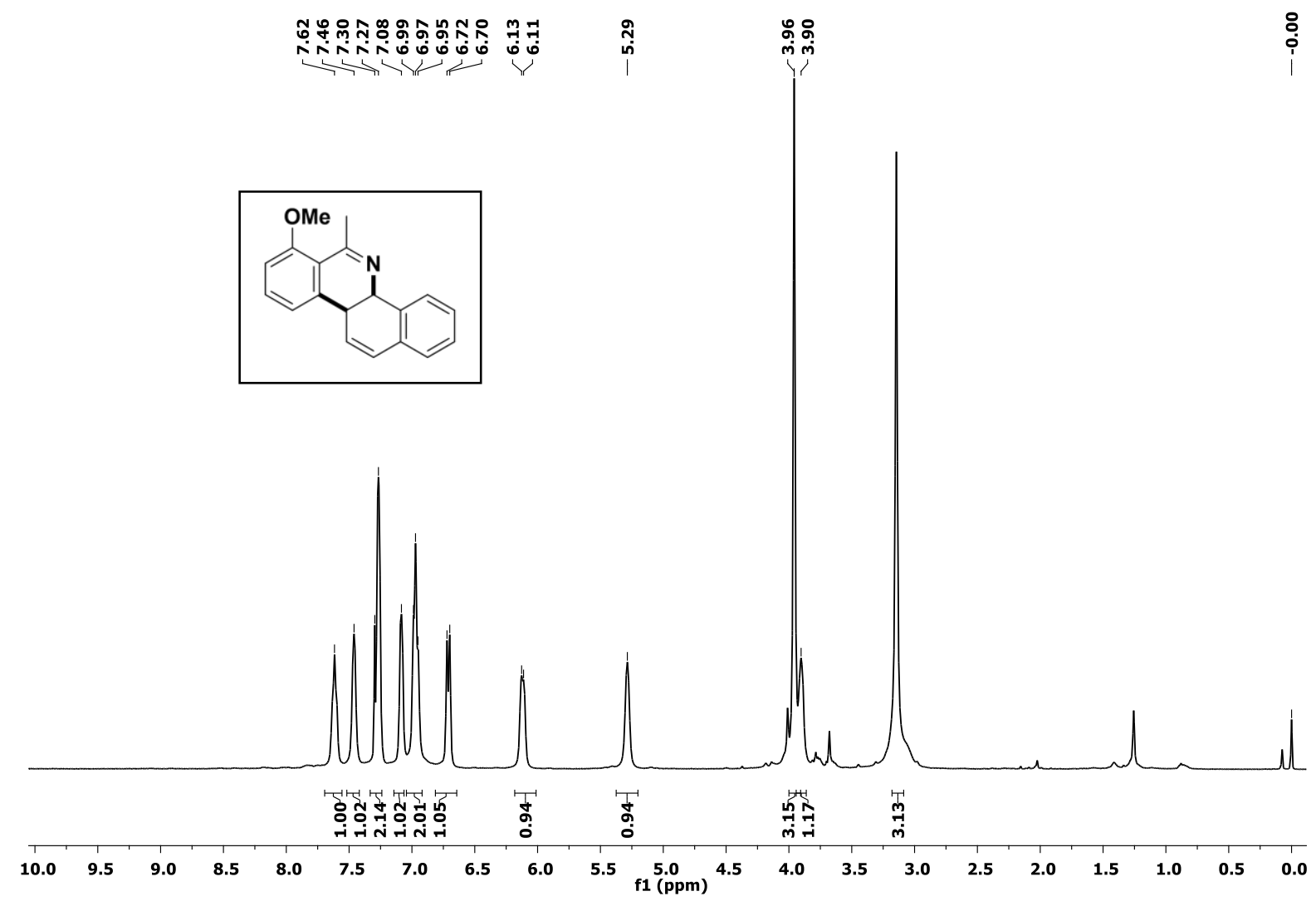

Fine shimming NMR expended Chemical shift 7.6 ppm to $3.0 \mathrm{ppm}$ for better splitting

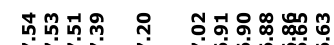

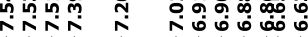

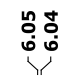

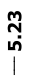

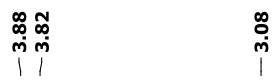

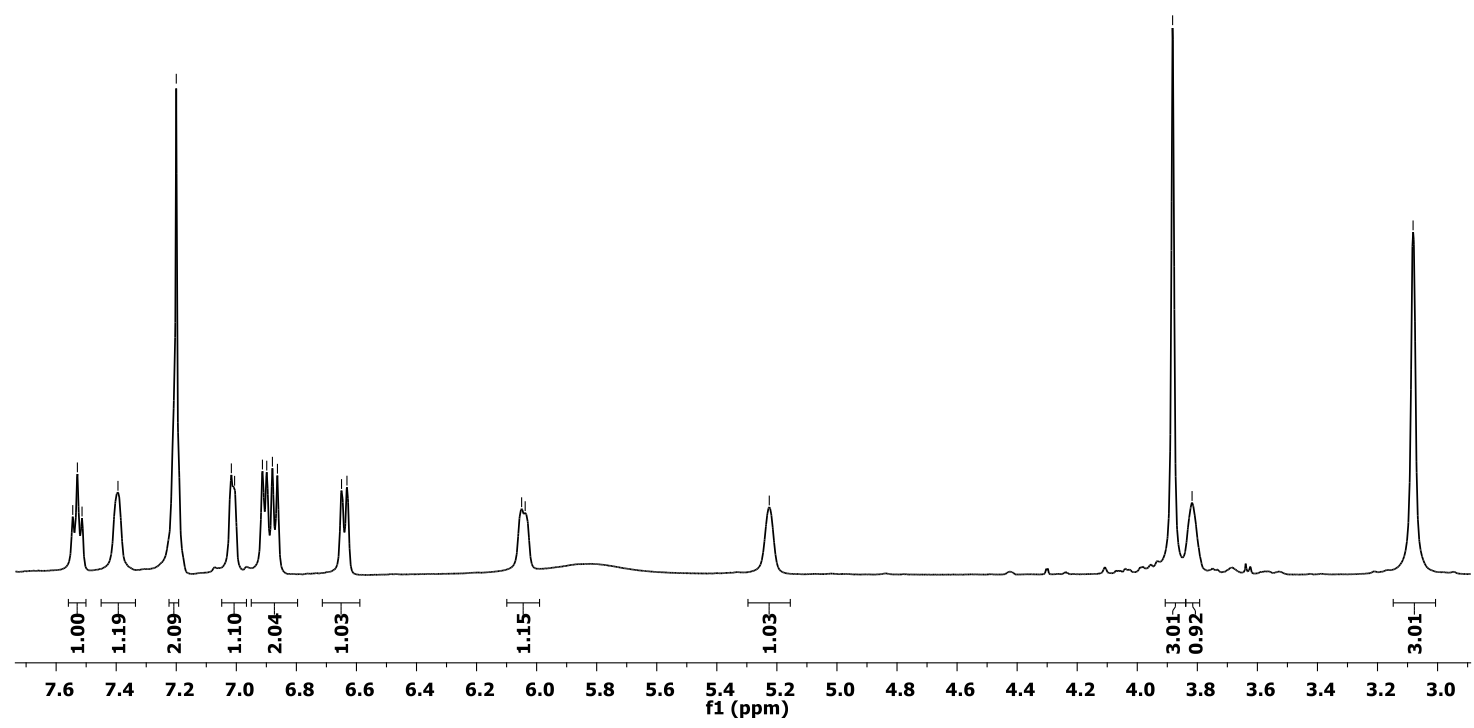




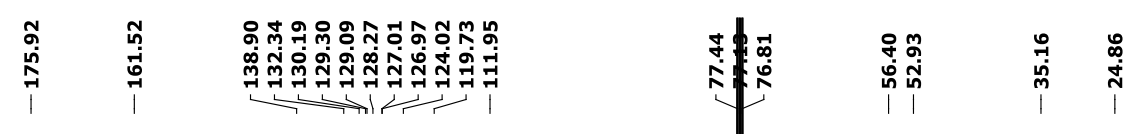
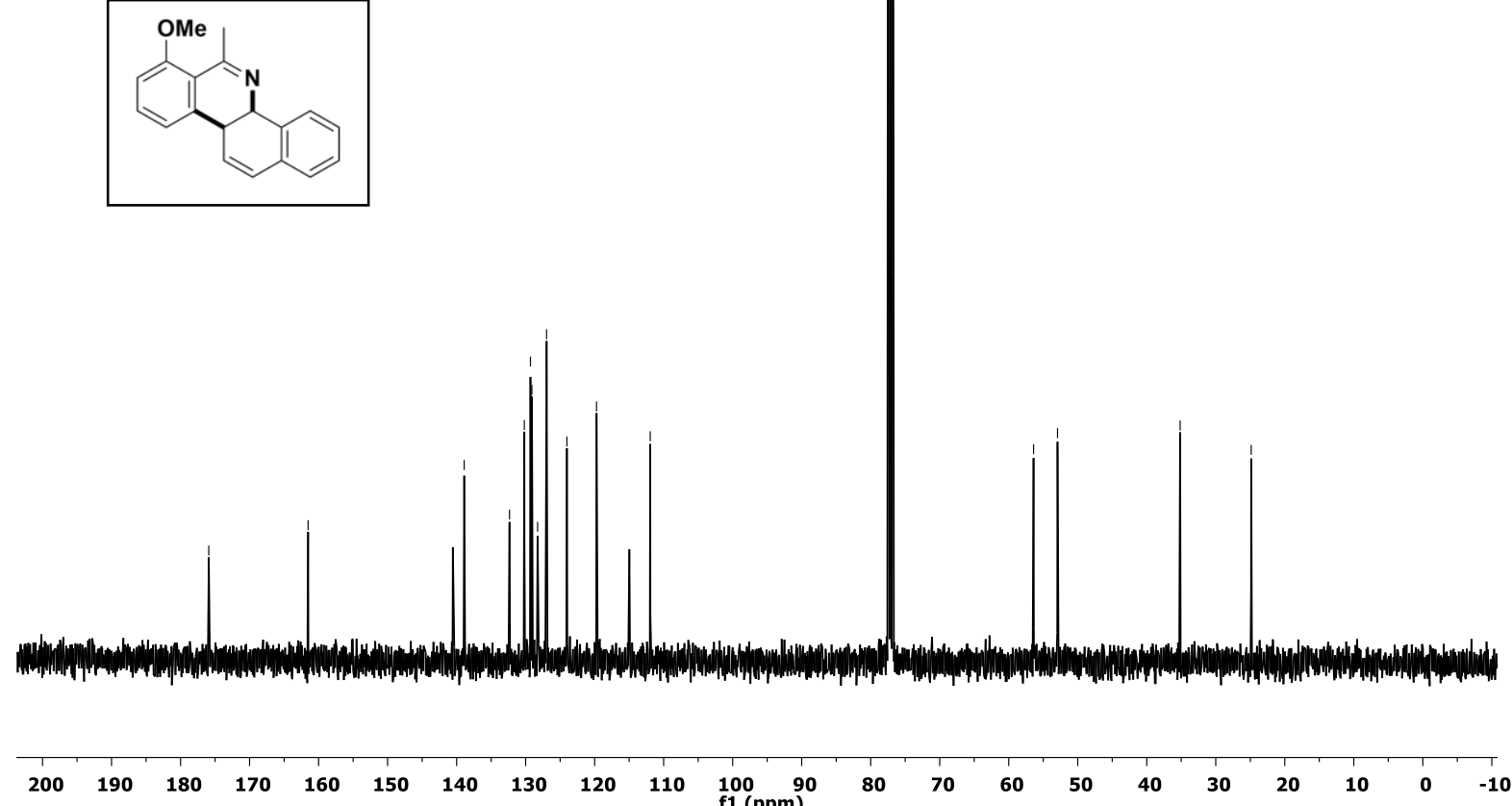

DEPT (135) NMR Spectrum of Compound 4g.

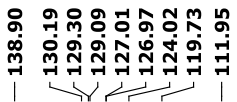

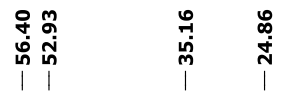
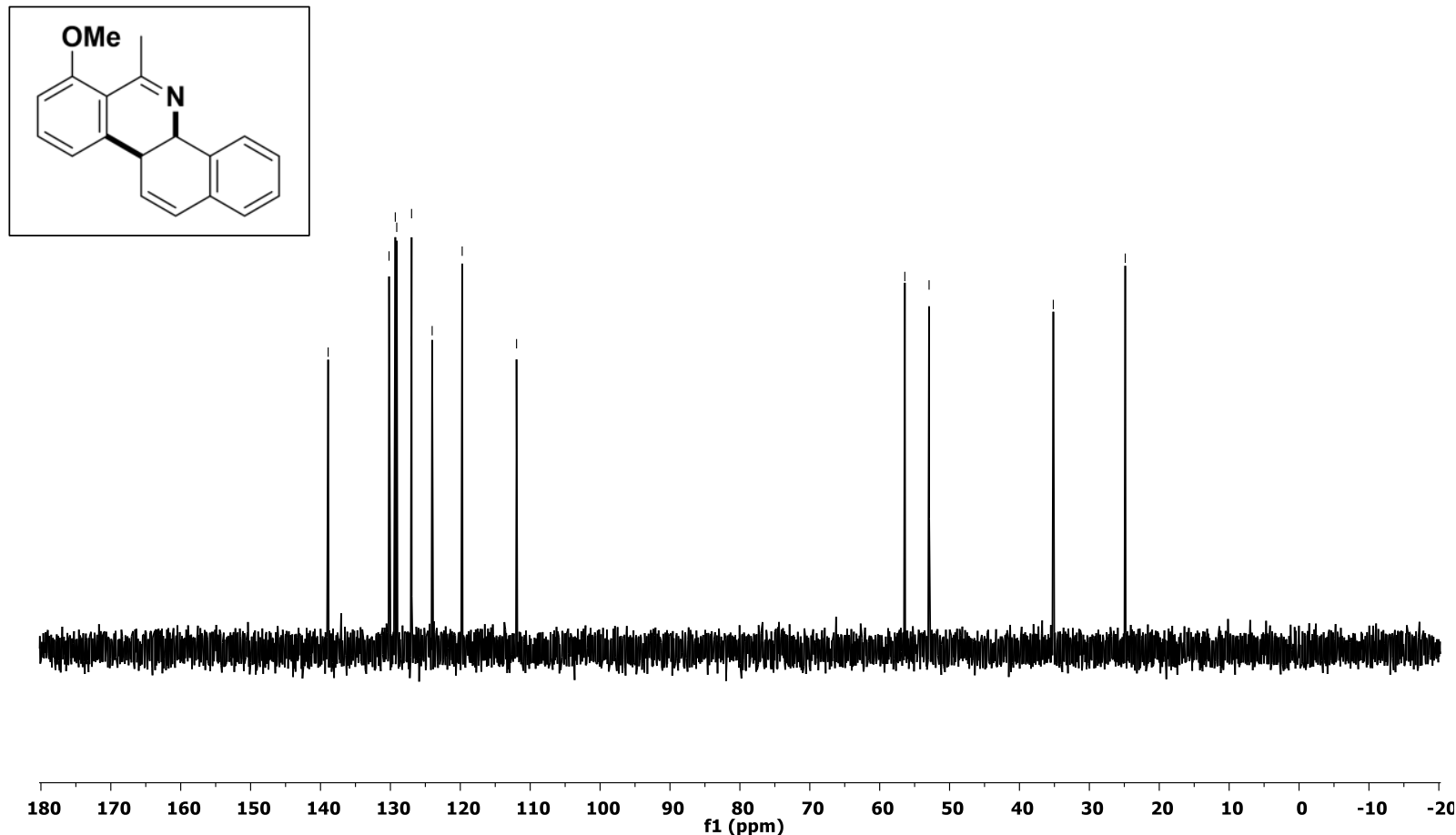
${ }^{1} \mathrm{H}$ and ${ }^{13} \mathrm{C}$ NMR Spectra of Compound $\mathbf{4 h}$.

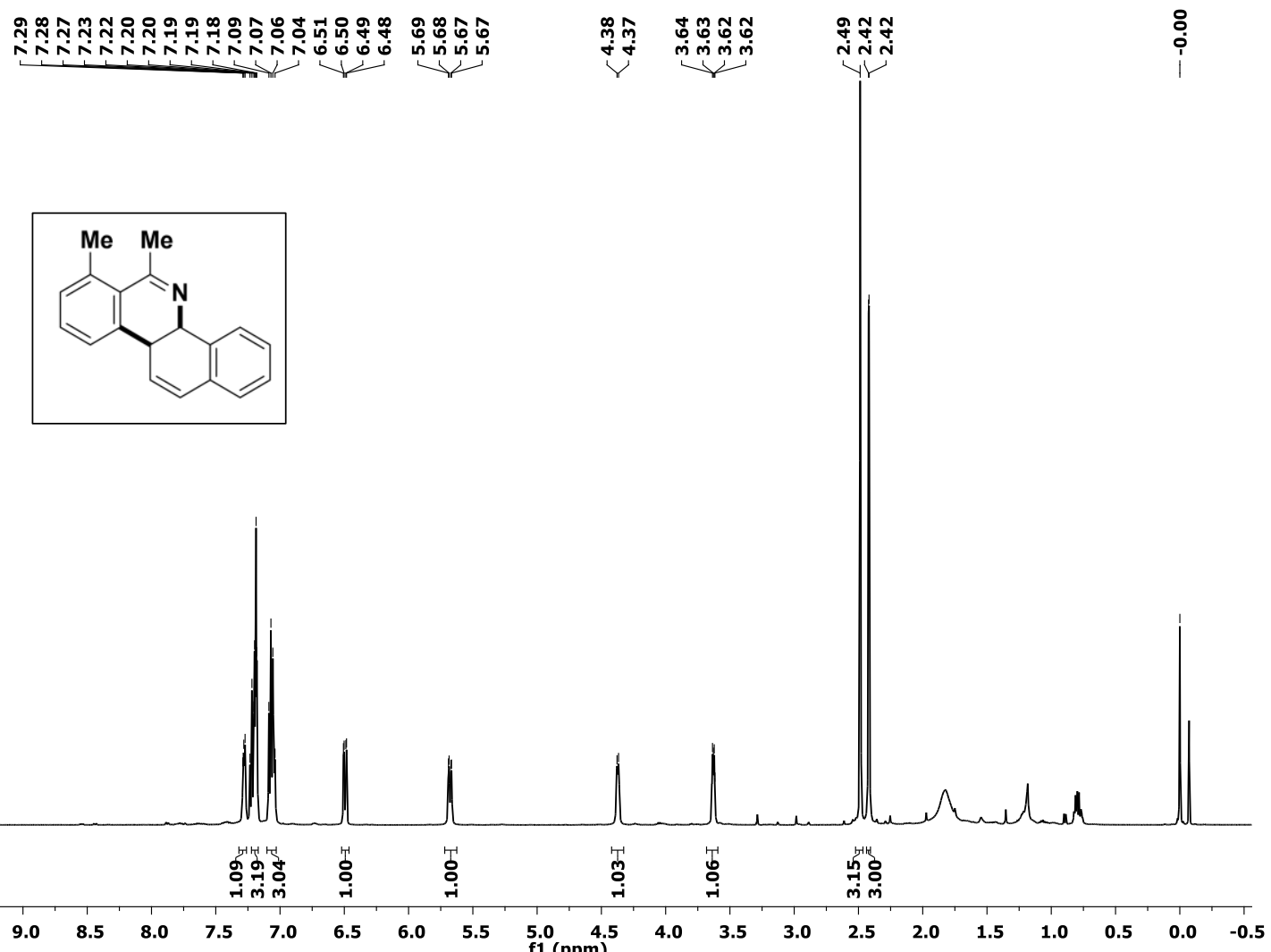

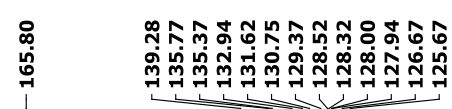

策尔

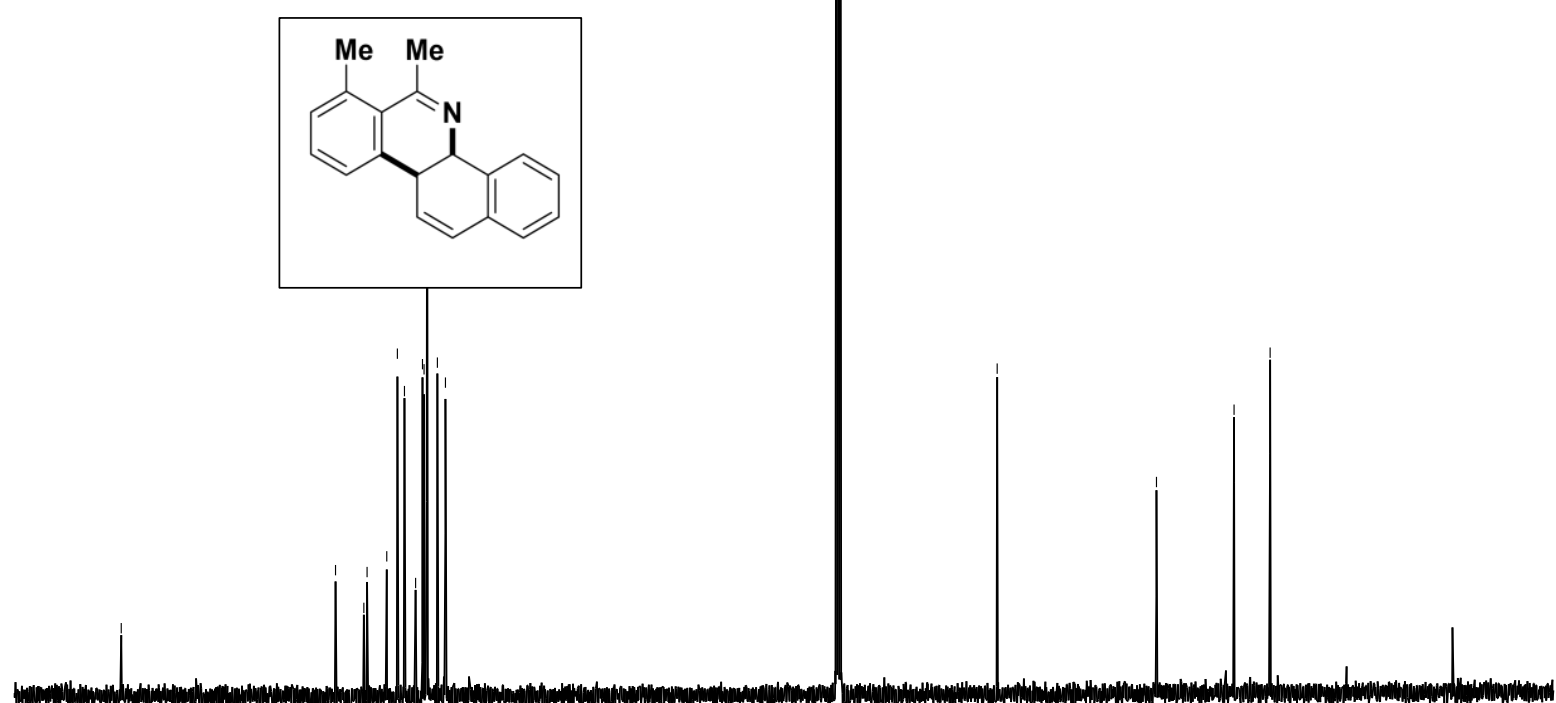

$\begin{array}{llllllllll}170 & 160 & 150 & 140 & 130 & 120 & 110 & 100 & 90 & 80\end{array}$ 
DEPT (135) NMR Spectrum of Compound $\mathbf{4 h}$.

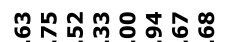

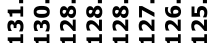

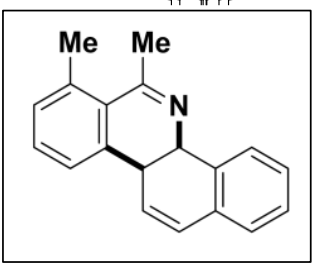

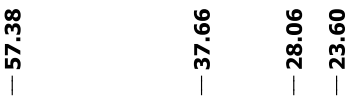

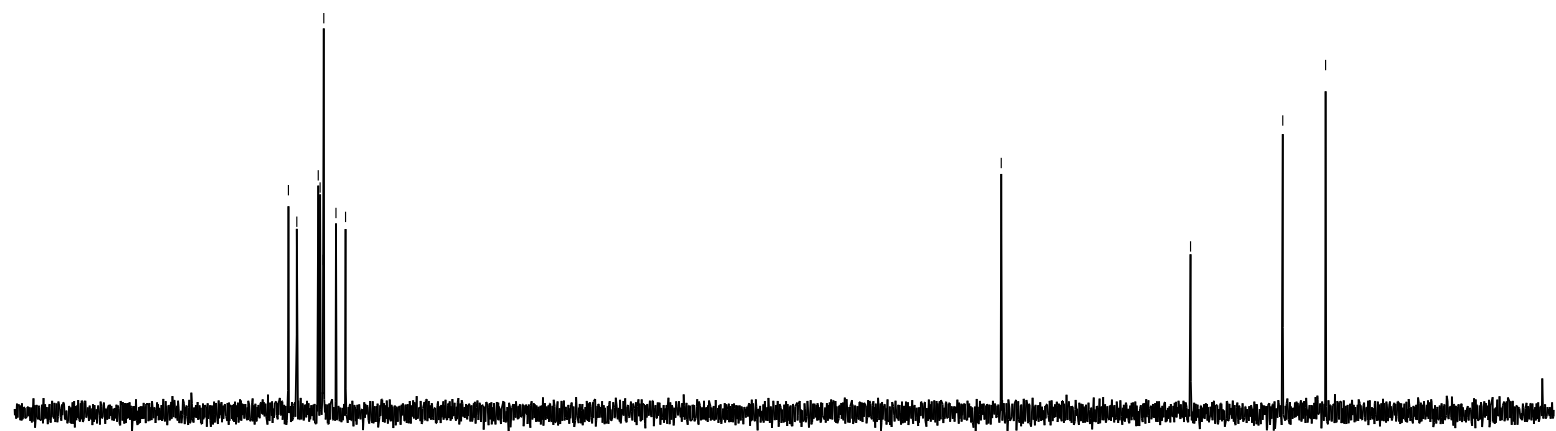

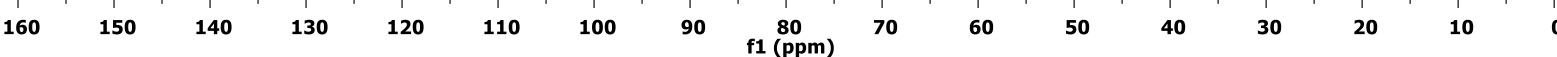


${ }^{1} \mathrm{H}$ and ${ }^{13} \mathrm{C}$ NMR Spectra of Compound $\mathbf{4 i}$.

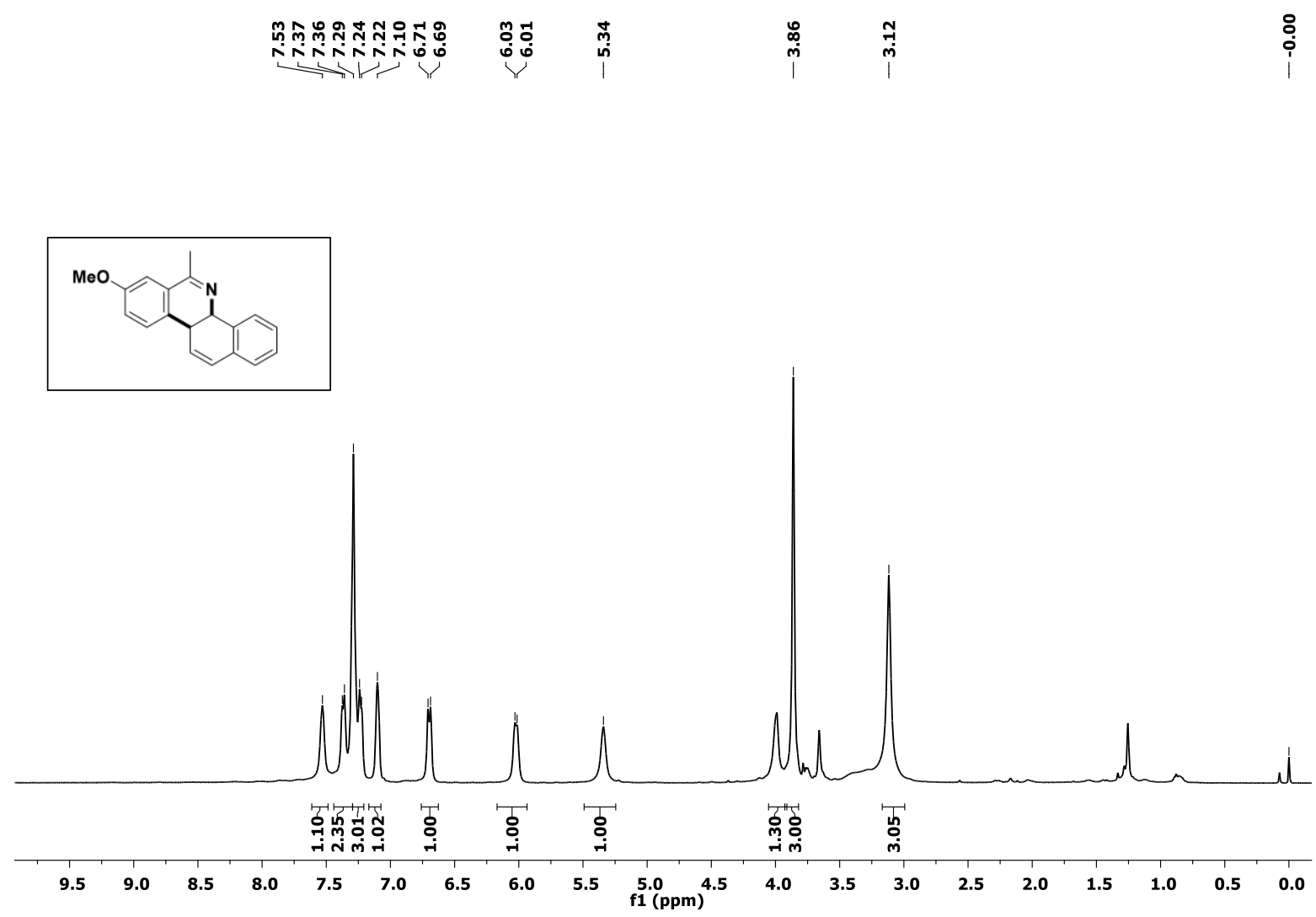

Fine shimming NMR expended: Chemical shift 7.54 ppm to 3.0 ppm for better splitting

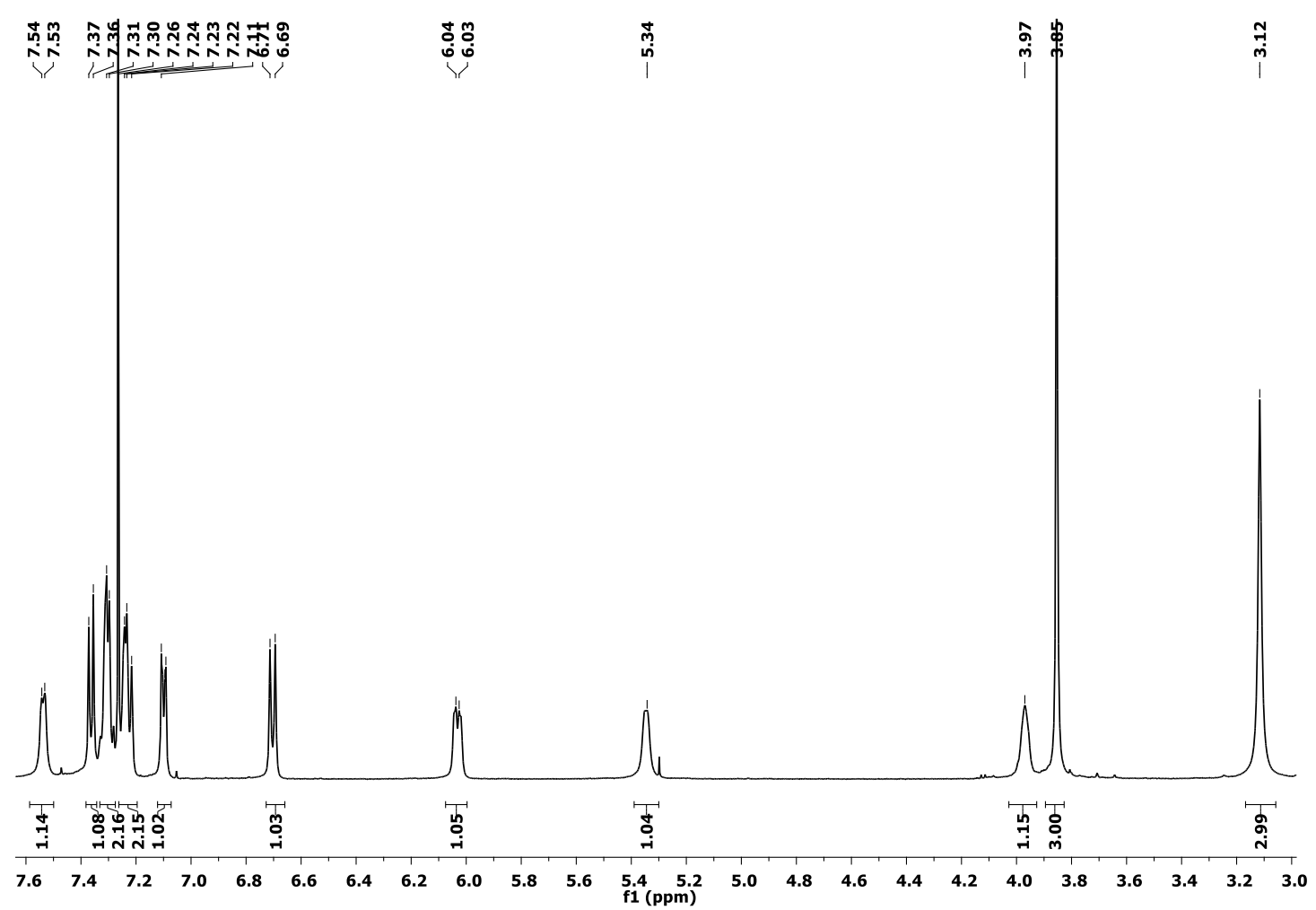




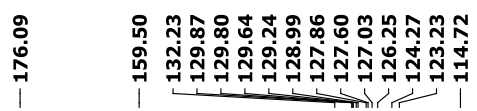
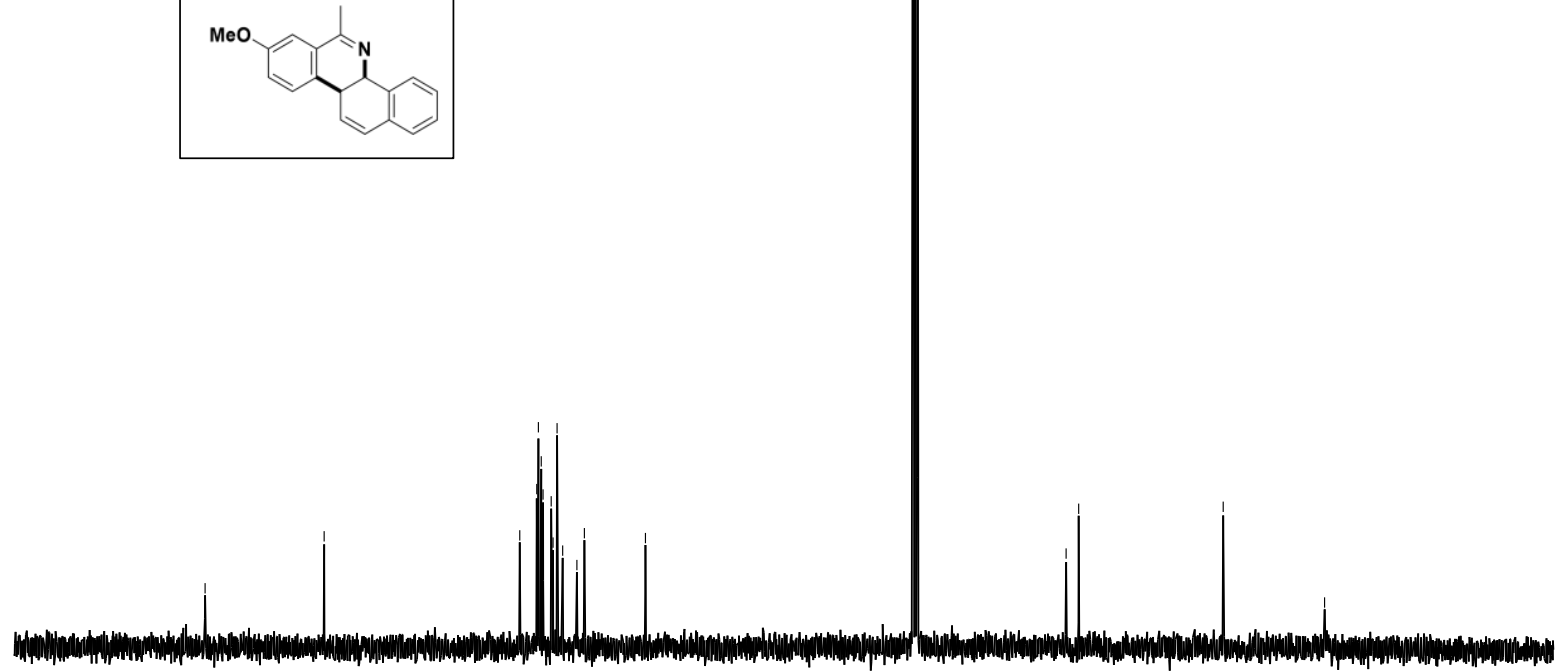

$\begin{array}{lllllllllll}200 & 190 & 180 & 170 & 160 & 150 & 140 & 130 & 120 & 110 & \begin{array}{l}100 \\ \mathrm{f} 1(\mathrm{ppm})\end{array}\end{array}$

DEPT (135) NMR Spectrum of Compound 4i.

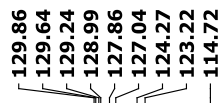

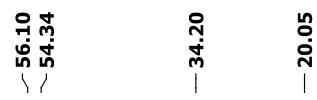
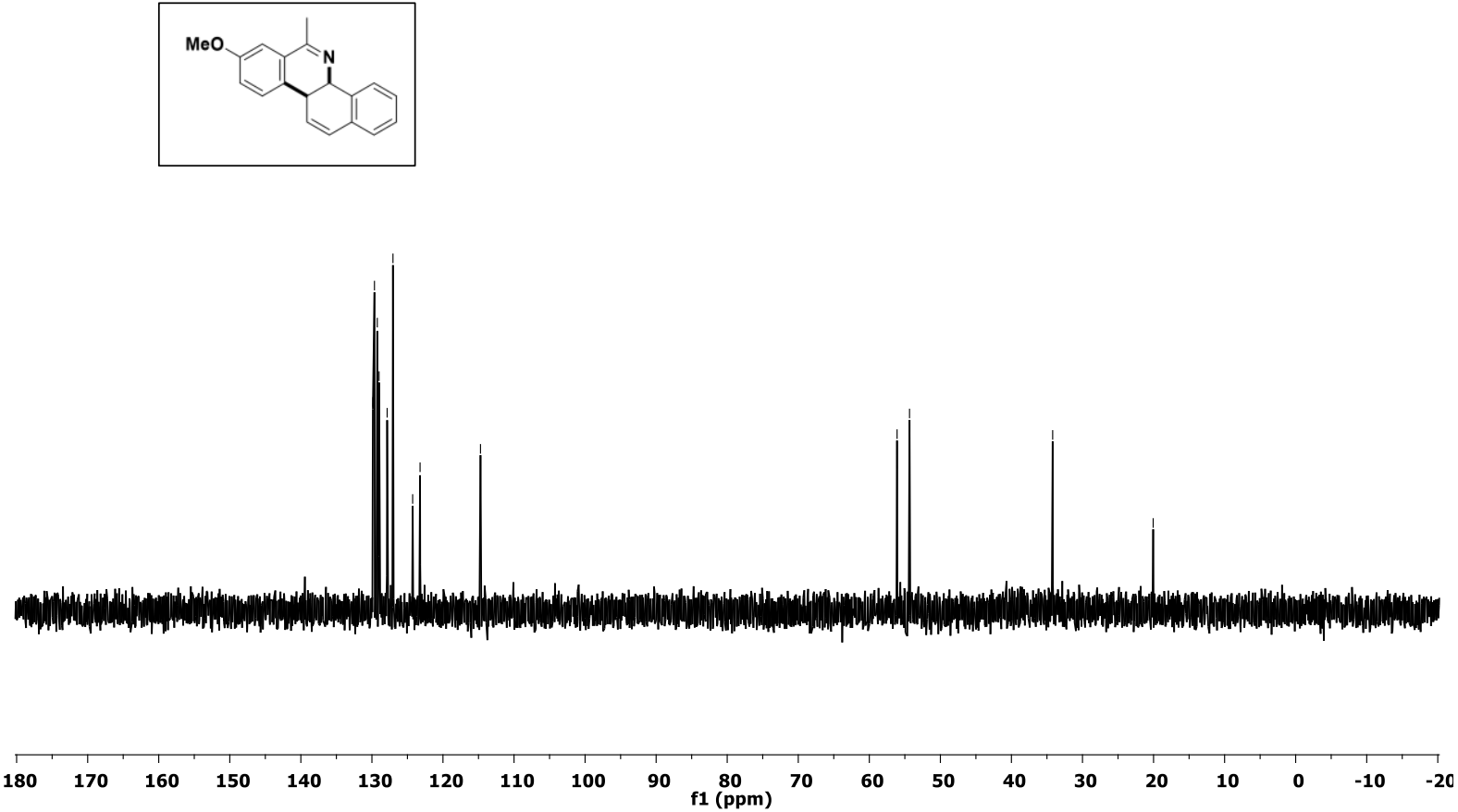
${ }^{1} \mathrm{H}$ and ${ }^{13} \mathrm{C}$ NMR Spectra of Compound $\mathbf{4} \mathbf{j}$.

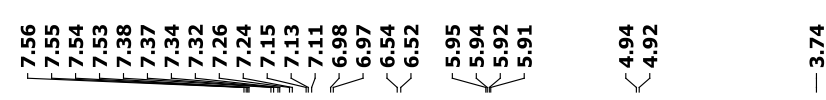
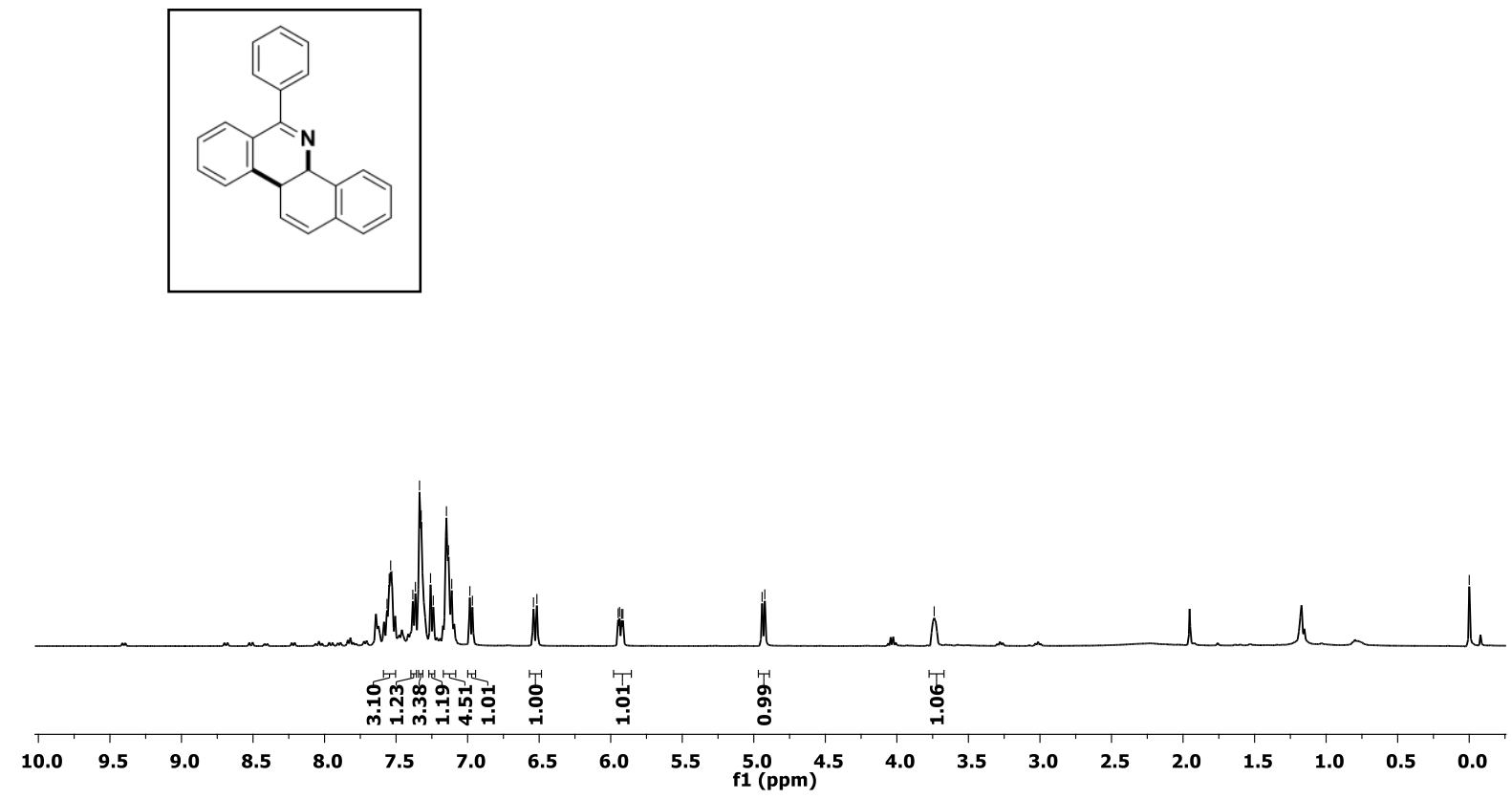

l

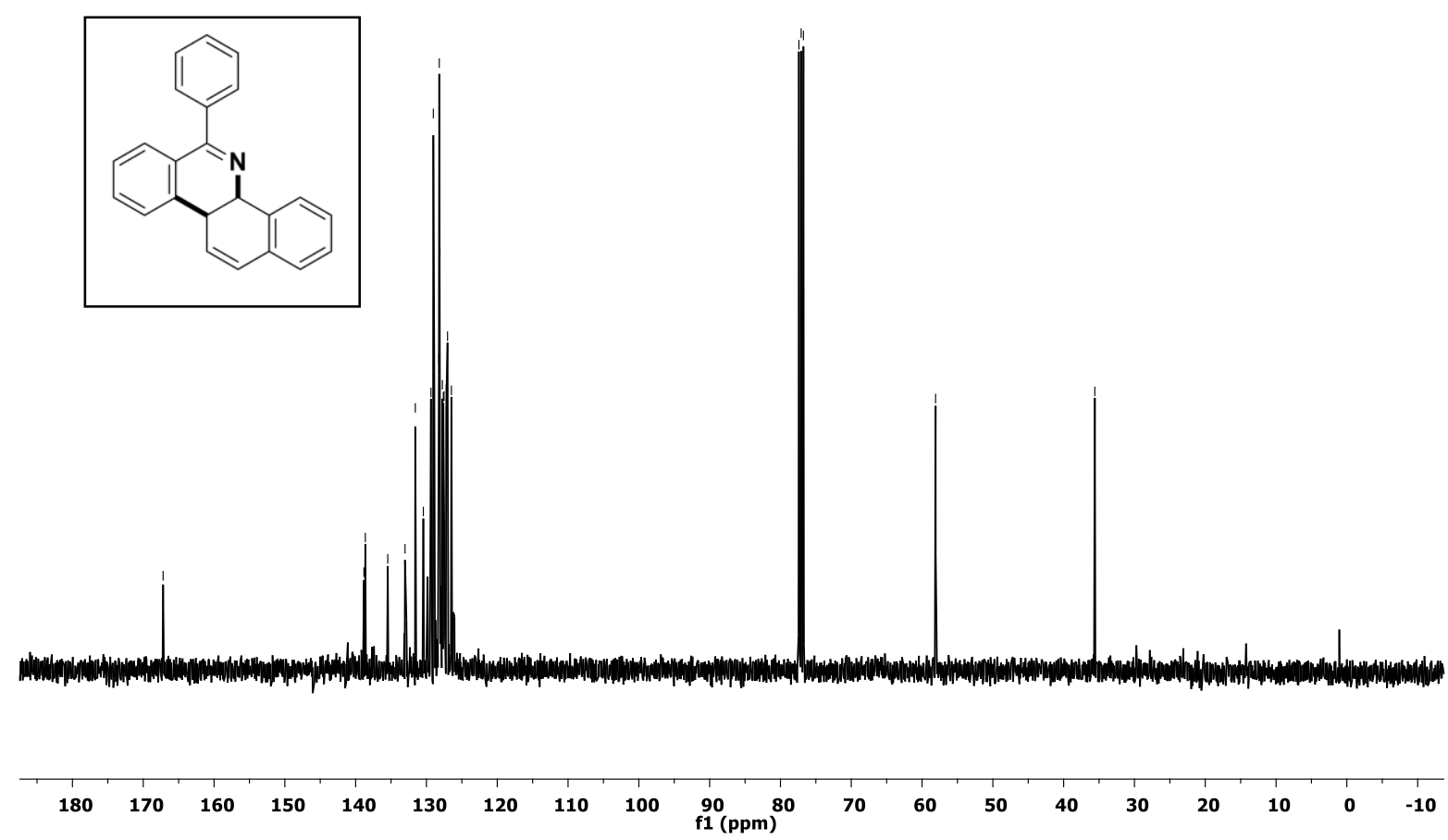


DEPT (135) NMR Spectrum of Compound $\mathbf{4 j}$.

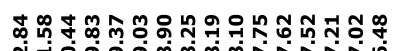

نं

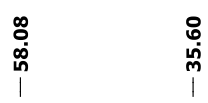

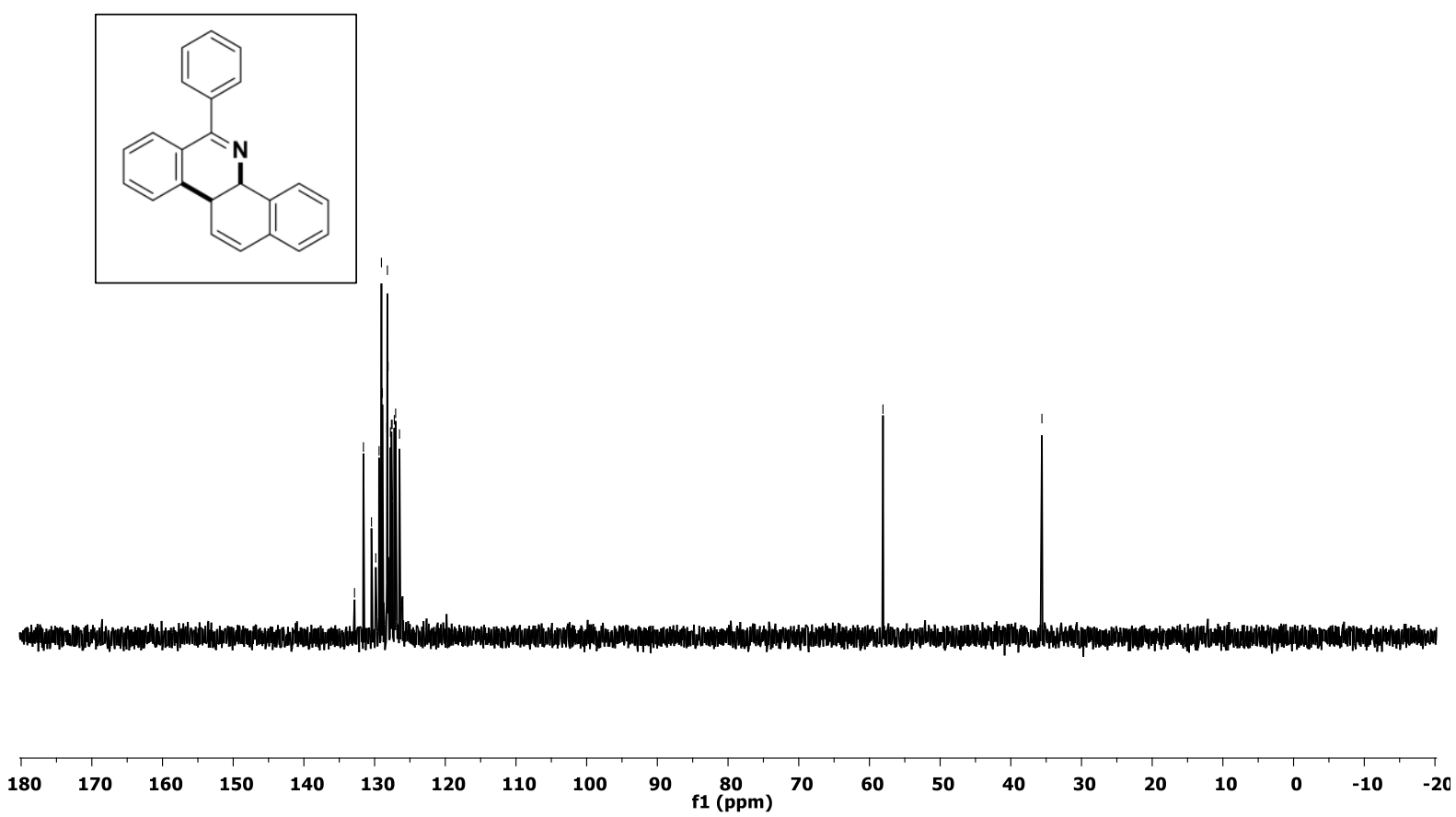


${ }^{1} \mathrm{H}$ and ${ }^{13} \mathrm{C}$ NMR Spectra of Compound 5a.

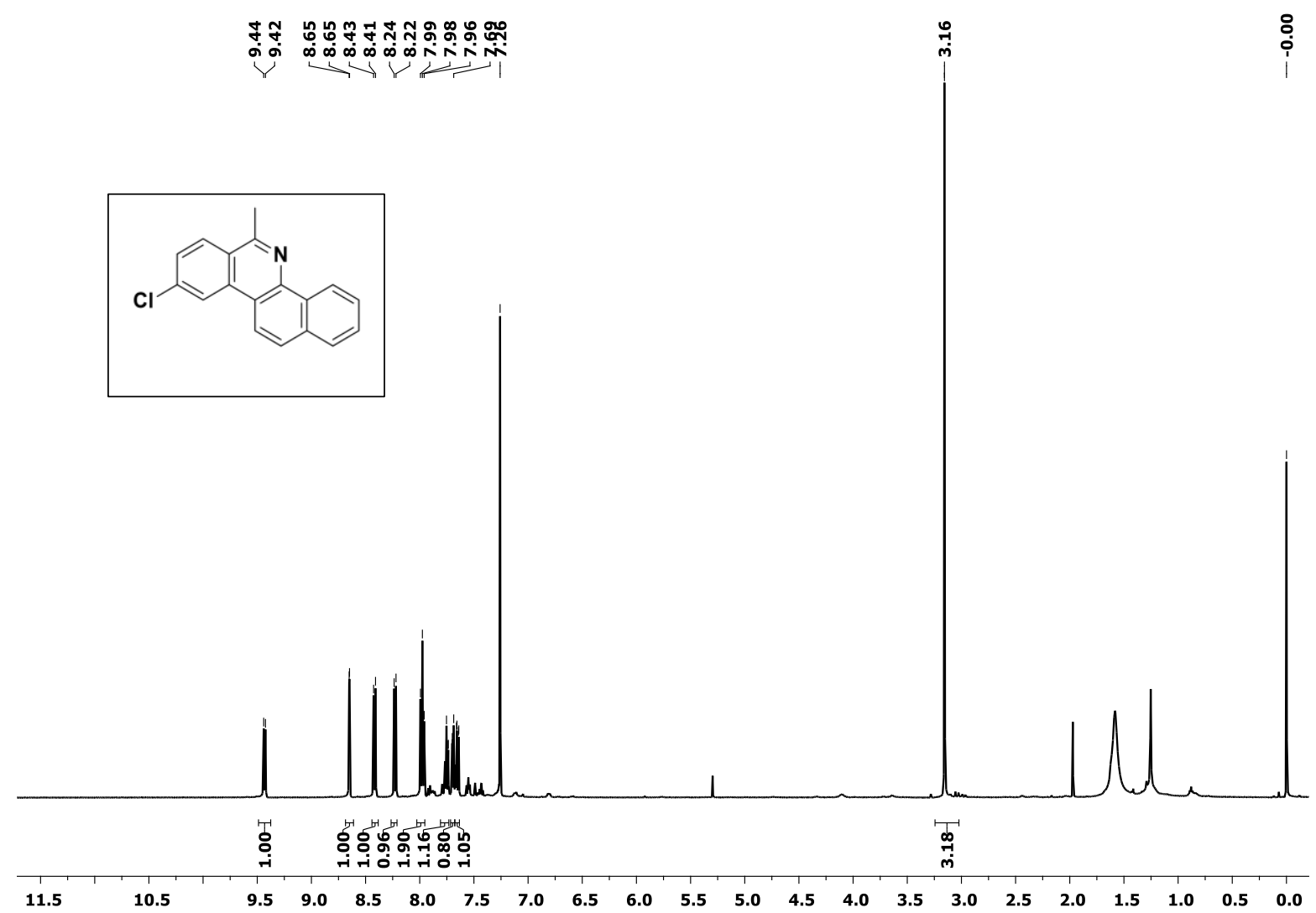

กุ.

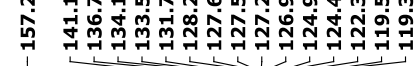

於

$\stackrel{\infty}{\infty}$
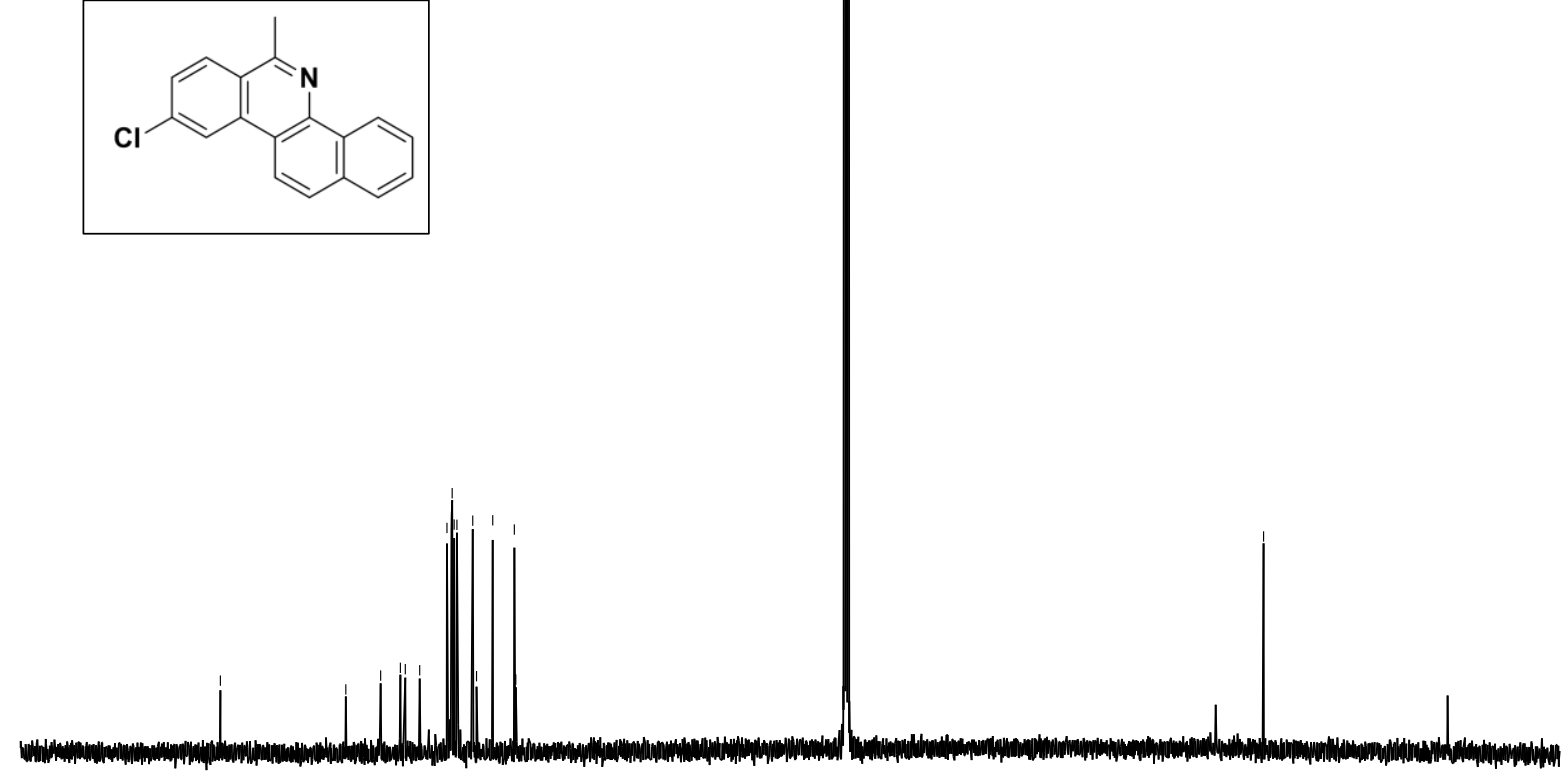
DEPT (135) NMR Spectrum of Compound 5a.

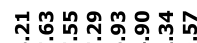

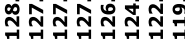

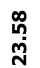

年<smiles>Cc1nc2c3ccccc3ccc2c2cc(Cl)ccc12</smiles>

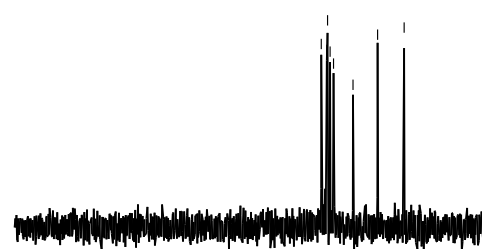

$\begin{array}{llllll}160 & 150 & 140 & 130 & 120 & 110\end{array}$

$\begin{array}{llc}100 & 90 & 80 \\ \mathrm{f1} & (\mathrm{ppm})\end{array}$

$\begin{array}{lllllll}60 & 50 & 40 & 30 & 20 & 10 & 0\end{array}$ 
${ }^{1} \mathrm{H}$ and ${ }^{13} \mathrm{C}$ NMR Spectra of Compound $\mathbf{5 b}$.

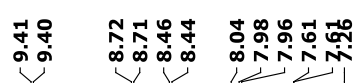

น̊ำ
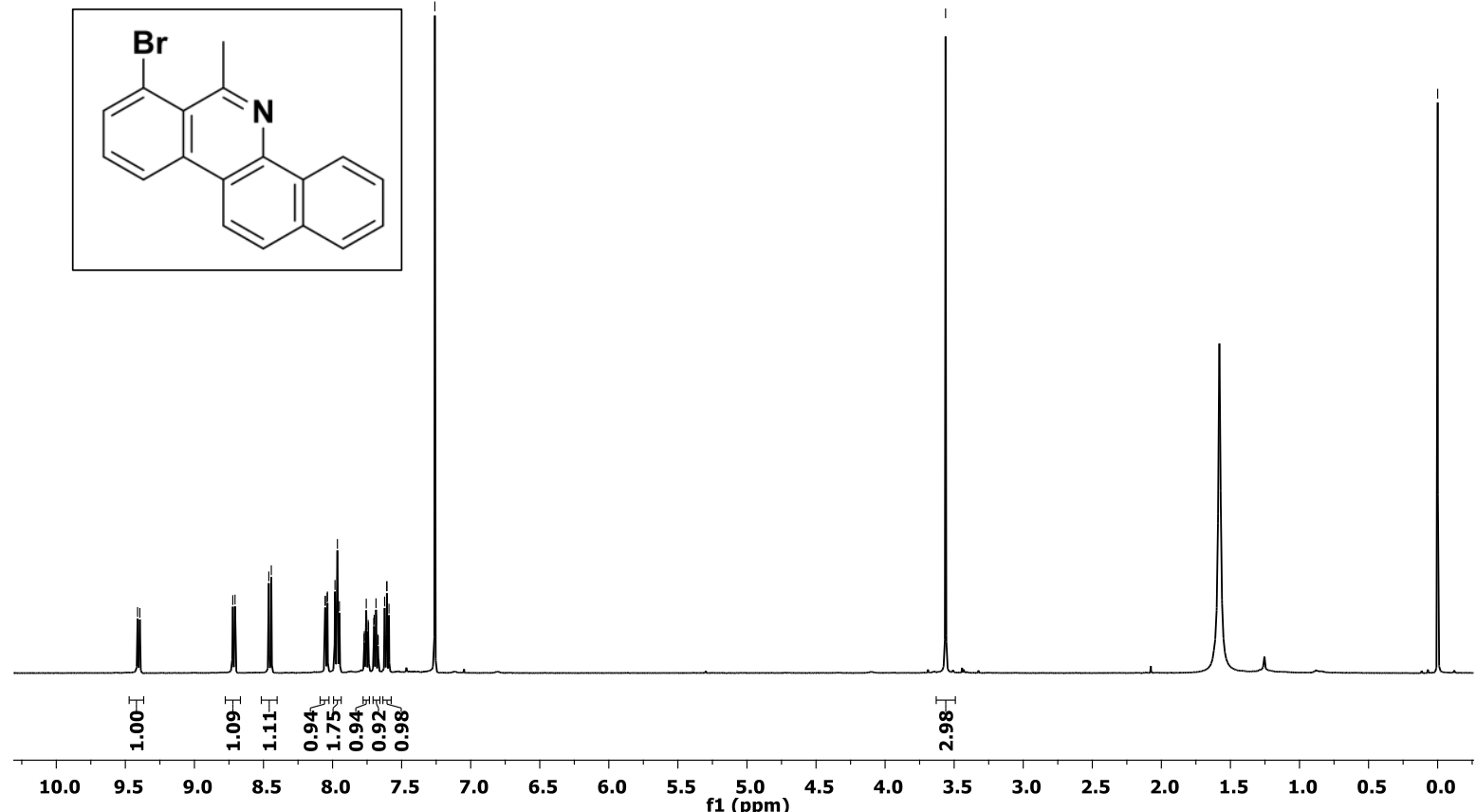

कి
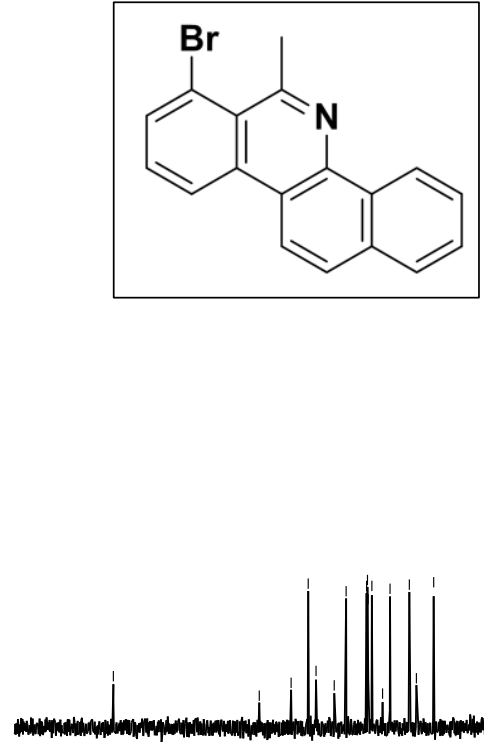
DEPT (135) NMR Spectrum of Compound $\mathbf{5 b}$.

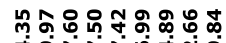

ํำ

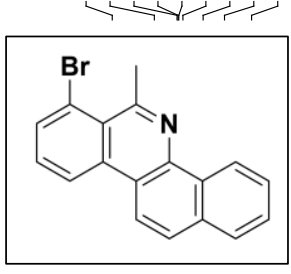

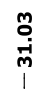

.

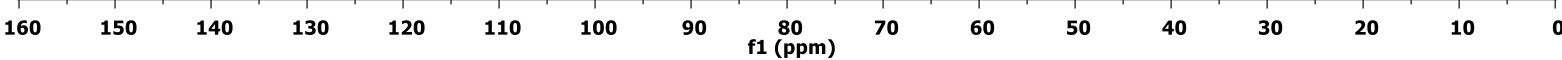

